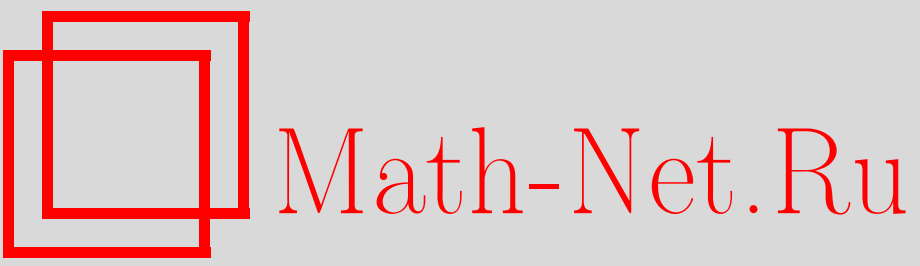

С. А. Назаров, Полиномиальное свойство самосопряженных эллиптических краевых задач и алгебраическое описание их атрибутов, УМН, 1999, том 54, выпуск $5,77-142$

DOI: https://doi.org/10.4213/rm204

Использование Общероссийского математического портала Math-Net.Ru подразумевает, что вы прочитали и согласны с пользовательским соглашением

http: //www . mathnet.ru/rus/agreement

Параметры загрузки:

IP : 54.198 .187 .58

26 апреля 2023 г., 11:04:33 


\title{
ПОЛИНОМИАЛЬНОЕ СВОЙСТВО САМОСОПРЯЖЕННЫХ ЭЛЛИПТИЧЕСКИХ КРАЕВЫХ ЗАДАЧ И АЛГЕБРАИЧЕСКОЕ ОПИСАНИЕ ИХ АТРИБУТОВ
}

\author{
C. А. НАЗАРОВ
}

Описан широкий класс краевых задач, для которых применение эллиптической теории сводится к элементарным алгебраическим операциям. Класс характеризуется полиномиальным свойством: полуторалинейная форма, отвечающая задаче, вырождается только на конечномерном линеале $\mathscr{P}$ векторных полиномов. Такое свойство обеспечивает эллиптичность краевой задачи, а ее ядро и коядро выражаются в терминах $\mathscr{P}$. Для областей с кусочно гладкими границами или выходами на бесконечность (коническими, цилиндрическими и периодическими) дополнительно предоставляются фрагменты асимптотических формул для решений и конкретизируются общие условные теоремы о фредгольмовости (в том числе, за счет модификации обычных весовых норм), а также вычисляется индекс оператора краевой задачи. Полиномиальное свойство помогает выполнить асимптотический анализ краевых задач в тонких областях и их сочленениях. Именно, несложные манипуляции с $\mathscr{P}$ дают возможность при редукции размерности предсказать размеры результирующей системы и порядки входящих в нее дифференциальных операторов, устанавливают ее эллиптичность и предоставляют полную информацию о строении пограничных слоев. Приведенные результаты иллюстрируются примерами из теории упругости и гидромеханики.

Библиографияя: 128 названий.

\section{СОДЕРЖАНИЕ}

Введение

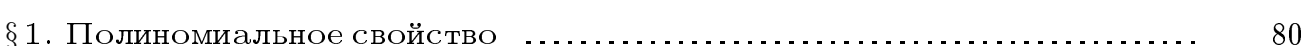

$\S 2$. Краевые задачи в областях с кусочно гладкой границей $\ldots . . . . . . . .992$

§3. Краевые задачи в областях с цилиндрическими выходами на беско-

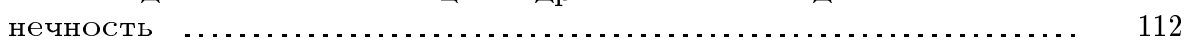

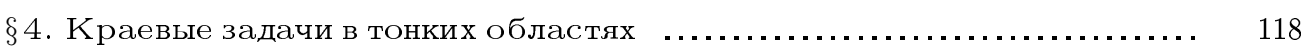

$\S 5$. Пограничные слои для тонких областей и их сочленений $\ldots . . \ldots \ldots . . .127$

Список литературы ..................................................... 137

\section{Введение}

Систематические исследования краевых задач для эллиптических систем уравнений в частных производных сформировали разветвленную теорию, стержневыми 
результатами которой являются: реализация краевых задач в областях с гладкими границами как фредгольмовых операторов в классах Гёльдера и $L_{p}([1]-[5]$ и др.), а также пространствах распределений ([5]-[8] и др.); вариационные постановки задач в гильбертовых энергетических пространствах ([9]-[12], [5] и др.); построение шкал пространств с весовыми нормами для краевых задач в областях с кусочно гладкими границами (угловые и конические точки, ребра, выходы на бесконечность и пр.) и асимптотические формулы для решений ([13]-[21] и др.). Эти и иные результаты, в частности, асимптотические методы, интенсивно разрабатываемые в последнее время, оказали сушественное влияние на развитие многих прикладных дисциплин и были востребованы в разнообразных задачах теории дифракции, механики деформируемых тел, гидродинамики, теории разрушения и пр. Цель данного обзора - описать широкий класс краевых задач, в основном, формально самосопряженных, для которых проверка свойств, необходимых для применения эллиптической теории, и конкретизация ее обших утверждений сводятся к простьм, чисто алгебраическим операциям (к ним в данном контексте причисляется и дифференцирование полиномов).

Начальным этапом, предваряющим использование аппарата эллиптической теории, служит изучение модельных задач. В случае областей с гладкими границами таковыми оказываются система уравнений с постоянными коэффициентами в $\mathbb{R}^{n}$ и аналогичная краевая задача в полупространстве $\mathbb{R}_{+}^{n}$. Если доказательство эллиптичности самой системы сводится к нахождению определителя матрищы, полиномиально зависяшей от параметра $\xi \in \mathbb{R}^{n}$, то для проверки условий накрытия этой системы граничными операторами (условий Шапиро-Лопатинского) требуется решить краевую задачу для системы обыкновенных дифференциальных уравнений на полуоси с параметром $\xi^{\prime} \in \mathbb{R}^{n-1}$. Во втором случае прямые выгисления становятся необозримыми уже в задаче Неймана для системы анизотропной теории упругости ( 21 свободная константа), а имеющееся описание условий накрытия в терминах корней неких полиномов ничуть не снижает их трудоемкости (ср. с комментарием к определению 10.1.1 в книге [7]). Еше сложнее обстоят дела с краевьми задачами в областях с ребрами, для которых возникают новые модельные задачи в двугранных углах и известные условия фредгольмовости оператора исходной задачи формулируются как требования однозначной разрешимости таких модельных задач в специальных весовых пространствах (см. [22]-[24], [20; гл. 6, 7] и др.). К сожалению, в общем случае пересказать эти требования в алгебраических терминах вряд ли возможно, а пример, в котором они заведомо не выполнены, доставляет все та же задача Неймана для трехмерного анизотропного упругого тела с гладким ребром на поверхности.

Для исправления ситуации с задачей Неймана в работах [25], [26] для гладких ребер и в [27] для многогранных углов были введены ступенчатые весовые пространства, обеспечивающие фредгольмовость оператора краевой задачи (распределение весовых множителей в соответствующих нормах отличается от использованного ранее в [28], [29] для других целей; см. далее п. $3 \S 2$ ). Попутно в [26] был намечен, а в [27] отчетливо выделен класс формально самосопряженных эллиптических систем с полиномиальным свойством - именно для них развитый подход приводил к успеху. Отметим, что в книге [19] задачи Неймана на полиэдральных областях изучались в пространствах Соболева-Слободецкого $H^{s}(\Omega)$ с нецельм (и не произвольным) показателем $s$.

Говоря приблизительно, полиномиальное свойство означает, что квадратичная (по- 
луторалинейная) форма, отвечаюшая краевой задаче, вырождается лишш на конечномерном линеале $\mathscr{P}$ векторных полиномов. По своей природе это свойство присуще всем стационарным задачам математической физики, в частности, упоминавшейся задаче анизотропной теории упругости. В [30] было замечено, что названное свойство гарантирует как эллиптичность системы, так и ее накрытие граничными операторами (в [26], [27] и [20], [31] эллиптичность краевых задач постулировалась). В [32] требования к структуре системы были значительно ослаблены при сохранении абсолютного большинства результатов (в терминологии из 11 ранее рассматривались матрицы дифференциальных операторов с простой структурой), а в [33] схема исследования была приспособлена к некоторым несамосопряженным краевым задачам (см. п. $5 \S 1$ и пример 1.13).

Если квадратичная форма $a$, порожденная эллиптическим по Петровскому оператором $\mathscr{L}$ порядка $2 m$, удовлетворяет "усиленному" неравенству Гординга

$$
\left\|\nabla^{m} u ; L_{2}(\Omega)\right\|^{2} \leqslant c a(u, u ; \Omega) \quad \forall u=\left(u_{1}, \ldots, u_{T}\right), \quad u_{j} \in H^{m}(\Omega),
$$

то $\mathscr{L}$, разумеется, обладает полиномиальньм свойством, причем линеал $\mathscr{P}$ состоит из всех полиномов степени не вьшше $m-1$. В [32] установлено, что полиномиальное свойство имеется и у формально положительных операторов [12], для которых неравенство типа Гординга-Корна

$$
\left\|u ; H^{m}(\Omega)\right\|^{2} \leqslant C\left(a(u, u ; \Omega)+\left\|u ; L_{2}(\Omega)\right\|^{2}\right) \quad \forall u \in H^{m}(\Omega)^{T}
$$

выводится в [12] также на основе чисто алгебраических предположений. Пример оператор-матрицы, обладающей полиномиальным свойством, но не являющейся формально положительной, доставляет система уравнений Стокса (см. далее п. $6 \S 1$ ).

В отличие от (0.1) неравенство (0.2) годится только для ограниченных областей, а в случае некомпактных границ требуются весовые неравенства Корна, в которых весовые множители определяются как дифференциальной структурой оператор-матрицы $\mathscr{L}$, так и геометрической формой выходов на бесконечность. Подобные неравенства известны лишь для весьма узкого класса операторов (см. [34]-[38] и др.), но сами краевые задачи в неограниченных областях возникают естественным образом при асимптотическом анализе различных сингулярных возмушений и, описьвая явление пограничного слоя, приобрели теперь немаловажное значение (см. [39]-[43] и др.). Поэтому далее обсуждаются задачи в областях с коническими, цилиндрическими и квазицилиндрическими (периодическими) выходами на бесконечность. Полиномиальное свойство не только помогает установить их фредгольмовость, но и позволяет выразить в терминах линеала $\mathscr{P}$ фрагменты асимптотических разложений решений, описать ядро и коядро оператора задачи, найти условия убывания решения при $|x| \rightarrow \infty$.

Основная идея большинства доказательств довольно-таки проста: вектор-функция $u$ конструируются так, чтобы при подстановке ее на обе позиции в формулу Грина часть слагаемых исчезала и возникало равенство $a(u, u ; \Omega)=0$, означающее, что $u \in \mathscr{P}$. Последнее включение используется либо для идентификации ядра оператора задачи, либо для отрицания предположенного свойства, если $u$ - заведомо не полином (центральньм моментом является конечномерность линеала $\mathscr{P}$ и в принципе он может состоять из произвольных, не обязательно полиномиальных функций, однако обобшения такого рода малосодержательны - см. замечание 3.1 в [32]). Ключевой 
трюк - подбор самих пробных функций $u$, и на этом пути удается получить частичную информацию о спектре пучков, порож денных модельными задачами в конусах, цилиндрах и квазицилиндрах. Более точно, конкретизируется строение канонических систем жордановых цепочек, отвечающих собственным числам пучков из некоторой узкой полосы на комплексной плоскости. Тем не менее, вкупе с обшими теоремами эллиптической теории (условия фредгольмовости оператора задачи, представления его коядра посредством ядра сопряженной задачи, формулы для прирашения индекса и т.п.) установленные отдельные свойства спектра дают возможность закончить исследование краевых задач и предоставить алгебраическое описание их атрибутов.

Применение полиномиального свойства в асимптотическом анализе не ограничивается исследованием пограничных слоев. В $\S 4$ изложены результаты [30] для краевых задач в тонких областях $\Omega(\varepsilon)$, которые при $\varepsilon \rightarrow+0$ стягиваются к подмногообразиям $S$ меньшей размерности (типичные примеры - пластины и стержни). Асимптотический подход подразумевает редукиию размерности - вывод результирующей задачи на $S$, из решений которой сооружается асимптотическое приближение к решению исходной задачи в $\Omega(\varepsilon)$. В [44], [45] (см. также [46; гл. 9] и [43; гл. 15, 16]) обнаружена аналогия в процедурах построения результируюших систем и жордановых цепочек родственных пучков. Как упоминалось в предыдущем абзаце, линеал полиномов $\mathscr{P}$ содержит ряд сведений о структуре жордановых цепочек, что позволило в [30] дать алгебраическое описание результируюшей задачи: при помоши несложных манипуляций с $\mathscr{P}$ устанавливаются размеры результируюшей системы и порядки входящих в нее дифференциальных операторов, проверяются априори ее эллиптичность и формальная самосопряженность. Подчеркнем, что в [30] изучены задачи теории осреднения (см. [47]-[51] и др.), однако для упрошения изложения в данном обзоре быстрые осциллящии коэффищиентов и гранищы области исключены из рассмотрения.

\section{§ 1. Полиномиальное свойство}

1. Матричные операторы простой структуры. Пусть $\mathscr{L}\left(x, D_{x}\right)$ есть $(T \times T)$ матрища, составленная из дифференциальных операторов

$$
\mathscr{L}_{j k}\left(x, \nabla_{x}\right)=\sum_{|\alpha| \leqslant t_{j}+t_{k}} l_{j k}^{\alpha}(x) \nabla_{x}^{\alpha}
$$

где $l_{j k}^{\alpha} \in C^{\infty}\left(\mathbb{R}^{n}\right), \alpha=\left(\alpha_{1}, \ldots, \alpha_{n}\right)$ - мультииндекс, $x=\left(x_{1}, \ldots, x_{n}\right)$ - точка в $\mathbb{R}^{n}$; $t_{1}, \ldots, t_{T}$ и $T$ - натуральные числа, $\nabla_{x}=\operatorname{grad}$,

$$
\nabla_{x}^{\alpha}=\partial^{|\alpha|} / \partial x_{1}^{\alpha_{1}} \cdots \partial x_{n}^{\alpha_{n}}, \quad|\alpha|=\alpha_{1}+\cdots+\alpha_{n}
$$

Предположим, что в любой области $\Omega \subset \mathbb{R}^{n}$ c $(n-1)$-мерной гладкой границей $\partial \Omega$ и компактным замыканием $\bar{\Omega}$ верна формула Грина

$$
(\mathscr{L} u, v)_{\Omega}+(\mathscr{N} u, \mathscr{D} v)_{\partial \Omega}=a(u, v ; \Omega)
$$

где $u=\left(u_{1}, \ldots, u_{T}\right), v=\left(v_{1}, \ldots, v_{T}\right) \in C^{\infty}(\bar{\Omega})^{T},(\cdot, \cdot) \Xi-$ скалярное произведение в пространстве $L_{2}(\Xi)$, скалярном или векторном; $\mathscr{N}$ и $\mathscr{D}-(m \times T)$-матрищы, 
$m=t_{1}+\cdots+t_{T} ; \mathscr{D}$ - система Дирихле (см. [5], [6], [8] и др.), $\mathscr{N}\left(x, \nabla_{x}\right)-$ оператор краевых условий Неймана; $a$ - симметричная полуторалинейная форма

$$
a(u, v ; \Xi)=\sum_{j, k=1}^{T} \sum_{|\sigma| \leqslant t_{j}} \sum_{|\gamma| \leqslant t_{k}} \int_{\Xi} a_{j k}^{\sigma \gamma}(x) \nabla_{x}^{\gamma} u_{k} \nabla_{x}^{\sigma} \overline{v_{j}} d x,
$$

причем $a_{j k}^{\sigma \gamma} \in C^{\infty}\left(\mathbb{R}^{n}\right)$ и согласно сказанному

$$
a(u, v ; \Xi)=\overline{a(v, u ; \Xi)} .
$$

ЗАмечание 1.1. Далее используется лишь такое свойство системы Дирихле:

$$
\forall \Phi \in C^{\infty}(\partial \Omega)^{m} \quad \exists U \in C^{\infty}(\bar{\Omega})^{T}: \quad \mathscr{D} U=\Phi \text { на } \partial \Omega .
$$

Образуем $(m \times T)$-матрицы $\mathscr{B}$ и $\mathscr{T}$ из строк $\mathscr{B}_{q}$ и $\mathscr{T}_{q}$, выбранных одним из следующих двух способов:

$$
\mathscr{B}_{q}=\mathscr{N}_{q}, \mathscr{T}_{q}=\mathscr{D}_{q} \text { или } \mathscr{B}_{q}=\mathscr{D}_{q}, \mathscr{T}_{q}=-\mathscr{N}_{q}
$$

(своим для каждого $q=1, \ldots, m)$. Из $(1.2)$ и $(1.4),(1.6)$ вытекает "полная" формула Грина

$$
(\mathscr{L} u, v)_{\Omega}+(\mathscr{B} u, \mathscr{T} v)_{\partial \Omega}=(u, \mathscr{L} v)_{\Omega}+(\mathscr{T} u, \mathscr{B} v)_{\partial \Omega},
$$

устанавливающая формальную самосопряженность краевой задачи

$$
\begin{aligned}
& \mathscr{L}\left(x, \nabla_{x}\right) u(x)=f(x), \quad x \in \Omega, \\
& \mathscr{B}\left(x, \nabla_{x}\right) u(x)=g(x), \quad x \in \partial \Omega,
\end{aligned}
$$

в которой $f=\left(f_{1}, \ldots, f_{T}\right)$ и $g=\left(g_{1}, \ldots, g_{m}\right)$. Подчеркнем, что граничные операторы $\mathscr{D}, \mathscr{B}$ и т. п. зависят от области $\Omega$, но это в обозначениях не указывается.

Один из классов оператор-матриц простой структуры возникает при использовании матричной записи. Именно, векторы $u$ и $f, g$ интерпретируются как столбцы, а в формулах

$$
\begin{gathered}
\mathscr{L}\left(x, \nabla_{x}\right)=\Lambda\left(x, \nabla_{x}\right)^{*} A(x) \Lambda\left(x, \nabla_{x}\right), \\
a(u, v ; \Xi)=(A \Lambda u, \Lambda v)_{\Xi}
\end{gathered}
$$

под $\Lambda$ и $A$ подразумеваются матрицы размера $N \times T$ и $N \times N$ соответственно $(N-$ некоторое натуральное число). Элементы $\Lambda_{h j}\left(x, \nabla_{x}\right)$ матрицы $\Lambda$ - дифференциальные операторы порядка $t_{j}$ с гладкими коэффициентами, а $A$ - матрица-функция, симметрическая, положительно определенная и бесконечно дифференцируемая во всех точках $x \in \mathbb{R}^{n}$. Кроме того, $\Lambda$ и $\Lambda^{*}$ - формально сопряженные операторы. Нетрудно убедиться в справедливости представлений $(1.1),(1.3)$ и неравенства

$$
a(u, u ; \Omega) \geqslant c_{A}(\Omega)\left\|\Lambda u ; L_{2}(\Omega)\right\|^{2},
$$

где $c_{A}(\Omega)$ - положительная постоянная, зависящая от $A$ и $\Omega$. 
В результате интегрирования по частям (в том числе и на границе $\partial \Omega$ ) появляется формула Грина (1.2). Так, при $t_{1}=\cdots=t_{T}=1$ имеем $m=T$ и

$$
\mathscr{D} v=v, \quad \mathscr{N}\left(x, \nabla_{x}\right) u=\Lambda(\nu(x))^{*} A(x) \Lambda\left(x, \nabla_{x}\right),
$$

где $\nu$ - единичньй вектор внешней нормали к $\partial \Omega$. Ясно, что всегда дело можно свести к ситуации, в которой $A$ - единичная матрица, но запись $(1.10),(1.11)$ более удобна для операторов с постоянными коэффициентами. В частности, $\Lambda\left(\nabla_{x}\right)^{*}=\Lambda\left(-\nabla_{x}\right)^{t}$ в случае вешественных коэффициентов у $\Lambda ; t$ - знак транспонирования.

2. Матричные операторы усложненной структуры. Расширим матрищу $\mathscr{L}$ до размера $T^{\prime} \times T^{\prime}$ :

$$
\mathscr{L}^{\prime}\left(x, \nabla_{x}\right)=\left(\begin{array}{cc}
\mathscr{L}\left(x, \nabla_{x}\right), & \mathscr{Q}^{*}\left(x, \nabla_{x}\right) \\
\mathscr{Q}\left(x, \nabla_{x}\right), & 0
\end{array}\right)
$$

Здесь $T^{\prime}>T, \mathscr{Q}$ - матрица размера $\left(T^{\prime}-T\right) \times T$ с элементами $\mathscr{Q}_{p k}$, причем ord $\mathscr{Q}_{p k}=$ $t_{p}+t_{k}, p=T+1, \ldots, T^{\prime}, k=1, \ldots$, и и

$$
t_{T+1}, \ldots, t_{T^{\prime}} \leqslant 0 .
$$

Ясно, что $m^{\prime}=t_{1}+\cdots+t_{T^{\prime}} \leqslant m$.

Оператор (1.14) наследует формальную самосопряженность от $\mathscr{L}$. Опишем краевые условия, которыми можно снабдить $\mathscr{L}^{\prime}$. Пусть на $\partial \Omega$ вьполняются представления

$$
\mathscr{N}=\left(\begin{array}{c}
N^{\prime} \\
\mathcal{N}^{\prime \prime}
\end{array}\right), \quad \mathscr{D}=\left(\begin{array}{c}
\mathscr{D}^{\prime} \\
\mathscr{S} \mathscr{Q}+\mathscr{M} \mathscr{D}^{\prime}
\end{array}\right)
$$

и одновременно верна формула Грина

$$
\left(\mathscr{Q}^{*} u^{\prime}, v\right)_{\Omega}=\left(u^{\prime}, \mathscr{Q} v\right)_{\Omega}-\left(\mathscr{K} u^{\prime}, \mathscr{D}^{\prime} v\right)_{\partial \Omega}
$$

для любых гладких функций $u^{\prime}=\left(u_{T+1}, \ldots, u_{T}\right)$ и $v=\left(v_{1}, \ldots, v_{T}\right)$. Матрицы $\mathscr{K}, \mathscr{S}$ и $\mathscr{N}^{\prime}, \mathscr{D}^{\prime}$ обладают размерами $m \times\left(T^{\prime}-T\right),\left(m-m^{\prime}\right) \times\left(T^{\prime}-T\right)$ и $m^{\prime} \times T$ соответственно, а их элементы - дифференциальные операторы на $\partial \Omega$. Наконец, $\mathscr{M}\left(s, \nabla_{s}\right)-$ оператор-матрица размером $\left(m-m^{\prime}\right) \times m$, а через $s$, несколько вольно, обозначаются координаты точки в карте подходящего атласа на $(n-1)$-мерном подмногообразии $\partial \Omega$.

ЗАмечАниЕ 1.2 . Пусть $d_{q}+t_{j}$ и $n_{q}+t_{k}-$ порядки дифференциальных операторов $\mathscr{D}_{q j}$ и $\mathscr{N}_{q k}$. В соответствии с (1.2) имеем

$$
t_{j}+t_{k}-1=-1+\operatorname{ord} \mathscr{L}_{j k}=\operatorname{ord} \mathscr{D}_{q j}+\operatorname{ord} \mathscr{N}_{q k}=t_{j}+t_{k}+d_{q}+n_{q},
$$

т.е. $n_{q}=-1-d_{k} \geqslant 0$ и $d_{k}<0$. Таким образом, требование $(1.15)$, приводящее к неравенству ord $\mathscr{Q}_{p j} \leqslant t_{j}$, необходимо для принципиальной возможности подставить в (1.17) подматрицу $\mathscr{D}^{\prime}$ из (1.16).

Матрицы $\mathscr{B}^{\prime}$ и $\mathscr{T}^{\prime}$ соорудим из строк $\mathscr{B}_{q}^{\prime}$ и $\mathscr{T}_{q}^{\prime}, q=1, \ldots, m^{\prime}$, определяемых, как и в (1.6), одним из двух способов

$$
\begin{aligned}
& \mathscr{B}_{q}^{\prime}=\left(\mathscr{N}^{\prime}+\mathscr{M}^{*} \mathscr{N}^{\prime \prime}, \mathscr{K}\right)_{q}, \quad \mathscr{T}_{q}^{\prime}=\left(\mathscr{D}^{\prime}, 0\right)_{q} \\
& \text { или } \\
& \mathscr{B}_{q}^{\prime}=\left(\mathscr{D}^{\prime}, 0\right)_{q}, \quad \mathscr{T}_{q}=-\left(\mathscr{N}^{\prime}+\mathscr{M}^{*} \mathscr{N}^{\prime \prime}, \mathscr{K}\right)_{q} .
\end{aligned}
$$

В (1.18) фигурируют строки составных $\left(m^{\prime} \times \mathscr{T}^{\prime}\right)$-матриц; в частности, к $\left(m^{\prime} \times T\right)$-матрище $\mathscr{D}^{\prime}$ справа приписаны $T^{\prime}-T$ нулевых $m^{\prime}$-столбцов. 
ЛЕмма 1.3 ([32]). 1) Справедлива формула Грина

$$
\begin{aligned}
\left(\mathscr{L}^{\prime} \mathbf{u}, \mathbf{v}\right)_{\Omega}+\left(\mathscr{B}^{\prime} \mathbf{u}, \mathscr{T}^{\prime} \mathbf{v}\right)_{\partial \Omega}-\left(\mathscr{S} \mathscr{Q} u, \mathscr{N}^{\prime \prime} v\right)_{\partial \Omega} \\
=\left(\mathbf{u}, \mathscr{L}^{\prime} \mathbf{v}\right)_{\Omega}+\left(\mathscr{T}^{\prime} \mathbf{u}, \mathscr{B}^{\prime} \mathbf{v}\right)_{\partial \Omega}-\left(\mathscr{N}^{\prime \prime} u, \mathscr{S} \mathscr{Q} v\right)_{\partial \Omega}
\end{aligned}
$$

в которой $\mathbf{u}=\left(u, u^{\prime}\right)=\left(u_{1}, \ldots, u_{T^{\prime}}\right)$ u $\mathbf{v}=\left(v, v^{\prime}\right)-$ произвольные функции из $C^{\infty}(\bar{\Omega})^{T^{\prime}}$.

2) $E c \curvearrowright u \mathbf{u}, \mathbf{v} \in C^{\infty}(\bar{\Omega})^{T^{\prime}}$ u $\mathscr{Q} u=\mathscr{Q} v=0$ на $\partial \Omega, m o$

$$
(\mathscr{L} \mathbf{u}, \mathbf{v})_{\Omega}+\left(\left[\mathscr{N}^{\prime}+\mathscr{M}^{*} \mathscr{N}^{\prime \prime}\right] u+\mathscr{K} u^{\prime}, \mathscr{D}^{\prime} v\right)_{\partial \Omega}=a(u, v ; \Omega)
$$

Подчеркнем, что в (1.19) фигурируют новые (по сравнению с (1.7)) интегралы по поверхности $\partial \Omega$, которые исчезают при выполнении условий леммы $1.3(2)$. Далее рассмотрение краевой задачи $(1.8),(1.9)$ и аналогичной ей задачи $(1.8)^{\prime},(1.9)^{\prime}$ с операторами $\mathscr{L}^{\prime}, \mathscr{B}^{\prime}$ ведется параллельно, причем (1.19) и (1.20) придаются роли формул Грина (1.7) и (1.2). (Так, например, задача $(1.8)^{\prime},(1.9)^{\prime}$ самосопряжена относительно формулы (1.19).) Для упрошения формулировок в следуюших разделах, если не оговорено противное, под (1.8), (1.9) подразумеваем обе упомянутые задачи, а под $\mathscr{L}$ - как оператор простой структуры, так и оператор (1.14) сложной. В связи с этим позволяем себе вместо $\mathbf{u}, T^{\prime}$ и т. п. писать $u, T$ и т.п.

3. Полиномиальное свойство. Будем говорить, что пара $\{\mathscr{L}, a\}$ обладает noлиномиальныцм свойством, если для любой (в том числе неограниченной) области $\Xi \subset \mathbb{R}^{n}$ верно следуюшее утверждение:

$1^{0} . u \in C^{\infty}(\bar{\Xi})^{T}, \mathscr{L} u=0$ в $\Xi, a(u, u ; \Xi)=\left.0 \Leftrightarrow u \in \mathscr{P}\right|_{\Omega}$.

Здесь фигурирует некоторый конечномерный линеал полиномов переменных $x \mathrm{c}$ коэффишиентами из $\mathbb{C}^{T}$.

При условии

$$
2^{0} . u \in C^{\infty}(\bar{\Xi})^{T}, a(u, u ; \Xi)=\left.0 \Leftrightarrow u \in \mathscr{P}^{a}\right|_{\Xi}
$$

полиномиальное свойство присуще самой форме (а не паре "оператор-форма"). Конечномерный линеал $\mathscr{P}^{a}$, разумеется, может отличаться от $\mathscr{P}$ (и не только в случае $\left.T^{\prime}>T\right)$.

ЗАмЕчаниЕ 1.4. Для операторов усложненной структуры начало строки $1^{0}$ читается так: $\mathbf{u} \in C^{\infty}(\bar{\Xi})^{T^{\prime}}, \mathscr{L}^{\prime} \mathbf{u}=0 \ldots$ Строка $2^{0}$ в изменениях не нуждается - речь в ней идет о фрагменте $u$ функции $\mathbf{u}=\left(u, u^{\prime}\right)$.

Если $\mathscr{L}$ - оператор простой структуры, то $\mathscr{P}=\{p \in \mathscr{P} a: \mathscr{L} p=0\}$, а полиномиальное свойство имеется у формы $а$ в полном объеме тогда, когда вьполнено следуюшее требование:

$3^{0} . a(p, v ; \Xi)=0 \forall p \in \mathscr{P}, v \in C^{\infty}(\bar{\Xi})^{T}$.

ПрЕДЛОЖЕНИЕ 1.5 ([32]). Для операторов простой структуры условие $1^{0}$ вытекает из $2^{0}$, а в случае области $\Omega=\Xi$ слладкой границей требование $3^{0}$ равносильно такому:

$$
4^{0} . \mathscr{N}\left(x, \nabla_{x}\right) p(x)=0, x \in \partial \Omega \quad \forall p \in \mathscr{P} \text {. }
$$

Отметим, что в доказательстве используется свойство (1.5) системы Дирихле и в формулу Грина (1.2) подставляются функции $u=p$ и $v=U$. 
4. Формально положительные операторы ([12]). Оператор-матрица $\Lambda\left(x, \nabla_{x}\right)$ назьвается алгебраически комплектной, а оператор (1.10) - формально положительным, если при некотором натуральном $\rho_{\Lambda}$ вьполнено следующее условие:

$5^{0}$. для всякой строки полиномов $P=\left(P_{1}, \ldots, P_{T}\right)$, обладающей обобщенной однородностью с показателем $\rho \geqslant \rho_{\Lambda}$,

$$
P_{k}(z \xi)=z^{t_{k}+\rho} P_{k}(\xi) \quad \forall z \in \mathbb{R}, \quad \xi \in \mathbb{R}^{n}, \quad k=1, \ldots, T
$$

найдется строка дифференииальных операторов $Q=\left(Q_{1}, \ldots, Q_{N}\right)$, такая, что

$$
P\left(\nabla_{x}\right)=Q\left(x, \nabla_{x}\right) \Lambda\left(x, \nabla_{x}\right)
$$

Tак как ord $\Lambda_{h k}=t_{k}$, в силу (1.21) и (1.22) ord $Q_{h}=\rho$. Если коэффициенты операторов постоянны, то равенство (1.22) переписывается в виде $P(\xi)=Q(\xi) \Lambda(\xi), \xi \in \mathbb{R}^{n}$, объясняющем происхождение термина "алгебраическая комплектность".

ПРЕДЛОЖЕНИЕ 1.6 ([32]). При условии $5^{0}$ оператор $\mathscr{L}$ и форма а из (1.10) и (1.11) удовлетворяют $1^{0}-4^{0}$, причем

$$
\mathscr{P}=\mathscr{P}^{a}=\left\{p: \Lambda\left(x, \nabla_{x}\right) p(x)=0, x \in \Xi\right\}
$$

Понятия, введенные в $5^{0}$, находят применение и для операторов усложненной структуры.

ПРЕДЛОЖЕНИЕ 1.7 ([32]). Пусть матрица $\mathscr{Q}^{*}\left(x, \nabla_{x}\right)$ алгебраически комплектна и форма а из (1.3) подчинена условию $2^{0}$, причем $\mathscr{L} p=0$ для всех $p \in \mathscr{P}$, таких, что $\mathscr{Q} p=0$. Тогда пара $\left\{\mathscr{L}^{\prime}, a\right\}$ обладает полиномиальным свойством $1^{0}$.

5. Эллиптичность краевой задачи. Обозначим через $\mathscr{L}^{0}$ и $a^{0}$ оператор и форму, получаюшиеся из $\mathscr{L}$ и а замораживанием коэффициентов в точке $x^{0} \in \mathbb{R}^{n}$, т.е. в согласии с (1.1)

$$
\mathscr{L}^{0}=\left(\mathscr{L}_{j k}^{0}\right)_{j, k=1}^{T}, \quad \mathscr{L}_{j k}^{0}\left(x, \nabla_{x}\right)=\sum_{|\alpha|=t_{j}+t_{k}} l_{j k}^{\alpha}\left(x^{0}\right) \nabla_{x}^{\alpha},
$$

а выражение $a^{0}(u, v ; \Xi)$ получается из $(1.3)$ заменой $a_{j k}^{\sigma \gamma}(x)$ на $a_{j k}^{\sigma \gamma}\left(x^{0}\right)$ и сужением суммирования до $|\sigma|=t_{j},|\gamma|=t_{k}$. Точно так же определяются главные части $\mathscr{B}^{0}$, $\mathscr{Q}^{0}, \mathscr{L}^{\prime 0}$ и т. п. операторов $\mathscr{B}, \mathscr{Q}, \mathscr{L}^{\prime}$ и т. п. Элементам $\mathscr{B} q j$ матрищы $\mathscr{B}$ приписываются порядки $r_{q}+t_{j}$.

Будем говорить, что полиномиальное свойство у пары $\{\mathscr{L}, a\}$ наследуется $n p u$ замораживании в точке $x^{0}$, если для $\left\{\mathscr{L}^{0}, a^{0}\right\}$ справедливо требование $1^{0}$ (быть может, с новым линеалом полиномов; см. далее пример 1.16). Такая же терминология используется для $2^{0}$ и $3^{0}$. 
ЗАМЕчАниЕ 1.8 . Пусть алгебраически комплектная матрица $\Lambda\left(\nabla_{x}\right)$ составлена из однородных дифференциальных операторов с постоянньми коэффициентами. Тогда полиномиальное свойство пары $\{\mathscr{L}, a\}$ из (1.10), (1.11) выдерживает замораживание коэффициентов в любой точке.

В очередных утверждениях устанавливается, что полиномиальное свойство обеспечивает әллиптичность краевой задачи (1.8), (1.9) и, как следствие, фредгольмовость ее оператора

$$
\mathscr{A}^{l}=\{\mathscr{L}, \mathscr{B}\}: \mathscr{D}^{l} H(\Omega) \rightarrow \mathscr{R}^{l} H(\Omega) ;
$$

здесь $H^{s}(\Omega)$ и $H^{s+1 / 2}(\partial \Omega)$ - пространства Соболева-Слободецкого,

$$
\begin{aligned}
& \mathscr{D}^{l} H(\Omega)=\prod_{k=1}^{T} H^{l+t_{k}}(\Omega), \\
& \mathscr{R}^{l} H(\Omega)=\prod_{j=1}^{T} H^{l-t_{j}}(\Omega) \times \prod_{q=1}^{m} H^{l-r_{q}-1 / 2}(\partial \Omega) .
\end{aligned}
$$

Подчеркнем, что при постановке самой краевой задачи не требовалось ничего, кроме правильной структуры оператора $\mathscr{L}$ (см. (1.1) или (1.14)) и наличия формул Грина $(1.2)$ и (1.17), приводящих к (1.7) и (1.19). Навязанная структура гарантирует лишь непрерывность отображения (1.25) при условии

$$
l \geqslant \max \left\{t_{1}, \ldots, t_{T} ; 1+r_{1}, \ldots, 1+r_{m}\right\}=\max \left\{t_{1}, \ldots, t_{T}\right\} \equiv \max \mathbf{t} .
$$

Tеорема 1.9 ([30], [32]). 1) Если для любой точки $x^{0} \in \bar{\Omega}$ пара $\left\{\mathscr{L}^{0}, a^{0}\right\}$ обладает полиномиальныцм свойством, то задача (1.8), (1.9) әллиптическая и оператор $\mathscr{A l}^{l}$ из (1.25), (1.28) фредгольмов.

2) Если полиномиальное свойство пары $\{\mathscr{L}, a\}$ въдерживает замораживание во всех точках $x^{0} \in \bar{\Omega}$, mо

$$
\begin{aligned}
\operatorname{ker} \mathscr{A}^{l} & =\mathscr{P}(\mathscr{B}) \equiv\{p \in \mathscr{P}: \mathscr{B} p=0 \text { на } \partial \Omega\}, \\
\operatorname{coker} \mathscr{A}^{l} & =\left\{\left(p, \mathscr{T} p, \mathscr{N}^{\prime} p^{\prime}\right): p \in \mathscr{P}(\mathscr{B})\right\},
\end{aligned}
$$

но для операторов простой структуры компонента $\mathscr{N}^{\prime} p^{\prime}$ убирается.

Итак, формула (1.29) по линеалу полиномов $\mathscr{P}$ выстраивает все решения однородной задачи $(1.8),(1.9)$. Кроме того, в соответствии с (1.30) условия ее разрешимости принимают вид

$$
(f, p)_{\Omega}+(g, \mathscr{T} p)_{\partial \Omega}=0 \quad \forall p \in \mathscr{P}(\mathscr{B})
$$

для операторов простой структуры и вид

$$
(f, p)_{\Omega}+(g, \mathscr{T} p)_{\partial \Omega}+\left(\mathscr{S} f^{\prime}, \mathscr{N}^{\prime \prime} p\right)_{\partial \Omega}=0 \quad \forall p \in \mathscr{P}(\mathscr{B})
$$

в случае усложнения структуры. Проблем с определением последнего интеграла в правой части $(1.31)^{\prime}$ не возникает. В самом деле,

$$
\begin{gathered}
f^{\prime}=\left(f_{1+T^{\prime}}, \ldots, f_{T}\right) \in H^{l-t_{1+T}}(\Omega) \times \cdots \times H^{l-t_{T^{\prime}}}(\Omega) \subset H^{l}(\Omega)^{T^{\prime}-T}, \\
\mathscr{S} f^{\prime} \in H^{l-d_{1+m^{\prime}}-1 / 2}(\partial \Omega) \times \cdots \times H^{l-d_{m}-1 / 2}(\partial \Omega) \subset H^{l+1 / 2}(\partial \Omega)^{m-m^{\prime}},
\end{gathered}
$$

так как в силу требования (1.15), представления (1.16) и замечания 1.2

$$
\begin{gathered}
\operatorname{ord} \mathscr{S}_{q p}=\operatorname{ord} \mathscr{D}_{q j}-\operatorname{ord} \mathscr{Q}_{p j}=d_{p}+t_{j}-\left(t_{p}+t_{j}\right)=d_{p}-t_{p}<-t_{p}, \\
q=1+m^{\prime}, \ldots, m, \quad p=1+T, \ldots, T^{\prime}, \quad j=1, \ldots, T .
\end{gathered}
$$


ЗАМЕЧАнИЕ 1.10. Формулой Грина (1.7), приводящей к обычным условиям разрешимости (1.31), могут обладать не только операторы простой структуры (см. далее пример 1.16).

Приведем пояснения к доказательству теоремы 1.9. Равенство (1.29) выводится путем обращения к полиномиальному свойству $1^{0}$ и подстановки произвольного решения однородной задачи (1.8), (1.9) в формулу Грина (1.2) или (1.20), левая часть которой исчезает. Аналогично равенство (1.30) получается при рассмотрении сопряженной задачи, совпадаюшей с (1.8), (1.9), причем переход от сопряженного оператора $\left(\mathscr{A}^{l}\right)^{*}$, заданного на пространстве распределений, к оператору $\mathscr{A}^{l}$ осушествляется за счет результатов [5], [6], [8] в случае (1.7) и [52] в случае (1.20). Продемонстрируем теперь, как делается заключение об эллиптичности, - используемьй прием [30] является частью доказательств многих утверждений в обзоре.

Отсутствие эллиптичности у матрицы $\mathscr{L}^{0}\left(\nabla_{x}\right)$ означает, что $\operatorname{det} \mathscr{L}^{0}(\theta)=0$ в некоторой точке $\theta$ единичной сферы $\mathbb{S}^{n-1}$ и, следовательно, существует такой столбец $b=\left(b_{1}, \ldots, b_{T}\right)^{t}$, что $\mathscr{L}^{0}(\theta) b=0 \in \mathbb{C}^{T}$ и $|b| \neq 0$. Не ограничивая обшности, считаем, что $\theta=e^{1}$, где $e^{h}-$ орт оси $x_{h}$. Положим

$$
w=\left(w_{1}, \ldots, w_{T}\right), \quad w_{j}(x)=b_{j} \frac{d^{s-t_{j}} W}{d x_{n}^{s-t_{j}}}\left(x_{n}\right), \quad j=1, \ldots, T,
$$

где $W \in C_{0}^{\infty}(0,1)$ и $s$ - натуральное число, $s \geqslant \max \left\{t_{1}, \ldots, t_{T}\right\}$. Очевидно, что $\mathscr{L}^{0}\left(\nabla_{x}\right) w=0$ и, в частности, $\mathscr{Q}^{0}\left(\nabla_{x}\right) w=0$ для оператора из (1.14). Таким образом, можно применить формулу Грина (1.2) на кубе $\mathbb{Q}=\left\{x: 0<x_{p}<1\right\}$. Подставив в эту формулу на обе позиции построенную функцию $w$, убеждаемся в том, что $a(w, w ; \mathbb{Q})=0$, поскольку поверхностные интегралы по противоположным граням либо взаимно сокрашаются из-за разнонаправленности нормалей, либо аннулируются благодаря множителю $W \in C^{\infty}(0,1)$. Итак, все требования из посылки в $1^{0}$ вьполнены, т.е. $w$ - полином, что возможно лишш при $b=0$ ввиду произвольности $W$. Найденное противоречие доказьвает эллиптичность $\mathscr{L}^{0}\left(\nabla_{x}\right)$. Лишь незначительные модификации конструкции (1.32) нужны для проверки условий накрытия $\mathscr{L}^{0}\left(\nabla_{x}\right)$ граничным оператором $\mathscr{B}^{0}\left(\nabla_{x}\right)$ (см. [30], [32]).

Приведенные рассуждения опираются на формулу Грина (1.2) в области с кусочно гладкой границей (в кубе), в то время как она (формула) постулировалась только при гладкой поверхности $\partial \Omega$. Эта неувязка устраняется при помоши стандартного трюка. В формулу Грина на более широкой области подставляется произведение

$$
w^{\delta}(x)=\chi_{\delta}\left(x_{1}\right) \times \cdots \times \chi_{\delta}\left(x_{n}\right) w(x),
$$

где $\chi_{0}$ - характеристическая функция отрезка $[0,1]$, а $t \mapsto \chi_{\delta}(t)$ - та же "ступенька", сглаженная в $\delta$-окрестностях точек $t=0$ и $t=1$. Предельный переход $\delta \rightarrow+0$ превращает объемные интегралы, содержащие производные множителей $\chi_{\delta}\left(x_{h}\right)$, в поверхностные и обеспечивает нужную формулу Грина на $\mathbb{Q}$, отличаюшуюся, вообше говоря, от (1.2) строением интегралов по границе, но вполне заменяющую (1.2).

Отметим, что для неотрищательной формы $a$ (см., например, (1.12)) из равенства $a(u, u ; \Omega)=0$ вытекает, что $a(u, u ; \omega)=0$ при любой подобласти $\omega \subset \Omega$, а значит, в $1^{0}-3^{0}$ достаточно брать области $\Xi$ с компактным замыканием и гладкой границей.

Эллиптичность краевой задачи с формально положительным оператором (1.10) можно также установить, обратившись к ее обобшенной постановке и следуюшему неравенству Корна. 
ПРЕДЛОЖЕНИЕ 1.11 ([12]). Если матрица $\Lambda\left(x, \nabla_{x}\right)$ алгебраически комплектна, то для функиии $и \in \mathscr{D}^{0} H(\Omega)$ справедливо неравенство

$$
\left\|u ; \mathscr{D}^{0} H(\Omega)\right\| \leqslant c\left(\left\|\Lambda u ; L_{2}(\Omega)\right\|+\left\|u ; L_{2}(\Omega)\right\|\right),
$$

в котором постоянная с зависит от $\Lambda$ и $\Omega$, но не от $u$.

Это же неравенство (1.33) позволяет рассмотреть несамосопряженные матричные операторы прежней структуры

$$
\mathscr{L}\left(x, \nabla_{x}\right)=\Lambda^{*}\left(x, \nabla_{x}\right)(A(x)+i B(x)) \Lambda\left(x, \nabla_{x}\right) .
$$

Здесь $i$ - мнимая единища, $A$ и $B$ - симметрические гладкие матришы-функции, $A(x)$ положительно определена при всех $x$. Разумеется, краевая задача $(1.8),(1.9)$ перестает быть формально самосопряженной, однако теорема 1.9 (2) сохраняется в полном объеме. Дело в том, что, во-первых, оператор краевых условий Неймана принимает вид

$$
\mathscr{N}\left(x, \nabla_{x}\right)=\mathscr{N}^{0}\left(x, \nabla_{x}\right)(A(x)+i B(x)) \Lambda\left(x, \nabla_{x}\right),
$$

во-вторых, переход к сопряженной задаче сводится к замене $+i$ на $-i$ в $(1.34),(1.35)$ и, в-третьих, согласно (1.23) линеал полиномов $\mathscr{P}$ аннулируется оператором $\Lambda\left(x, \nabla_{x}\right)$, т.е. оказывается одинаковым для обеих задач (подробности см. в [32]).

6. Конкретные задачи математической физики. Обсудим ряд задач, имеющих явную физическую природу. Количество примеров, конечно же, можно было бы увеличить, но мы ограничиваемся двумя разделами механики - теорией упругости и гидродинамикой, предоставляюшими иллюстративный материал и в следуюших параграфах.

ПримеР 1.12. Согласуя матричную запись уравнений линейной теории упругости (см. [53]-[55], [12] и др.) с обозначениями из п. 1, обнаруживаем, что $A(x)$ - матрица Гука, построенная по аналогичному тензору упругих модулей и потому являюшаяся симметрической и положительно определенной,

$$
\Lambda(\xi)^{t}=\left(\begin{array}{cccccc}
\xi_{1} & 0 & 0 & 0 & \alpha \xi_{3} & \alpha \xi_{2} \\
0 & \xi_{2} & 0 & \alpha \xi_{3} & 0 & \alpha \xi_{1} \\
0 & 0 & \xi_{3} & \alpha \xi_{2} & \alpha \xi_{1} & 0
\end{array}\right), \quad \alpha=\frac{1}{\sqrt{2}} .
$$

Таким образом, $T=T^{\prime}=3, t_{1}=t_{2}=t_{3}$ и $N=6$. Столбец $u=\left(u_{1}, u_{2}, u_{3}\right)^{t}$ интерпретируется как вектор смещений, а столбец

$$
A \Lambda u=\left(\sigma_{11}, \sigma_{22}, \sigma_{33}, \alpha^{-1} \sigma_{23}, \alpha^{-1} \sigma_{31}, \alpha^{-1} \sigma_{12}\right)^{t}
$$

содержит декартовы компоненты $\sigma_{j k}$ тензора напряжений.

Матрица (1.36) алгебраически комплектна и $\rho_{\Lambda}=2$ в $5^{0}$. Как нетрудно проверить, линеал полиномов (1.23) совпадает с пространством жестких смешений

$$
\mathscr{P}=\left\{p(x)=a+b \times x: a, b \in \mathbb{R}^{3}\right\}
$$


(крестом обозначено векторное произведение, все функции вешественные). Условия Дирихле $\mathscr{D} u \equiv u=0$ отвечают жестко зашемленной границе упругого тела, а условия Неймана - свободной или нагруженной, так как 3 -столбец $\mathcal{N}\left(x, \nabla_{x}\right) u(x)$ из $(1.13)$ является вектором $\sigma^{\nu}(u ; x)$ нормальных напряжений на поверхности $\partial \Omega$. В соответствии с правилом (1.6) можно соорудить смешанные краевые условия, для чего второе слагаемое из (1.2) удобно переписать в виде

$$
(\mathscr{N} u, \mathscr{D} v)_{\partial \Omega}=(\mathscr{U} \mathscr{N} u, \mathscr{U} \mathscr{D} v)_{\partial \Omega}
$$

и лишш потом оперировать со строками матриц $\mathscr{U} \mathscr{N}$ и $\mathscr{U} \mathscr{D}$; здесь $\mathscr{U}$ - произвольная унитарная матрица-функция на $\partial \Omega$. Впрочем, подобные условия редко поддаются физическому осмыслению.

Для изотропного однородного упругого тела матрища $A$ имеет вид

$$
A=\operatorname{diag}\left\{2 \mu \mathbb{I}_{3}+\lambda \mathbb{E}_{3}, 2 \mu \mathbb{I}_{3}\right\}
$$

где $\mu>0$ и $\lambda \geqslant 0$ - постоянные Ламе, $\mathbb{I}_{q}$ - единичная $(q \times q)$-матрица, а элементами $(q \times q)$-матрицы $\mathbb{E}_{q}$ служат $q^{2}$ единиц.

ПримеР 1.13. Деформированное состояние упругого электропроводящего тела (см., например, [56], [57]) описывается посредством системы дифференциальных уравнений с оператором $\mathscr{Q}\left(x, \nabla_{x}\right)=\Lambda\left(\nabla_{x}\right)^{*} Q(x) \Lambda\left(\nabla_{x}\right)$, где

$$
\begin{gathered}
\Lambda(\xi)^{t}=\left(\begin{array}{ccccccccc}
\xi_{1} & 0 & 0 & 0 & \alpha \xi_{3} & \alpha \xi_{2} & 0 & 0 & 0 \\
0 & \xi_{2} & 0 & \alpha \xi_{3} & 0 & \alpha \xi_{1} & 0 & 0 & 0 \\
0 & 0 & \xi_{3} & \alpha \xi_{2} & \alpha \xi_{1} & 0 & 0 & 0 & 0 \\
0 & 0 & 0 & 0 & 0 & 0 & \xi_{1} & \xi_{2} & \xi_{3}
\end{array}\right) \\
Q=\left(\begin{array}{cc}
Q_{11} & Q_{12} \\
Q_{21} & -Q_{22}
\end{array}\right), \quad \alpha=\frac{1}{\sqrt{2}}
\end{gathered}
$$

$Q_{11}$ и $Q_{22}$ - вещественные, симметрические, положительно определенные матрищы размера $6 \times 6$ и $3 \times 3$, а $Q_{12}=Q_{21}^{t}$ - вешественная $(6 \times 3)$-матрица. При этом $u=$ $\left(v_{1}, v_{2}, v_{3}, \varphi\right)^{t} ; \varphi$-электрический потенциал, а $v_{j}$-смещения точек тела вдоль осей $x_{j}$. Все группы краевых условий, допускаюшие физическое истолкование, образуются по правилу (1.6).

Алгебраическая комплектность матрицы (1.40) (расширенной матрицы (1.36)) сомнений не вызьвает. Вместе с тем, из-за "неправильного" знака при блоке $Q_{22}$ оператор $Q\left(x, \nabla_{x}\right)$ не является формально положительным. Однако, переобозначив $u=$ $\left(v_{1}, v_{2}, v_{3}, i \varphi\right)^{t}$ и

$$
A=\left(\begin{array}{cc}
Q_{11} & 0 \\
0 & Q_{22}
\end{array}\right), \quad B=\left(\begin{array}{cc}
0 & Q_{12} \\
Q_{21} & 0
\end{array}\right),
$$

приходим к эквивалентной системе дифференциальных уравнений с оператором (1.34), у которого все необходимые свойства имеются. Таким образом, для краевых задач электропезоупругости верна теорема 1.9, в которой

$$
\mathscr{P}=\mathscr{P}^{a}=\left\{p(x)=(a+b \times x, c): a, b \in \mathbb{R}^{3}, c \in \mathbb{R}\right\} .
$$


ПРИмеР 1.14. При рассмотрении пластины как двумерного объекта возникает система уравнений $(1.8)$, в которой $\Omega \subset \mathbb{R}^{2}, u=\left(u_{1}, u_{2}, u_{3}\right)^{t}$, а оператор $\mathscr{L}$ имеет вид (1.10), причем $A-(6 \times 6)$-матрица, симметрическая и положительно определенная, и

$$
\Lambda(\xi)=\left(\begin{array}{cccccc}
\xi_{1} & 0 & \alpha \xi_{2} & 0 & 0 & 0 \\
0 & \xi_{2} & \alpha \xi_{1} & 0 & 0 & 0 \\
0 & 0 & 0 & \alpha^{2} \xi_{1}^{2} & \alpha^{2} \xi_{2}^{2} & \xi_{1} \xi_{2}
\end{array}\right), \quad \alpha=\frac{1}{\sqrt{2}}
$$

т.е. $T=T^{\prime}=3, t_{1}=t_{2}=1, t_{3}=2$ и $N=6$. Матрица (1.41) алгебраически комплектна (отметим, что $\rho_{\Lambda}=2$ в $5^{0}$ ) и

$$
\mathscr{P}=\mathscr{P}^{a}=\left\{p(x)=\left(c_{1}-c_{0} x_{2}, c_{2}+c_{0} x_{1}, C_{0}+C_{1} x_{1}+C_{2} x_{2}\right): c_{j}, C_{j} \in \mathbb{R}\right\} .
$$

Как и в предыдущих примерах, каждой возникающей конструкции можно придать физический смысл, но мы ограничимся констатацией следующего: компоненты $u_{1}$ и $u_{2}$ столбца $u$ являются продольными смешениями в пластине, $u_{3}$ - поперечным, а краевые условия Дирихле

$$
u_{1}(x)=0, u_{2}(x)=0, u_{3}(x)=0, \partial_{\nu} u_{3}(x)=0, x \in \partial \Omega,
$$

означают жесткое зашемление края пластины. В (1.43) $\partial_{\nu}$ - производная вдоль внешней нормали $\nu=\left(\nu_{1}, \nu_{2}\right)$, а соответствуюший граничньй матричньй оператор вьглядит так:

$$
\mathscr{D}(x, \xi)=\left(\begin{array}{cccc}
1 & 0 & 0 & 0 \\
0 & 1 & 0 & 0 \\
0 & 0 & 1 & \nu_{1}(x) \xi_{1}+\nu_{2}(x) \xi_{2}
\end{array}\right)
$$

Мы еще вернемся к задачам теории пластин в $\S 2$ и $₫ 4$.

ПримеР 1.15. Оператор системы уравнений Стокса

$$
\begin{gathered}
-\Delta_{x} u(x)+\operatorname{grad} u^{\prime}(x)=f(x), \\
-\operatorname{div} u(x)=f^{\prime}(x), \quad x \in \Omega \subset \mathbb{R}^{3},
\end{gathered}
$$

допускает запись (1.14), в которой $T=3, T^{\prime}=4, t_{1}=t_{2}=t_{3}=1$ и $t_{4}=0$; кроме того, $m^{\prime}=m=3$ и

$$
\mathscr{L}\left(\nabla_{x}\right)=-\operatorname{diag}\left\{\Delta_{x}, \Delta_{x}, \Delta_{x}\right\}, \quad \mathscr{Q}^{*}\left(\nabla_{x}\right)=\nabla_{x} .
$$

При этом $\Delta_{x}=\nabla_{x} \cdot \nabla_{x}$ - оператор Лапласа, grad $=\nabla_{x}$ и $\operatorname{div}=\nabla_{x} \cdot-$ векторные операции; $u=\left(u_{1}, u_{2}, u_{3}\right)^{t}$-поле скоростей, а $u^{\prime}=u_{4}-$ гидростатическое давление в стационарном потоке жидкости.

Формулы Грина (1.2) и (1.17) конкретизируются так:

$$
\begin{gathered}
\left(-\Delta_{x} u, v\right)_{\Omega}+\left(\partial_{\nu} u, v\right)_{\Omega}=\sum_{j=1}^{3}\left(\nabla_{x} u_{j}, \nabla_{x} v_{j}\right)_{\Omega} \equiv a(u, v ; \Omega) \\
\left(\nabla_{x} u^{\prime}, v\right)_{\Omega}=\left(u^{\prime},-\nabla_{x} \cdot v\right)_{\Omega}+\left(\nu u^{\prime}, v\right)_{\partial \Omega}
\end{gathered}
$$


Следовательно, в (1.16) $\mathscr{D}=\mathscr{D}^{\prime}=\mathbb{I}_{3}, \mathscr{N}=\mathscr{N}^{\prime}=\operatorname{diag}\left\{\partial_{\nu}, \partial_{\nu}, \partial_{\nu}\right\}, \mathscr{K}=\nu^{t}$; матришы $\mathscr{M}$ и $\mathscr{S}$ становятся пустыми и $\left(\mathscr{N}^{\prime}, \mathscr{K}\right) \tilde{u}=\partial_{\nu} u+\nu u^{\prime}$, где $\nu=\left(\nu_{1}, \nu_{2}, \nu_{3}\right)^{t}-$ единичный вектор внешней нормали. Таким образом, формула Грина (1.19) теряет нестандартные интегралы с $\mathscr{S}$ и приобретает классический вид (см., например, [58]):

$$
\begin{aligned}
& \left\{\left(-\Delta_{x} u+\nabla_{x} u^{\prime}, v\right)_{\Omega}+\left(-\nabla_{x} \cdot u, v^{\prime}\right)_{\Omega}\right\}+\left(\partial_{\nu} u+\nu u^{\prime}, v\right)_{\partial \Omega} \\
& =\left\{\left(u,-\Delta_{x} v+\nabla_{x} v^{\prime}\right)_{\Omega}+\left(u^{\prime},-\nabla_{x} \cdot v\right)\right\}+\left(u, \partial_{\nu} v+\nu v^{\prime}\right)_{\partial \Omega} .
\end{aligned}
$$

Как показано в [58], граничные интегралы $\left(\partial_{\nu} u+\nu u^{\prime}, v\right) \partial \Omega$ и т.п. можно заменить в (1.50) суммами $\left(u, \tau^{(\nu)}(\mathbf{v})\right)_{\partial \Omega}-\left(\nu \nabla_{x} \cdot u, v^{\prime}\right)_{\partial \Omega}$ и т.п., вернув формуле Грина строение (1.19). Здесь $\tau^{(\nu)}=\left(\tau_{1}^{(\nu)}, \tau_{2}^{(\nu)}, \tau_{3}^{(\nu)}\right)^{t}$ - вектор нормальных напряжений,

$$
\tau_{j}^{(\nu)}=\sum_{h=1}^{3} \nu_{h} \tau_{j h}, \quad \tau_{j k}(\mathbf{u})=e_{j k}(u)-\delta_{j k} u^{\prime}, \quad e_{j k}(u)=\frac{\partial u_{j}}{\partial x_{k}}+\frac{\partial u_{k}}{\partial x_{j}} .
$$

Из (1.48) вытекает, что $\mathscr{P}^{a}=\mathbb{R}^{3}$. Так как алгебраическая комплектность матрицы $\mathscr{Q}^{*}(\xi)=\xi$ сомнений вызвать не может, предложение 1.7 устанавливает полиномиальное свойство $1^{0}$ пары $\{\mathscr{L}, a\}$ из (1.47) и (1.48), причем $\mathscr{P}=\mathbb{R}^{4}$. Прежний прием (1.38), привлекающий унитарную матрицу $\mathscr{U}$, позволяет помимо условий прилипания $u=0$ на $\partial \Omega$ включить в изложенную схему линейную часть условий на свободной поверхности

$$
v \cdot u=0, \quad \nu \times \tau^{(\nu)}(\mathbf{u})=0 \text { на } \partial \Omega
$$

(проекция вектора $\nu \times \tau^{(\nu)}$ на направление $\nu$ равна нулю тождественно и поэтому в (1.52) по сушеству присутствуют три краевых условия).

ПримеР 1.16. Рассмотрим двумерную линеаризованную систему, описьвающую течение мультиполярной жидкости

$$
-\frac{\partial}{\partial x_{1}} t_{1 k}(\mathbf{u})-\frac{\partial}{\partial x_{2}} t_{2 k}(\mathbf{u})=f_{k}, \quad k=1,2 ; \quad-\nabla_{x} \cdot u=f_{3} \quad \text { в } \quad \Omega .
$$

Здесь $\mathbf{u}=\left(u_{1}, u_{2}, u_{3}\right)^{t}, u_{1}$ и $u_{2}-$ скорости, $u^{\prime}=u_{3}$ - давление, $t_{j k}$ - модифицированные напряжения (моментные), $h>0$ - параметр,

$$
t_{j k}(\mathbf{u})=e_{j k}(u)-\delta_{j k} u^{\prime}-h \Delta_{x} e_{j k}(u), \quad j, k=1,2
$$

(см. (1.51)). Для простоты некоторым физическим константам приданы конкретные значения (ср. с [59]). При $h=0$ и $f_{3}=0$ соотношения (1.52) не отличаются от двумерных уравнений Стокса (1.45), (1.46). Оператор системы (1.51) записывается в виде (1.14), где $T=2, T^{\prime}=3, t_{1}=t_{2}=2, t_{3}=-1, m=4, m^{\prime}=3$ и

$$
\mathscr{L}(\xi)=2\left[h|\xi|^{2}+1\right]\left(\begin{array}{cc}
|\xi|^{2}+\xi_{1}^{2} & \xi_{1} \xi_{2} \\
\xi_{2} \xi_{1} & |\xi|^{2}+\xi_{2}^{2}
\end{array}\right), \quad \mathcal{Q}^{*}(\xi)=\left(\begin{array}{c}
\xi_{1} \\
\xi_{2}
\end{array}\right) .
$$


Многократньм интегрированием по частям (в том числе и вдоль контура $\partial \Omega$ ) выводим формулу Грина (1.2), в которой

$$
\begin{gathered}
a(u, v ; \Omega)=\frac{1}{2}\left(e_{j k}(u), e_{j k}(v)\right)_{\Omega}+\frac{h}{2}\left(\nabla_{x} e_{j k}(u), \nabla_{x} e_{j k}(v)\right)_{\Omega}, \\
\mathscr{D} v=\left(v_{1}, v_{2}, \partial_{\nu} v_{1}, \partial_{\nu} v_{2}\right), \quad \mathscr{N} u=\left(\mathscr{N}_{1} u, \mathscr{N}_{2} u, \mathscr{N}_{3} u, \mathscr{N}_{4} u\right), \\
\mathscr{N}_{2+p} u=h \nu_{j} \nu_{k} \frac{\partial}{\partial x_{k}} e_{j p}(u), \\
\mathscr{N}_{p} u=\nu_{j}\left[e_{j p}(u)-h \Delta_{x} e_{j p}(u)\right]-h \partial_{s}\left[s_{j} \nu_{k} \frac{\partial}{\partial x_{k}} e_{j p}(u)\right] .
\end{gathered}
$$

Индексы $j, k$ и $p$ принимают значения 1 и $2 ;$ по $j$ и $k$ производится суммирование. Кроме того, $\nu=\left(\nu_{1}, \nu_{2}\right)^{t}$ и $s=\left(s_{1}, s_{2}\right)^{t}$ - единичные векторы нормали и касательной; $\partial_{\nu}=\nu \cdot \nabla_{x}$ и $\partial_{s}=s \cdot \nabla_{x}$.

Для формы $a$ из (1.54) выполняется требование $2^{0}$ и $\mathscr{P}^{a}=\left\{p(x)=\left(c_{1}-c_{0} x_{2}\right.\right.$, $\left.\left.c_{2}+c_{0} x_{1}\right): c_{q} \in \mathbb{R}\right\}$. Поскольку $a^{0}(u, v ; \Omega)=2^{-1} h\left(\nabla_{x} e_{j k}(u), \nabla_{x} e_{j k}(v)\right)_{\Omega}$, полиномиальное свойство выдерживает замораживание коэффициентов, однако линеал полиномов расширяется и уже содержит все линейные функции. Предложение 1.7 наделяет полиномиальным свойством $1^{0}$ пару $\left\{\mathscr{L}^{\prime}, a\right\}$.

Для применения теоремы 1.9 об эллиптичности задач в $\Omega$ конкретизируем процедуру построения операторов краевых условий, изложенную в п. 2. Необходимая формула Грина (1.17) указана в (1.49). С целью вьвести представление (1.16) воспользуемся приемом (1.38) и заменим в (1.54) операторы $\mathscr{D}$ и $\mathscr{N}$ такими:

$$
\begin{aligned}
\mathscr{D} v & =\left(v_{1}, v_{2}-\nu_{2} \partial_{\nu} v_{1}+\nu_{1} \partial_{\nu} v_{2}, \nu_{1} \partial_{\nu} v_{1}+\nu_{2} \partial_{\nu} v_{2}\right), \\
\mathscr{N} u & =\left(\mathscr{N}_{1} u, \mathscr{N}_{2} u,-\nu_{2} \mathscr{N}_{3} u+\nu_{1} \mathscr{N}_{4} u, \nu_{1} \mathscr{N}_{3} u+\nu_{2} \mathscr{N}_{4} u\right) .
\end{aligned}
$$

Теперь $(3 \times 4)$-матрицу $\mathscr{D}^{\prime}$ в $(1.16)$ составим из строк $\mathscr{D}_{1}, \mathscr{D}_{2}, \mathscr{D}_{3}$, отвечающих первым трем выражениям в правой части (1.55); аналогично из $\mathscr{N}$ конструируется $\mathscr{N}^{\prime}$. Очевидная формула

$$
\nu_{1} \partial_{\nu} v_{1}+\nu_{2} \partial_{\nu} v_{2}=\nabla_{x} \cdot v+\nu_{2} \partial_{s} v_{1}-\nu_{1} \partial_{s} v_{2}
$$

дает требуемое представление для $\mathscr{D}_{4}$; при этом в $(1.16) \mathscr{S}=1$ (скаляр) и $\mathscr{M}=$ $\left(\nu_{2} \partial_{s},-\nu_{1} \partial_{s}, 0\right)$ (строка). Наконец, последнее слагаемое из (1.49) переписывается в виде $\left(\mathscr{K} u^{\prime}, \mathscr{D}^{\prime} v\right)_{\partial \Omega}$, требуемом в $(1.17)$ и содержашем столбец $\mathscr{K}=\left(\nu_{1}, \nu_{2}, 0\right)^{t}$. Итак, в соответствии с (1.18) конструируются те краевые условия, которые включаются в предложенную схему и в большинстве своем допускают физическую интерпретацию. Отметим, что в силу (1.16) и (1.18) условия Дирихле для системы (1.53) принимают вид

$$
v_{1}=0, \quad v_{2}=0, \quad-\nu_{2} \partial_{\nu} v_{1}+\nu_{1} \partial_{\nu} v_{2}=0 \text { на } \partial \Omega
$$

и содержат одним соотношением менњше, чем условия $\mathscr{D} v=0$ для оператора $\mathscr{L}$ (см. $(1.54))$. Если к (1.56) добавить уравнение неразрывности $-\nabla_{x} \cdot v=0$, суженное на $\partial \Omega$, то возникнет система Дирихле, равносильная $\mathscr{D}$. 


\section{§. Краевые задачи в областях с кусочно гладкой границей}

1. Область с коническими точками. Предположим, что граница $\partial \Omega$ области $\Omega \subset \mathbb{R}^{n}$ гладкая всюду, кроме точек $x^{1}, \ldots, x^{M}$, составляющих множество нерегулярностей $\Upsilon$. Пусть при любом $\mu=1, \ldots, M$ сушествует диффеоморфизм окрестности $\mathscr{V}^{\mu} \ni x^{\mu}$ на шар $\mathbb{B}_{1}^{n}\left(x^{\mu}\right)=\left\{x:\left|x-x^{\mu}\right|<1\right\}$, переводящий $\Omega \cap \mathscr{V}^{\mu}$ в $\mathbb{K}^{\mu} \cap \mathbb{B}_{1}^{n}\left(x^{\mu}\right)$, где $\mathbb{K}^{\mu}$ - конус с вершиной $x^{\mu}$, вырезаюший на единичной сфере $\mathbb{S}^{n-1}\left(x^{\mu}\right)=\partial \mathbb{B}_{1}^{n}\left(x^{\mu}\right)$ область $\omega^{\mu}$ с гладкой $(n-2)$-мерной границей $\partial \omega^{\mu}$ (не исключается случай $\partial \omega^{\mu}=\varnothing$, когда $x^{\mu}$ - изолированная точка границы и $\mathbb{K}^{\mu}=\mathbb{R}^{n} \backslash x^{\mu}$-полный конус). Упрошая обозначения, но не ограничивая обшности, считаем, что $\mathscr{V}^{\mu} \cap \Omega=\mathbb{B}_{1}^{n}\left(x^{\mu}\right) \cap \mathbb{K}^{\mu}$.

ЗАмЕчАниЕ 2.1. В этом параграфе рассматриваются ограниченные области, но в принципе пересказ результатов для областей с коническими выходами на бесконечность не составляет труда - далее в $\S 5$ обсуждается один из возможных вариантов.

Пусть оператор $\mathscr{L}$ с простой или усложненной структурой и соответствующая форма $a$ обладают полиномиальным свойством $1^{0}$, выдерживающим замораживание коэффициентов в любой точке $x^{0} \in \bar{\Omega}$. Образуем граничный оператор $\mathscr{B}$ в согласии с правилом (1.6) или (1.18) и рассмотрим аналогичную $(1.8),(1.9)$ краевую задачу

$$
\begin{aligned}
& \mathscr{L}\left(x, \nabla_{x}\right) u(x)=f(x), \quad x \in \Omega, \\
& \mathscr{B}\left(x, \nabla_{x}\right) u(x)=q(x), \quad x \in \partial \Omega \backslash \Upsilon .
\end{aligned}
$$

Следуя [13], введем пространство $V_{\beta}^{l}(\Omega, \Upsilon)$ как пополнение $C_{0}^{\infty}(\bar{\Omega} \backslash \Upsilon)$ по норме

$$
\left\|w ; V_{\beta}^{l}(\Omega, \Upsilon)\right\|=\left(\sum_{k=0}^{l}\left\|\rho^{\beta-l+k} \nabla_{x}^{k} w ; L_{2}(\Omega)\right\|^{2}\right)^{1 / 2}
$$

где $\rho(x)=\operatorname{dist}\{x, \Upsilon\}$, a $l \in\{0,1, \ldots\}$ и $\beta \in \mathbb{R}-$ показатели гладкости и веса. Если $l>0$, то $V_{\beta}^{l-1 / 2}(\partial \Omega, \Upsilon)$ - следовое пространство, снабженное естественной нормой

$$
\left\|W ; V_{\beta}^{l-1 / 2}(\partial \Omega, \Upsilon)\right\|=\inf \left\{\left\|w ; V_{\beta}^{l}(\Omega, \Upsilon)\right\| \mid W=w \text { на } \partial \Omega \backslash \Upsilon\right\}
$$

Определим еще векторные пространства, похожие на (1.26) и (1.27),

$$
\begin{aligned}
\mathscr{D}_{\beta}^{l} V(\Omega) & =\prod_{k=1}^{T} V_{\beta}^{l+t_{k}}(\Omega, \Upsilon) \\
\mathscr{R}_{\beta}^{l} V(\Omega) & =\prod_{j=1}^{T} V_{\beta}^{l-t_{j}}(\Omega, \Upsilon) \times \prod_{q=1}^{m} V_{\beta}^{l-r_{q}-1 / 2}(\partial \Omega, \Upsilon) .
\end{aligned}
$$

В левых частях этих формул указывается только область $\Omega$, поскольку для нее множества $\partial \Omega$ и $\Upsilon$ уже зафиксированы. Сокрашая обозначения, далее пишем $V_{\beta}^{l}(\Omega)$ и т. п.

Если для $l$ выполнено неравенство (1.28), оператор задачи (2.1), (2.2),

$$
\mathscr{A}_{\beta}^{l}=\{\mathscr{L}, \mathscr{B}\}: \mathscr{D}_{\beta}^{l} V(\Omega) \rightarrow \mathscr{R}_{\beta}^{l} V(\Omega)
$$


оказывается непрерьвным при любом $\beta$. С целью сформулировать условия его фредгольмовости, перепишем главные части $\mathscr{L}^{\mu}\left(\nabla_{x}\right)$ и $\mathscr{B}^{\mu}\left(x, \nabla_{x}\right)$, вычисленные в точке $x^{\mu}$, при помоши сферических координат $\left(\rho_{\mu}, \varphi^{\mu}\right)$, где $\rho_{\mu}=\left|x-x^{\mu}\right|$, а $\varphi^{\mu}-$ координаты точки в карте подходящего атласа на сфере $\mathbb{S}^{n-1}\left(x^{\mu}\right)$. Для элементов матриц имеем

$$
\begin{aligned}
& \mathscr{L}_{j k}^{\mu}\left(\nabla_{x}\right)=\rho_{\mu}^{-t_{j}-t_{k}} L_{j k}^{\mu}\left(\varphi^{\mu}, \nabla_{\varphi^{\mu}}, \partial / \partial \rho_{\mu}\right), \\
& \mathscr{B}_{q k}^{\mu}\left(\nabla_{x}\right)=\rho_{\mu}^{-t_{j}-r_{q}} B_{q k}^{\mu}\left(\varphi^{\mu}, \nabla_{\varphi^{\mu}}, \partial / \partial \rho_{\mu}\right)
\end{aligned}
$$

Подчеркнем, что из-за искривленности конической поверхности $\partial \mathbb{K}^{\mu}$ коэффициенты граничного оператора $\mathscr{B}^{\mu}$ остаются переменными, но он допускает запись (2.8). Заменив дифференцирование $\partial / \partial \rho_{\mu}$ величиной $\lambda-t_{k}$, положим

$$
\begin{aligned}
& L^{\mu}\left(\varphi, \partial_{\varphi}, \lambda\right)=\left(L_{j k}^{\mu}\left(\varphi, \partial_{\varphi}, \lambda-t_{k}\right)\right)_{j, k=1}^{T}, \\
& B^{\mu}\left(\varphi, \partial_{\varphi}, \lambda\right)=\left(B_{q k}^{\mu}\left(\varphi, \partial_{\varphi}, \lambda-t_{k}\right)\right)_{q, k=1}^{m, T}
\end{aligned}
$$

и введем полиномиальный пучок (спектральное семейство)

$$
\mathbb{C} \ni \lambda \mapsto \mathfrak{A}^{\mu}(\lambda)=\left\{L^{\mu}\left(\varphi, \partial_{\varphi}, \lambda\right), B^{\mu}\left(\varphi, \partial_{\varphi}, \lambda\right)\right\}: \mathscr{D}^{l} H\left(\omega^{\mu}\right) \rightarrow \mathscr{R}^{l} H\left(\omega^{\mu}\right) .
$$

Известно (см. [4]), что его спектр $\sigma\left(\mathfrak{A}^{\mu}\right)$ состоит из изолированных конечнократных собственных чисел, лежащих в объединении двойного угла и полосы

$$
\{\lambda \in \mathbb{C}:|\operatorname{Im} \lambda|<\gamma|\operatorname{Re} \lambda| \text { или }|\operatorname{Re} \lambda|<h\} \quad(\gamma, h>0) .
$$

ЗАмечание 2.2. 1) Обычно на место $\lambda$ в (2.9) ставят $-i \lambda$, что вызвано использованием преобразования Меллина (замена Эйлера $\rho \mapsto t=\log \rho$ и преобразование Фурье $\left.\mathscr{F}_{t \rightarrow \lambda}\right)$. Поскольку технические детали у нас не затрагиваются, удобно избавиться от "лишнего" множителя $-i$. В связи с этим множество (2.11) меняет ориентацию и содержит вешественную ось вместо мнимой.

2) Имеется возможность сопоставить каждой точке $x \in \Upsilon$ свой весовой показатель $\beta(x)$, изменив тем самым норму (2.3). Для наших целей достаточно оперировать с постоянным $\beta$, и упомянутым обобшением здесь пренебрегаем.

Согласно [13] оператор (2.7) оказывается фредгольмовым в том и только том случае, когда прямая $i \mathbb{R}-(\beta-l+n / 2) \equiv\{\lambda \in \mathbb{C}: \operatorname{Re} \lambda=l-\beta-n / 2\}$ не содержит собственных чисел пучков $\mathfrak{A}^{1}, \ldots, \mathfrak{A}^{M}$. Если же хотя бы одно собственное число попадает на указанную прямую, образ $\operatorname{Im} \mathscr{A}_{\beta}^{l}=\mathscr{A}_{\beta}^{l} \mathscr{D}_{\beta}^{l} V(\Omega)$ становится незамкнутым в $\mathscr{R}_{\beta}^{l} V(\Omega)$, но $\operatorname{dim}$ ker $\mathscr{A}_{\beta}^{l}$, dim coker $\mathscr{A}_{\beta}^{l}<\infty$.

Полиномиальное свойство $3^{0}$ формы $a^{\mu}$ с замороженными в $x^{\mu}$ коэффициентами, отвечающей модельной задаче в конусе $\mathbb{K}^{\mu}$ :

$$
\mathscr{L}^{\mu}\left(\nabla_{x}\right) \mathscr{U}(x)=\mathscr{F}(x), x \in \mathbb{K}^{\mu} ; \mathscr{B}^{\mu}\left(x, \nabla_{x}\right) \mathscr{U}(x)=\mathscr{G}(x), x \in \partial \mathbb{K}^{\mu} \backslash x^{\mu}
$$

позволяет получить полную информацию о спектре $\sigma\left(\mathfrak{A}^{\mu}\right)$ пучка $\mathfrak{A}^{\mu}$ в узкой полосе

$$
\Pi^{\mu}=\left\{\lambda \in \mathbb{C}:|\operatorname{Re} \lambda+n / 2|<\delta_{\mu}\right\}
$$


и сделать заключение о разрешимости задач $(2.1),(2.12)$ в классах $\mathscr{D}_{\beta}^{l} V(\Omega), \mathscr{D}_{\beta}^{l} V\left(\mathbb{K}^{\mu}\right)$ при

$$
\beta-l \in(-\delta, \delta), \quad \delta=\min \left\{\delta_{1}, \ldots, \delta_{M}\right\}>0
$$

Введем необходимые обозначения. Пусть $\mathscr{P}^{\mu}$ - линеал, отвечающий паре $\left\{\mathscr{L}^{\mu}, a^{\mu}\right\}$ согласно $1^{0}-3^{0}$. Обобщенная степень $\operatorname{deg}\{p ; \mathbf{t}\}$ (векторного) полинома $p=\left(p_{1}, \ldots, p_{T}\right)$ равна $q$, если $\operatorname{deg} p_{j}=q+t_{j}$ для (скалярных) полиномов $p_{j}$. Этот полином однороден относительно точки $x^{\mu}$, если $p_{j}$ - однородные полиномы переменной $x-x^{\mu}$. Подчеркнем, что обобщенная степень может быть отрицательной и

$$
\operatorname{deg}\{p, \mathbf{t}\} \geqslant-\max \mathbf{t}=-\max \left\{t_{1}, \ldots, t_{T}\right\}
$$

ПРЕДЛОЖЕНИЕ 2.3 ([27]). Существует такое $\delta_{\mu} \in(0,1)$, что верны следующие утверждения.

1) Если пара $\left\{\mathscr{L}^{\mu}, a^{\mu}\right\}$ обладает полиномиальным свойством $1^{0}$, то в полосе $\Pi^{\mu}$ может располагаться разве лишь собственное число $\lambda_{0}=-n / 2$ пучка (2.10). Собственное подпространство ker $\mathfrak{A}^{\mu}\left(\lambda_{0}\right)$ состоит из следов на $\omega^{\mu} \subset \mathbb{S}^{n-1}\left(x^{\mu}\right)$ полиномов из линеала

$$
\begin{array}{r}
\mathscr{P}_{0}^{\mu}=\left\{p \in \mathscr{P}^{\mu}: \mathscr{B}^{\mu} p=0 \text { на } \partial \mathbb{K}^{\mu} \backslash x^{\mu}, \operatorname{deg}\{p ; \mathbf{t}\}=-n / 2,\right. \\
\left.p \text { однороден относительно } x^{\mu}\right\} .
\end{array}
$$

2) Если $\varkappa_{\mu}=\operatorname{dim} \mathscr{P}_{0}^{\mu}>0$ и форма а ${ }^{\mu}$ обладает полиномиальньм свойством в полном обвеме, то полная алгебрачческая кратность собственного числа $\lambda_{0}=$ -n/2 пучка (2.10) равна $2 \varkappa_{\mu}$ и любая жсорданова чепочка имеет длину 2.

Нетрудно указать ситуации, в которых линеал (2.15) заведомо тривиален и пересечение $\sigma\left(\mathfrak{A}^{\mu}\right) \cap \Pi^{\mu}$ пусто.

СлЕДСТВИЕ 2.4. В условиях предложсения 2.3 (1) полоса (2.13) свободна от спектра пучка $\mathfrak{A}^{\mu}$, если выполнено одно из трех условий:

1) $n$ - нечетное число,

2) $\max \mathbf{t}<n / 2$,

3) $\mathscr{B}^{\mu}=\mathscr{D}^{\mu}-$ система Дирихле на $\partial \mathbb{K}^{\mu} \backslash x^{\mu}$.

Предложение $2.3(2)$ означает, что каждый собственньй вектор $\Phi^{0} \in \operatorname{ker} \mathfrak{A}^{\mu}\left(\lambda_{0}\right)=$ $\left.\mathscr{P}_{0}^{\mu}\right|_{\omega^{\mu}}$ сопровождается присоединенньм $\Phi^{1}$ и цепочка $\Phi^{0}, \Phi^{1}$ непродолжима, т.е. нет присоединенных векторов второго порядка. Разумеется, $\Phi^{0}, \Phi^{1} \in C^{\infty}\left(\overline{\omega^{\mu}}\right)$. Кроме того, функции $\mathbb{K}^{\mu} \ni x \mapsto$

$$
\mathscr{U}^{0}(x)=\rho_{\mu}^{\mathbf{t}-n / 2} \Phi^{0}\left(\varphi^{\mu}\right), \quad \mathcal{U}^{1}(x)=\rho_{\mu}^{\mathbf{t}-n / 2}\left(\Phi^{0}\left(\varphi^{\mu}\right) \log \rho_{\mu}+\Phi^{1}\left(\varphi^{\mu}\right)\right)
$$

удовлетворяют однородной задаче (2.12) и являются ее степенными решениями вида

$$
\mathscr{U}(x)=\rho_{\mu}^{\lambda+\mathbf{t}} \Phi\left(\varphi^{\mu}\right)=\left(\rho_{\mu}^{\lambda+t_{1}} \Phi_{1}\left(\varphi^{\mu}, \log \rho_{\mu}\right), \ldots, \rho_{\mu}^{\lambda+t_{T}} \Phi_{T}\left(\varphi^{\mu}, \log \rho_{\mu}\right)\right),
$$


где $l \mapsto \Phi_{j}(\cdot, l)$ - полиномы с коэффициентами из $C^{\infty}\left(\overline{\omega^{\mu}}\right)$. Функция $x \mapsto \mathscr{U}^{0}(x)$ оказывается полиномом из $\mathscr{P}_{0}^{\mu}$, аннулируюшим форму $a^{0}$, а для $\mathscr{U}^{1}$ интеграл $a^{0}\left(\mathscr{U}^{1}, \mathscr{U}^{1} ; \mathbb{K}^{\mu}\right)$ расходится как на бесконечности, так и в точке $x^{\mu}$.

Поясним идею доказательства предложения 2.3 (1) на примере оператора простой структуры. При $\lambda \in i \mathbb{R}-n / 2$ степенное решение $\mathscr{U}$ из (2.17) подставляется на обе позиции в формулу Грина (1.2) на сферическом слое $\Xi=\left\{x \in \mathbb{K}^{\mu}: b_{-}<\rho_{\mu}<b_{+}\right\}$, где $b_{+}>b_{-}>0$. Очевидно, что $\left(\mathscr{L}^{\mu} \mathscr{U}, \mathscr{U}\right)_{\Xi}=0$. Интеграл по боковой поверхности $\Gamma=\partial \Xi \cap \partial \mathbb{K}^{\mu}$ также исчезает, поскольку один из сомножителей в каждом члене суммы

$$
\left(\mathscr{N}^{\mu} \mathscr{U}, \mathscr{D}^{\mu} \mathscr{U}\right)_{\Gamma}=\sum_{\mu=1}^{q}\left(\mathcal{N}_{q}^{\mu} \mathscr{U}, \mathscr{D}_{q}^{\mu} \mathscr{U}\right)_{\Gamma}
$$

равен нулю согласно определению (1.6) и равенству $\mathscr{B}^{\mu} \mathscr{U}=0$ на $\Gamma \subset \partial \mathbb{K}^{\mu}$. В левой части формулы (1.2) остались два интеграла $I_{ \pm}$по сферическим областям $\omega_{ \pm}^{\mu}=$ $\left\{x \in \mathbb{K}^{\mu}: \rho_{\mu}=b_{ \pm}\right\}$. Благодаря условию $\operatorname{Re} \lambda=-n / 2$ (мнимые части $\lambda$ сокращаются из-за комплексного сопряжения второго сомножителя) и в согласии с замечанием 1.2 подьнтегральные выражения $J_{ \pm}$оказьваются положительно однородными функциями степени $1-n$, а значит, $J_{ \pm}(x)= \pm a_{ \pm}^{1-n} J_{0}(x)$ (мы учли разнонаправленность нормалей к $\left.\omega_{ \pm}^{\mu}\right)$. Следовательно, $I_{+}+I_{-}=0$ и $a^{\mu}(\mathscr{U}, \mathscr{U} ; \Xi)=0$, т.е. $\mathscr{U} \in \mathscr{P}^{\mu}$ в силу $1^{0}$. Отсюда вытекает, что $\mathscr{U}$ попадает в линеал (2.15), а $\lambda=\lambda_{0}$ - единственно возможная точка спектра $\mathfrak{A}^{\mu}$ на прямой $i \mathbb{R}-n / 2$. Переход к полосе (2.13) обеспечивается обшими свойствами спектра пучка (см. текст между (2.10) и (2.11)).

В точности так же обрабатьваются операторы с усложненной структурой. При подозрении на неправомочность использования формулы Грина в области $\Xi$ с кусочно гладкой границей можно привлечь прием умножения на "сглаженную ступеньку" $\chi_{\delta}\left(\rho_{\mu}\right)$, обсуждавшийся в п. $5 \S 1$.

Если $\sigma\left(\mathfrak{A}_{\mu}\right) \cap \Pi_{\mu}=\varnothing$, то согласно [13] при ограничениях (1.28) и (2.14) на показатели $l_{\text {и }} \beta$ оператор: $\mathscr{D}_{\beta}^{l} V\left(\mathbb{K}^{\mu}\right) \rightarrow \mathscr{R}_{\beta}^{l} V\left(\mathbb{K}^{\mu}, x^{\mu}\right)$ задачи $(2.12)$ в конусе $\mathbb{K}^{\mu}$ оказывается изоморфизмом. Это свойство модельных задач (при всех $\mu=1, \ldots, M$ ), а также эллиптичность самой задачи (2.1), (2.2), проверенная в теореме 1.9, влекут фредгольмовость отображения (2.7). В случае $-n / 2 \in \sigma\left(\mathfrak{A}_{\mu}\right)$ на интервале $(l-\delta, l+\delta)$ запретным становится показатель $\beta=l$. Более детальная информация об отображении (2.7), дающая описание подпространств ker $\mathscr{A}_{\beta}^{l}$ и coker $\mathscr{A}_{\beta}^{l}$ в терминах линеала полиномов $\mathscr{P}(\mathscr{B})$, получается при помоши очередных двух утверждений обшего характера.

ПРЕДЛОЖЕНИЕ 2.5 ([15], [21] и [5], [8], [52]). Операторы $\mathscr{A}_{\beta}^{l}$ u $\mathscr{A}_{2 l-\beta}^{l}$ фредгольмовы одновременно, причем при наличии этого свойства

$$
\operatorname{coker} \mathscr{A}_{\beta}^{l}=\left\{\left(v, \mathscr{T} v, \mathscr{N}^{\prime \prime} v\right): v \in \operatorname{ker} \mathscr{A}_{2 l-\beta}^{l}\right\} \text {. }
$$

Иными словами, задачи $(2.1),(2.2)$, поставленные в пространствах $\mathscr{D}_{\beta}^{l} V(\Omega)$ и $\mathscr{D}_{2 l-\beta}^{l} V(\Omega)$, сопряжены относительно формулы Грина (1.19) (или (1.7) - cp. с (1.30) и с комментарием по поводу матриц простой структуры). Поэтому коядро одной задачи определяется ядром другой: замена $\beta \mapsto 2 l-\beta$ переставляет операторы в (2.18). При изучении свойств отображения (2.7) в зависимости от весового показателя $\beta$ решающую роль играет “формула о приращении индекса”, упрощенньй вариант которой приводится ниже. 
ПРЕДЛОЖЕНИЕ 2.6 ([60], [15], [20], [21]). Пусть $\beta^{+} \in(l, l+\delta) u \beta^{-} \in(l-\delta, l)$. Тогда справедливо равенство

$$
\text { Ind } \mathscr{A}_{\beta^{+}}^{l}=\operatorname{Ind} \mathscr{A}_{\beta^{-}}^{l}+2\left(\varkappa_{1}+\cdots+\varkappa_{M}\right) \text {, }
$$

где Ind $A=\operatorname{dim} \operatorname{ker} A-\operatorname{dim} \operatorname{coker} A-$ индекс оператора $A$, а последнее слагаемое - суммарная алгебраическая кратность собственного числа $\lambda_{0}=-n / 2$ пучков $\mathfrak{A}^{1}, \ldots \mathfrak{A}^{M}\left(\right.$ ecлu $\lambda_{0} \notin \sigma\left(\mathfrak{A}^{\mu}\right)$, mo $\left.\varkappa_{\mu}=\operatorname{dim} \mathscr{P}_{0}^{\mu}=0\right)$.

Сформулируем центральные утверждения данного раздела.

ТЕорема 2.7 ([30], [31; 6.1$])$. Предположим, что оператор $\mathscr{L}$ и форма а в задаче (2.1), (2.2) обладают полиномиальным свойством, выдержсивающим замораживание в любой точке $x^{0} \in \bar{\Omega}$.

1) Если при каждом $\mu=1, \ldots, M$ линеал полиномов (2.15) тривиален $\left(\varkappa_{1}=\cdots=\varkappa_{M}=0 ;\right.$ см., например, следствие 3.4$)$, то для показателей $l$ и $\beta$, подчиненных (1.28) и (2.14),

$$
\begin{aligned}
&(2.20) \quad \operatorname{ker} \mathscr{A}_{\beta}^{l}=\mathscr{P}_{+}(\mathscr{B}) \equiv\{p=\left(p_{1}, \ldots, p_{T}\right) \in \mathscr{P}(\mathscr{B}): \nabla_{x}^{s} p_{j}\left(x^{\mu}\right)=0 \\
&\left.n p u s \leqslant t_{j}-n / 2, \mu=1, \ldots, M, j=1, \ldots, T\right\}, \\
&(2.21) \quad \operatorname{coker} \mathscr{A}_{\beta}^{l}=\left\{\left(p, \mathscr{T}_{p}, \mathscr{N}^{\prime \prime} p\right): p \in \mathscr{P}_{+}(\mathscr{B})\right\} ;
\end{aligned}
$$

здесь подпространство $\mathscr{P}(\mathscr{B}) \subset \mathscr{P}$ определено в согласии с (1.29).

2) Предположим дополнительно, что в случае $\varkappa_{\mu}>0$ у формы а $a^{\mu}$ имеется полиномиальное свойство $3^{0}$. Тогда при $\beta^{-} \in(l-\delta, l)$ ядро оператора $\mathscr{A}_{\beta}^{l}$ определено равенством (2.20), в котором $\beta=\beta^{-}$. Если же $\beta \in(l, l+\delta)$, то

$$
\operatorname{ker} \mathscr{A}_{\beta^{+}}^{l}=\operatorname{ker} \mathscr{A}_{\beta^{-}}^{l} \dot{+} \mathscr{Z}
$$

где $\mathscr{Z}$ - подпространство, размерность которого равна $\varkappa_{1}+\cdots+\varkappa_{M}$, а для элементов Ц верны формуль

$$
\zeta-\mathscr{U}^{1 \mu}-\mathscr{U}^{0 \mu} \in \mathscr{D}_{\beta^{-}}^{l} V\left(\Omega \cap \mathscr{V}^{\mu}, \partial \Omega \cap \mathscr{V}^{\mu}, x^{\mu}\right), \quad \mu=1, \ldots, M
$$

$\left(\mathscr{U}^{0 \mu}\right.$ и $\mathscr{U}^{1 \mu}$ - степенные решения (2.16) модельных задач (2.12)). Кроме того, $\mathscr{Z}$ содержит подпространство полиномов

$$
\begin{aligned}
\mathscr{P}_{0}(\mathscr{B})= & \{p \in \mathscr{P}(\mathscr{B}): \operatorname{deg}\{p ; \mathbf{t}\}=-n / 2, \\
& \text { p однороден относительно каждой из точек } \left.x^{1}, \ldots, x^{M}\right\},
\end{aligned}
$$

а в асимптотике (2.22) решения $\zeta \in \mathscr{Z} \backslash \mathscr{P}_{0}(\mathscr{B})$ присутствует хотя бы один нетривиальный логарифмический член $\mathscr{U}^{1 \mu}$. Наконеи, представления для coker $\mathscr{A}_{\beta^{ \pm}}$ получаются в соответствии с (2.18).

Формулы Грина (1.2) и (1.20), справедливые изначально для $u, v \in C_{0}^{\infty}(\bar{\Omega} \backslash \Upsilon)^{T}$, по замьканию переносятся на пары $u \in \mathscr{D}_{\beta}^{l} V(\Omega), v \in \mathscr{D}_{2 l-\beta}^{l} V(\Omega)$. Поэтому при $\beta \leqslant l$ доказательство равенства (2.20) достигается так же, как и в п. $5 \S 1$ : функция $u \in \operatorname{ker} \mathscr{A}_{\beta}^{l} \subset \mathscr{D}_{\beta}^{l} V(\Omega) \subset \mathscr{D}_{2 l-\beta}^{l} V(\Omega)$ подставляется на обе позиции в $(1.2)$ или $(1.20)$ и 
применяется требование $1^{0}$. Проверка остальных утверждений чуть более сложна и требует обрашения к соотношениям $(2.18),(2.19)$ и теореме об асимптотике решений [13], [31; $§ 4.2]$. Подчеркнем, что при $\beta \leqslant l$ класс $\mathscr{D}_{\beta}^{l} V(\Omega)$ содержится в энергетическом пространстве $\mathscr{D}^{0} H(\Omega)$, порожденном формой $a$ и неравенством Корна (см. предложение 1.11 и соотношение (1.12)).

В теореме 2.7 не было задействовано множество

$$
\mathscr{P}_{-}(\mathscr{B})=\{p \in \mathscr{P}(\mathscr{B}): \operatorname{deg}\{p ; \mathbf{t}\}<-n / 2\}
$$

которое состоит из полиномиальных решений однородной задачи $(2.1),(2.2)$ и не обязано быть тривиальным (так, для условий Неймана $\mathscr{P}(\mathscr{N})=\mathscr{P}$ в силу $4^{0}$ и $\operatorname{dim} \mathscr{P}_{-}(\mathscr{N})>0$ в случае $\left.\max \mathbf{t}>n / 2\right)$. Если $\mathscr{P}_{-}(\mathscr{B}) \neq\{0\}$, то $\delta_{\mu}$ в предложении 2.3 оказывается таким, что условие (2.14) исключает обсуждаемые полиномы из $\mathscr{D}_{\beta}^{l} V(\Omega)$. Причина кажушейся неувязки в том, что при $\operatorname{dim} \mathscr{P}_{-}(\mathscr{B})>0$ весовые классы из теоремы 2.7 соответствуют задаче Соболева $(2.1),(2.2)$ с дополнительными условиями

$$
\nabla_{x}^{s} u_{j}\left(x^{\tau}\right)=0, s<t_{j}-n / 2, j=1, \ldots, T ; \quad \mu=1, \ldots, M
$$

(см. [61], [31; 112.7$]$ и др.).

Переход к обычным “энергетическим” решениям $u \in \mathscr{D}^{0} H(\Omega)$ может быть осуществлен в рамках теории самосопряженных расширений (см. далее п. 7).

Как показьвает анализ доказательств, предложение 2.3 (2) и, следовательно, теорема 2.7 (2) сохраняют силу и для операторов усложненной структуры (1.14), если в формуле Грина (1.20) удается избавиться от поверхностного интеграла за счет одного из предположений $\mathbf{u} \in \mathscr{P}(\mathscr{B})$ или $\mathbf{v} \in \mathscr{P}(\mathscr{B})$.

Пример 2.8. Снабдим систему Стокса (1.45), (1.46) условиями прилипания $\mathscr{D}^{\prime} u \equiv$ $u=0$ на $\partial \Omega$. Тогда $\mathscr{P}\left(\mathscr{D}^{\prime}, 0\right)=\{p=(0,0,0, c): c \in \mathbb{R}\}$ (см. пример 1.15) и согласно (1.48), (1.49) при соленоидальном поле скоростей $v \in C^{\infty}(\bar{\Omega})^{3}$ получаем

$$
\left(-\nabla_{x} u+\nabla_{x} u^{\prime}, v\right)_{\Omega}+\left(\partial_{\nu} u+\nu u^{\prime}, v\right)_{\partial \Omega}=a(u, v ; \Omega) .
$$

Если $v \in \mathscr{P}\left(\mathscr{D}^{\prime}, 0\right)$, то интеграл по $\partial \Omega$ в (2.26), конечно же, равен нулю, т.е. задача Стокса приобретает некое подобие свойства $4^{0}$, причем роль оператора $\mathscr{N}$ в $4^{0}$ передается системе Дирихле $\mathscr{D}^{\prime}$. Аналогичное заключение можно сделать и для задачи (1.53), (1.56) о мультиполярной жидкости. Впрочем, это не находит применения в данном контексте, поскольку и для плоских и для трехмерных течений упомянутые задачи обслуживаются утверждениями 2.3 (1) и 2.7 (1). Полезность обсуждаемого обобшения станет понятной в п. $5 \S 3$.

Поскольку и другие примеры были бы прямым пересказом общей теоремы, упомянем лишь, что в п. 7 мы вернемся к рассмотрению задачи теории пластин.

2. Область с гладким ребром (некритический случай). Обратим внимание на качественное расхождение результатов, установленных в теореме 2.7. В ситуации $\operatorname{dim} \mathscr{P}_{0}^{\mu}=0(\mu=1, \ldots, M)$ при условии (2.14) Ind $\mathscr{A}_{\beta}^{l}=0$ (cp. (2.20) и (2.21)). Если же хотя бы один линеал $\mathscr{P}_{0}^{\mu}$ из (2.15) нетривиален (критическая ситуация), то 
Ind $\mathscr{A}_{\beta}^{l} \neq 0$ при любом допустимом $\beta$. В самом деле, $\beta \mapsto$ Ind $\mathscr{A}_{\beta}^{l}$ - монотонная функция и в силу теоремы $2.7(2) \pm$ Ind $\mathscr{A}_{\beta}^{l}=2\left(\varkappa_{1}+\cdots+\varkappa_{M}\right)>0$ при $\pm(\beta-l) \in(0, \delta)$.

Указанное обстоятельство становится решающим при изучении особых подмногообразий $\Upsilon \subset \partial \Omega$ с положительной размерностью: в критической ситуации оператор $\mathscr{A}_{\beta}^{l}$ задачи (2.1), (2.2) перестает быть фредгольмовым (при любом $\left.\beta \in \mathbb{R} !\right)$, так как одно из подпространств ker $\mathscr{A}_{\beta}^{l}$ или coker $\mathscr{A}_{\beta}^{l}$ оказывается бесконечномерньм. В данном разделе будет рассмотрен некритический случай $\operatorname{dim} \mathscr{P}_{0}^{\mu}=0$, а в следующих двух - продемонстрированы способы изменения весовых классов, приводящие к фредгольмовым операторам в критическом случае. Подчеркнем, что формулируемые далее теоремы 2.13 и 2.17 относятся также к ситуации $d=\operatorname{dim} \Upsilon=0$ (конические точки) и доставляют оператор задачи (2.1), (2.2) с нулевьм индексом.

Пусть $n>2$ и $\Upsilon \subset \partial \Omega$ - гладкое замкнутое подмногообразие без края, $\operatorname{dim} \Upsilon=d \in$ $(0, n-2]$. Предположим, что поверхность $\partial \Omega$ гладкая всюду, кроме $\Upsilon$, и для каждой точки $x^{0} \in \Upsilon$ имеется диффеоморфизм ее окрестности $\mathscr{V}\left(x^{0}\right)$ на шар $\mathbb{B}_{1}^{n}$, переводяший $\Omega \cap \mathscr{V}\left(x^{0}\right)$ на $\mathbb{D}\left(x^{0}\right) \cap \mathbb{B}_{1}^{n}$, где $\mathbb{D}\left(x^{0}\right)=\mathbb{K}\left(x^{0}\right) \times \mathbb{R}^{d}$ и $\mathbb{K}\left(x^{0}\right)$ есть $(n-d)$-мерный конус, вырезаюший на сфере $\mathbb{S}^{n-d-1}$ область $\omega\left(z^{0}\right)$ с гладкой $(n-d-2)$-мерной границей. В случае $n=3, d=1$ мы имеем дело с двугранным углом $\mathbb{D}$, а при $\partial \omega=\varnothing$ конус $\mathbb{K}$ полньй и подмногообразие $\Upsilon$ является внутренней частью компакта $\bar{\Omega}$. Далее вместо $\mathbb{D}\left(x^{0}\right)$ и т.п. пишем $\mathbb{D}^{0}$ и т.п., но помним, что $\mathbb{D}^{0}, \mathbb{K}^{0}$ и $\omega^{0}$ переменные. Декартову систему координат $(y, z)$ фиксируем так, чтобы $y \in \mathbb{K}^{0}$ и $z \in \mathbb{R}^{d}$.

Задаче (2.1), (2.2) сопоставим оператор (2.7), определенньй на пространствах (2.5), (2.6). Заморозив коэффициенты дифференциальных операторов и формы $a$ в точке $x^{0} \in \Upsilon$, перейдем к модельной задаче

$$
\begin{aligned}
\mathscr{L}^{0}\left(\nabla_{y}, \nabla_{z}\right) u(y, z) & =f(y, z), & & (y, z) \in \mathbb{D}^{0}, \\
\mathscr{B}^{0}\left(y, \nabla_{y}, \nabla_{z}\right) u(y, z) & =g(y, z), & & (y, z) \in \partial \mathbb{D}^{0} \backslash \Upsilon^{0},
\end{aligned}
$$

где $\Upsilon^{0}=\{0\} \times \mathbb{R}^{d}$ - спрямленное ребро. Применим преобразование Фурье $\mathscr{F}_{z \rightarrow \xi}$ и сделаем замену $y \mapsto \eta=|\xi|^{-1} y$. В результате получаем эталонную краевую задачу в конусе $\mathbb{K}^{0}$, зависящую от параметра $\theta=|\xi|^{-1} \xi \in \mathbb{S}^{d-1}$,

$$
\begin{aligned}
\mathscr{L}^{0}\left(\nabla_{\eta}, i \theta\right) U(\eta, \theta) & =F(\eta, \theta), & & \eta \in \mathbb{K}^{0}, \\
\mathscr{B}^{0}\left(\eta, \nabla_{\eta}, i \theta\right) U(\eta, \theta) & =G(\eta, \theta), & & \eta \in \partial \mathbb{K}^{0} \backslash\{0\},
\end{aligned}
$$

где $|\xi|^{-t_{j}} U_{j}(|\xi| y, \theta),|\xi|^{t_{j}} F_{j}(|\xi| y, \theta),|\xi|^{\sigma_{q}} G_{q}(|\xi| y, \theta)$ - образы Фурье компонент $u_{j}(y, z)$, $f_{j}(y, z), g_{q}(y, z)$ функций $u, f, g$. Форма $a_{\theta}^{0}\left(u, v ; \mathbb{K}^{0}\right)$, наследуемая задачей $(2.28)$ от исходной задачи $(2.1),(2.2)$, имеет вид

$$
\sum_{j, k=1}^{T} \sum_{|\sigma|=t_{j}} \sum_{|\gamma|=t_{k}} \int_{\Xi} \stackrel{\circ}{a}_{j k}^{\sigma \gamma}\left(\nabla_{\eta}, i \theta\right)^{\gamma} u_{k}(\eta)\left(\nabla_{\eta},-i \theta\right)^{\sigma} \overline{v_{j}(\eta)} d \eta
$$

где $\stackrel{\circ}{a} \underset{j k}{\sigma \gamma}$ - постоянные (уже замороженные в точке $x^{0}$ ) коэффициенты формы $a^{0}$ и

$$
\left(\nabla_{\eta}, i \theta\right)=\left(\partial / \partial \eta_{1}, \ldots, \partial / \partial \eta_{n-d}, i \theta_{1}, \ldots, i \theta_{d}\right) .
$$

Если в (2.29) положить $\theta_{1}=\cdots=\theta_{d}=0$, то возникнет форма $a_{0}^{0}$, отвечаюшая модельной задаче вида $(2.12)$ в конусе $\mathbb{K}^{0} \subset \mathbb{R}^{n-d}$ с операторами $\mathscr{L}^{0}\left(\nabla_{\eta}, 0\right)$ и $\mathscr{B}^{0}\left(\eta, \nabla_{\eta}, 0\right)$, удовлетворяющими соотношениям $(2.8)$, в которых $\mu=0, \rho_{0}=|\eta|$ и $\varphi^{0}$ - координаты на $\mathbb{S}^{n-d-1}$ (обрашаем внимание на понижение размерности). 
Лемма 2.9 ([27]). Пусть у пары $\left\{\mathscr{L}^{0}\left(\nabla_{y}, \nabla_{z}\right), a^{0}\right\}$ имеется полиномиальное свойство $1^{0}$ с линеалом РР0.

1) Пара $\left\{\mathscr{L}^{0}\left(\nabla_{\eta}, 0\right), a_{0}^{0}\right\}$ наследует свойство $1^{0}$, причем соответствующий линеал полиномов $P^{0}$ определяется так:

$$
P^{0}=\left\{p(\eta): p \in \mathscr{P}, p(y, z) \text { не зависит от } z \in \mathbb{R}^{d}\right\} .
$$

2) $E с л и \Xi \subset \overline{\mathbb{K}}^{0}, U \in C^{\infty}(\bar{\Xi})^{T}$ и $a_{\theta}^{0}(U, U ; \Xi)=0, \mathscr{L}^{0}\left(\nabla_{\eta}, i \theta\right) U=0$ на $\Xi$ при $\theta \in \mathbb{S}^{d-1}$, mo $U=0$.

Подобные заключения делаются и для полиномиальных свойств $2^{0}$ и $3^{0}$.

Проверка утверждений достигается подстановкой функции $\Xi \times \mathbb{B}_{1}^{d} \ni(y, z) \mapsto$ $U(y) \exp \left(i\left[\theta_{1} z_{1}+\cdots+\theta_{d} z_{d}\right]\right)$ в формулы из $1^{0}$.

Следуя [22], введем пространство $E_{\beta}^{l}\left(\mathbb{K}^{0}\right)$ как пополнение $C_{0}^{\infty}\left(\overline{\mathbb{K}^{0}} \backslash\{0\}\right)$ по норме

$$
\left\|w ; E_{\beta}^{l}\left(\mathbb{K}^{0}\right)\right\|=\left(\sum_{k=0}^{l}\left\|\rho_{0}^{\beta-l+k}\left(1+\rho_{0}\right)^{l-k} \nabla_{\eta}^{k} w ; L_{2}\left(\mathbb{K}^{0}\right)\right\|^{2}\right)^{1 / 2} .
$$

Подчеркнем, что вблизи вершины конуса распределение весовых множителей в (2.31) такое же, как и в норме $\left\|w ; V_{\beta}^{l}\left(\mathbb{K}^{0}, 0\right)\right\|$ из $(2.3)$, однако около бесконечности множители становятся величинами $O\left(\rho_{0}^{\beta}\right)$, не зависяшими от порядка $k$ производной. Изменение весовой нормы вызвано присутствием младших членов в дифференшиальных операторах из $(2.28)$ - задача $\left\{\mathscr{L}^{0}\left(\nabla_{\eta}, q \theta\right), \mathscr{B}^{0}\left(\eta, \nabla_{\eta}, q \theta\right)\right\}$ оказьвается эллиптической с параметром $q \in \mathbb{R}$ (см. [4]). Формулы (2.4)-(2.6) помогают определить следовые $E_{\beta}^{l-1 / 2}\left(\partial \mathbb{K}^{0}\right)$ и векторные $\mathscr{D}_{\beta}^{l} E\left(\mathbb{K}^{0}\right), \mathscr{R}_{\beta}^{l} E\left(\mathbb{K}^{0}\right)$ пространства, в которых оператор задачи $(2.8)$,

$$
A_{\beta}^{l}(\theta)=\left\{\mathscr{L}^{0}\left(\nabla_{\eta}, i \theta\right), \mathscr{B}^{0}\left(\eta, \nabla_{\eta}, i \theta\right)\right\}: \mathscr{D}_{\beta}^{l} E\left(\mathbb{K}^{0}\right) \rightarrow \mathscr{R}_{\beta}^{l} E\left(\mathbb{K}^{0}\right),
$$

является непрерывньм при всех $\beta \in \mathbb{R}$ и $l$, подчиненных (1.28).

ПРЕДЛОЖЕНИЕ 2.10 ([27]). Пусть в дополнение $к$ условию леммы 2.9 линеал

$$
\begin{array}{r}
P_{0}^{0}=\left\{p \in P^{0}: \mathscr{B}^{0}\left(\eta, \nabla_{\eta}, 0\right) p(\eta)=0, \eta \in \partial \mathbb{K}^{0} \backslash 0 ; \operatorname{deg}\{p ; \mathbf{t}\}=-(n-d) / 2,\right. \\
\text { р однороден относительно точки } \eta=0\}
\end{array}
$$

(ср. с (2.15)) тривиален. Тогда найдется такое $\delta_{0}>0$, что при $|\beta-l|<\delta_{0}$ отображения (2.32) и

$$
\left\{\mathscr{L}^{0}\left(\nabla_{y}, \nabla_{z}\right), \mathscr{B}^{0}\left(y, \nabla_{y}, \nabla_{z}\right)\right\}: \mathscr{D}_{\beta}^{l} V\left(\mathbb{D}^{0}, \Upsilon^{0}\right) \rightarrow \mathscr{R}_{\beta}^{l} V\left(\mathbb{D}^{0}, \partial \mathbb{D}^{0}, \Upsilon^{0}\right)
$$

суть изоморфизмы.

При учете леммы 2.9 (2) и общих свойств оператора (2.32) (см. [22], [23], [20; § 6.2]) соотношения $\operatorname{dim} \operatorname{ker} A_{\beta}^{l}(\theta)=0$ и $\operatorname{dim} \operatorname{coker} A_{\beta}^{l}(\theta)=0$ выводятся так же, как и представления (2.20) и (2.21). Переход к оператору (2.34) модельной задачи в $\mathbb{D}^{0}$ обеспечивается обшими результатами [22], [23], которые к тому же гарантируют фредгольмовость отображения $(2.7)$ в случае

$$
|\beta-l|<\delta=\min \left\{\delta_{0} \equiv \delta\left(x^{0}\right): x^{0} \in \Upsilon \subset \partial \Omega\right\} .
$$

Дальнейшие рассуждения не отличаются от намеченных в п. 1. 
Tеорема 2.11 ([27]). Пусть $\beta$ и l удовлетворяют (2.35) u (1.28), пара $\{\mathscr{L}, a\}$ обладает полиномиальным свойством, выдерживающим замораживание коэффичиентов всюду в $\bar{\Omega}$, и, кроме того, для любой точки $x^{0} \in \Upsilon$ равна нулю размерность линеала $P_{0}\left(x^{0}\right)$, построенного согласно (2.33). Тогда оператор (2.7) задачи (2.1), (2.2) фредгольмов, а его ядро и коядро находятся по формулам (2.20) и (2.21), в которых роль $\mathscr{P}_{+}(\mathscr{B})$ играет линеал

$$
\begin{aligned}
\mathscr{P}_{*}(\mathscr{B})=\left\{p \in \mathscr{P}(\mathscr{B}): \nabla_{x}^{s} p_{j}\left(x^{0}\right)=\right. & 0, x^{0} \in \Upsilon \\
& \left.s<t_{j}-(n-d) / 2, j=1, \ldots, T\right\} .
\end{aligned}
$$

ЗАмЕчАниЕ 2.12. Если в (2.36) $\Upsilon$ - множество конических точек $\left\{x^{1}, \ldots, x^{M}\right\}$, то согласно $(2.23)$ и $(2.20) \mathscr{P}_{*}(\mathbb{B})=\mathscr{P}_{+}(\mathscr{B}) \dot{+} \mathscr{P}_{0}(\mathscr{B})$. Разумеется, $\operatorname{dim} \mathscr{P}_{0}(\mathscr{B})=0$ ввиду условия $\operatorname{dim} P_{0}\left(x^{0}\right)=0$, т.е. указанная конкретизация теоремы 2.11 не отличается от теоремы $2.7(2)$. Обозначение $\mathscr{P}_{*}(\mathscr{B})$ удобно для следующих разделов.

Следствие 2.4, где $n$ заменено на $n-d$, содержит достаточные условия для выполнения равенств $\operatorname{dim} P_{0}\left(x^{0}\right)=0, x^{0} \in \Upsilon \subset \partial \Omega$. Упомянутые в примере 2.8 задачи о трехмерных течениях управляются теоремой 2.11. То же касается и задачи Дирихле (зашемленная граница) для систем уравнений теории упругости (примеры 1.12 и 1.13), однако для смешанных краевых задач условия теоремы 2.11 могут нарушаться (см. далее пример 2.14).

3. Область с гладким ребром (задача Неймана). Пусть $\mathscr{L}$ имеет простую структуру и $\mathscr{B}=\mathscr{N}$ - оператор Неймана из формулы Грина (1.2). Если $n-d-$ нечетное число или $n-d>2 \max \mathbf{t}$, то в силу следствия $2.4(1,2)$ и теоремы 2.11 при ограничении (2.35) оператор (2.7) задачи Неймана фредгольмов с нулевым индексом.

Пусть теперь $n-d$ четное и $n-d \leqslant 2 \max \mathbf{t}$. Считаем, что форма $a^{0}$ обладает полиномиальным свойством в полном объеме. По предложению 1.5 линеал $P_{0}\left(x^{0}\right)$ содержит все полиномы подходящей степени и потому нетривиален (размерность $\operatorname{dim} P_{0}\left(x^{0}\right)$ вычислить нетрудно). Таким образом, в шкале пространств $\mathscr{D}_{\beta}^{l} V(\Omega)$ не удается реализовать задачу Неймана как фредгольмов оператор. Поясним идею [25]-[27] исправления ситуации, базирующуюся на следующих наблюдениях (для простоты $\Upsilon$ - одна коническая точка $x^{1}=0$ ). Во-первых, отображение

$$
\{\mathscr{L}, \mathscr{N}\}: \mathbf{D}=\mathscr{D}_{\beta}^{l} V(\Omega)+\mathscr{P}_{0}^{1} \rightarrow \mathscr{R}_{\beta}^{l} V(\Omega)
$$

сохраняет непрерывность при $\beta \in(l-1, l)$ (для главных частей $\left\{\mathscr{L}^{1}, \mathscr{N}^{1}\right\} \mathscr{P}_{0}^{1}=0$ согласно $\left.4^{0}\right)$. Во-вторых, благодаря предложению $2.3(2)$ и формуле $(2.19)$ индекс оператора (2.37) становится нулевьм. В-третьих, по-прежнему $\mathbf{D} \subset \mathscr{D}^{0} H(\Omega)$ и для функций $u, v \in \mathbf{D}$ верна формула Грина (1.2), позволяюшая эксплуатировать полиномиальное свойство. Наконец, дифференцирование $\nabla_{x}^{k}$ при $k>t_{j}-(n-d) / 2$ уничтожает компоненты $p_{j}$ полиномов $p \in \mathscr{P}_{0}^{1}$, но оставляет нетривиальньпи компоненты $\mathscr{U}_{j}^{1}$ степенных решений (2.16) с логарифмами. Последнее наблюдение принципиально: функции $\mathscr{U}^{1}$ "отделяются" от полиномов $\mathscr{U}^{0}$ при помоши ступенчатых весовых норм.

Введем необходимые обозначения. Пусть $l$ и $s$ - целые числа, $l \geqslant 0$ и $s \leqslant l$. Пространство $V_{\beta}^{l, s}(\Omega)$ получается как пополнение $C_{0}^{\infty}(\bar{\Omega} \backslash \Upsilon)$ по норме

$$
\left\|w ; V_{\beta}^{l, s}(\Omega)\right\|=\left(\sum_{k=0}^{l}\left\|\rho^{\beta-l+k+\Theta(s-k)} \nabla_{x}^{k} w ; L_{2}(\Omega)\right\|^{2}\right)^{1 / 2},
$$


где $\Theta$ - функция Хевисайда, $\Theta(t)=1$ для $t \geqslant 0$ и $\Theta(t)=0$ для $t<0$. Если $s<0$, то нормы (2.3) и (2.38) совпадают. Однако при $s \geqslant 0$ функция $k \mapsto \beta-l+k+\Theta(s-k)$ является кусочно линейной (имеет "ступеньку" на уровне $k=s$ ), в то время как для нормы (2.3) график функции $k \mapsto \beta-l+k$ - наклонная прямая. Аналогично (2.5) и (1.26) положим

$$
\mathscr{D}_{\beta}^{l,(d-n) / 2} V(\Omega)=\prod_{k=1}^{T} V_{\beta}^{l+t_{k}, t_{k}-(n-d) / 2}(\Omega)
$$

и рассмотрим такой оператор задачи Неймана (2.1), (2.2):

$$
\mathscr{A}_{\beta}^{l,(d-n) / 2}=\{\mathscr{L}, \mathscr{N}\}: \mathscr{D}_{\beta}^{l,(d-n) / 2} V(\Omega) \rightarrow \mathscr{R}_{\beta}^{l} V(\Omega) .
$$

Teорема 2.13 ([26]). Пусть $n-d-$ четное число, не большее 2 max t. Предположим, что у $\{\mathscr{L}, a\}$ имеется полиномиальное свойство $1^{0}$, выдерживающее замораживание коэффициентов всюду в $\bar{\Omega}$, и для любой точки $x^{0} \in \Upsilon$ форма $a^{0}$, отвечающая модельной задаче в $\mathbb{D}\left(x^{0}\right)$, удовлетворяет $3^{0}\left(u 4^{0}\right)$. Тогда найдется такое $\delta \in(0,1)$, что при $\beta \in(l-\delta, l)$ u $l \leqslant \max \mathbf{t}$ оператор (2.39) задачи Неймана $\{\mathscr{L}, \mathscr{N}\}$ в $\Omega$ является фредгольмовым, причем его ядро и коядро находятся по формулам (2.2) и (2.21), где под $\mathscr{P}_{+}(\mathscr{B})$ подразумевается линеал $\mathscr{P}_{*}(\mathscr{N})$ из $(2.36)$.

Итак, построен фредгольмов оператор, обладаюший теми же характеристиками, что и оператор, доставляемьй теоремой 2.11 в некритическом случае.

ПримеР 2.14. Трехмерные задачи теории упругости с краевыми условиями Неймана (см. примеры 1.12 и 1.13 ) обслуживаются теоремой 2.13. Рассмотрим один из вариантов смешанной краевой задачи. Пусть $\Omega \subset \mathbb{R}_{+}^{3}-$ тело, лежашее на плоскости $\Pi=\left\{x: x_{3}=0\right\}$ и ограниченное сверху гладкой поверхностью $\Sigma$, пересекающей $\Pi$ вдоль гладкого контура $\Upsilon \ni x^{0}$ под углами $\alpha\left(x^{0}\right) \neq 0, \pi$. Уравнения равновесия

$$
\Lambda\left(-\nabla_{x}\right)^{t} A(x) \Lambda\left(\nabla_{x}\right) u(x)=f(x), \quad x \in \Omega,
$$

записанные при помощи матрицы (1.36), дополним условиями

$$
\begin{gathered}
\sigma^{\nu}(u ; x) \equiv \Lambda(\nu(x))^{t} A(x) \Lambda\left(\nabla_{x}\right) u(x)=g(x), \quad x \in \Sigma_{+}=\Sigma \cap \mathbb{R}_{+}^{3}, \\
\sigma_{13}(u ; x)=0, \quad \sigma_{23}(u ; x)=0, \quad u_{3}(x)=0, \quad x \in \Pi_{\Sigma},
\end{gathered}
$$

где $\Pi_{\Sigma}$ - основание тела, ограниченное контуром $\Upsilon$, а $\sigma_{j k}$ - декартовы компоненты тензора напряжений. Соотношения (2.42) суть линеаризованные условия Синњорини (контакт без трения); на тело $\Omega$ действуют объемные силы $f$ и поверхностные нагрузки $g$. Условие $u_{3}=0$ на $\Pi_{\Sigma}$ сужает линеал $\mathscr{P}_{0}=\mathbb{R}^{3}$ до $\mathbb{P}_{0}\left(x^{0}\right)=\mathbb{R}^{2} \times\{0\}$ и потому ступенчатые нормы нужны лишь для компонент $u_{1}$ и $u_{2}$ вектора смещений $u$. В остальном проверка фредгольмовости оператора задачи (2.40)-(2.42),

$$
V_{\beta}^{l+1,0}(\Omega)^{2} \times V_{\beta}^{l+1}(\Omega) \rightarrow V_{\beta}^{l-1}(\Omega)^{3} \times V_{\beta}^{l-1 / 2}\left(\Sigma_{+}\right)^{3} \times V_{\beta}^{l-1 / 2}\left(\Pi_{\Sigma}\right)^{2} \times V_{\beta}^{l+1 / 2}\left(\Pi_{\Sigma}\right),
$$

следует доказательству теоремы 2.13. При этом $l \in\{1,2, \ldots\}$ и $\beta \in(l-\delta, l)$, где число $\delta=\min \left\{\delta\left(x^{0}\right): x^{0} \in \Upsilon\right\} \in(0,1)$ зависит от формы поверхности $\Sigma$ (вычисление $\delta\left(x^{0}\right)$ 
по раствору $\alpha\left(x^{0}\right)$ двугранного угла можно найти, например, в [62], [63]). Линеал $\mathscr{P}_{*}=\left\{\left(c_{1}-c_{0} x_{2}, c_{2}+c_{0} x_{1}, 0\right): c_{q} \in \mathbb{R}\right\}$ содержит жесткие смещения тела $\Omega$ вдоль плоскости П, а условия разрешимости задачи (2.40)-(2.42),

$$
(f, p)_{\Omega}+(g, p)_{\Sigma_{+}}=0 \quad \forall p \in \mathscr{P}_{*}
$$

означают самоуравновешенность нагрузок в направлениях, параллельных П.

ЗАмЕчАниЕ 2.15. Предыдуший пример подсказьвает, что в качестве ребра $\Upsilon$ может выступать линия смены типа краевого условия (в том числе и на гладкой поверхности $\partial \Omega \subset \mathbb{R}^{3}$ ). Можно убедиться в том, что для справедливости теоремы 2.11 достаточна постановка условий Дирихле по одну сторону от $\Upsilon$. Поэтому совмешение условий прилипания на $\Sigma_{+}$с условиями $(1.52)$ на свободной поверхности $\Pi_{\Sigma}$ не требует обрашения к ступенчатым нормам.

4. Область с гладким ребром (пространства с отделенной асимптотикой). Прием из предыдушего раздела малоудобен, если пространство $P_{0}\left(x^{0}\right)$ зависит от $x^{0} \in \Upsilon$ (так будет, если в примере $2.14 \Upsilon$ - пространственная дуга). Опишем подход, применимьй в более общей ситуации. С этой целью воспроизведем лемму 8.4.8 из [30].

Лемма 2.16. Пусть $u \in \mathscr{D}_{\beta}^{l,(d-n) / 2} V\left(\mathbb{D}^{0}, \Upsilon^{0}\right), \beta \in(l-1, l) u\left\{p^{(1)}, \ldots, p^{(\mathbb{J})}\right\}$ - некоторый базис в линеале полиномов обобщенной степени $-(n-d) / 2$ (т.е. $\left.\operatorname{deg}\left\{p^{j} ; \mathbf{t}\right\}=(d-n) / 2\right)$. Тогда справедливо представление

$$
u(y, z)=\sum_{j=1}^{\mathbb{J}} p^{(j)}(y)\left(\mathfrak{C}^{0} k_{j}\right)(|y|, z)+\tilde{u}(y, z)
$$

әде коэффициенты $k_{j}$ и остаток й удовлетворяют неравенству

$$
\left\|\tilde{u} ; \mathscr{D}_{\beta}^{l} V\left(\mathbb{D}^{0}, \Upsilon^{0}\right)\right\|+\sum_{j=1}^{\mathbb{J}}\left\|k_{j} ; H^{l-\beta}\left(\mathbb{R}^{d}\right)\right\| \leqslant c\left\|u ; \mathscr{D}_{\beta}^{l,(d-n) / 2} V\left(\mathbb{D}^{0}, \Upsilon^{0}\right)\right\|,
$$

а $\mathfrak{C}^{0}$ - оператор продолжения с ребра $\Upsilon^{0}$ в область $\mathbb{D}^{0}$, определенный, например, равенством

$$
\left(\mathfrak{C}^{0} k\right)(\rho, z)=\int_{\mathbb{R}^{d}} X(\zeta) k(z-\rho \zeta) d \zeta
$$

в котором $X(z)=(2 \pi)^{-d / 2} \mathscr{F}_{\xi \rightarrow z}^{-1} \chi(|\xi|)$ (обратное преобразование Фурье) $и \chi \in$ $C_{0}^{\infty}\left(\overline{\mathbb{R}}_{+}\right)$- эталонная срезка, $\chi(\rho)=1$ при $\rho \leqslant 1$.

Левая часть (2.43) представляет собой эквивалентную норму в $\mathscr{D}_{\beta}^{l,(d-n) / 2} V\left(\mathbb{D}^{0}, \Upsilon^{0}\right)$, и поэтому разумно в качестве области определения оператора задачи $(2.1),(2.2)$ взять весовое пространство функиий с отделенной асимптотикой. Введем необходимые обозначения и сформулируем теорему, вытекающую из более общих результатов $[64],[65]$. 
В трубчатой окрестности $V$ подмногообразия $\Upsilon$ введем координаты $(y, z)$, где $y \in$ $\mathbb{K}\left(x^{0}\right)$, а через $z$, несколько вольно, обозначаются координаты точки $x^{0}$ в подходящей карте атласа на $\Upsilon$. При помоши достаточно мелкого разбиения единишы на $\mathscr{V}$ “склеим” оператор $\mathfrak{C}$ продолжения функций с $\Upsilon$ в $\Omega$ из эталонных операторов $(2.44)$. Прямое описание подобных операторов и проверку их основных свойств можно найти, например, в [66]-[68].

Предположим, что размерность $J=\operatorname{dim} \mathbb{P}_{0}\left(x^{0}\right)>0$ линеала (2.33) постоянна на $\Upsilon$ и, более того, можно ввести базис $\left\{p^{1}, \ldots, p^{J}\right\}$ полиномов $y \mapsto p^{j}(y, z)$ в $\mathbb{P}_{0}\left(x^{0}\right)$, гладко зависящих от точки $x^{0} \in \Upsilon$ (и от координаты $z$ ). При $\beta-l \in(-1,0)$ определим пространство $\mathbf{D}_{\beta}^{l} V(\Omega)$ как множество функций, представимых в виде

$$
u(x)=\sum_{j=1}^{J} p^{(j)}(y, z)\left(\mathfrak{C} k_{j}\right)(|y|, z)+\tilde{u}(x),
$$

где $k_{j} \in H^{l-\beta}(\Upsilon)$ и $\tilde{u} \in \mathscr{D}_{\beta}^{l} V(\Omega)$. Подчеркнем, что согласно определению $\mathfrak{C}$ сумма из (2.45) исчезает вне $\mathscr{V}$. Нормой в $\mathbf{D}_{\beta}^{l} V(\Omega)$ назначим выражение

$$
\left\|u ; \mathbf{D}_{\beta}^{l} V(\Omega)\right\|=\left(\left\|\tilde{u} ; \mathscr{D}_{\beta}^{l} V(\Omega)\right\|+\sum_{j=1}^{J}\left\|k_{j} ; H^{l-\beta}(\Upsilon)\right\|^{2}\right)^{1 / 2}
$$

Так как операторы $\left\{\mathscr{L}^{0}\left(\nabla_{\eta}, 0\right), \mathscr{B}^{0}\left(\eta, \nabla_{\eta}, 0\right)\right\}$ модельных задач в $\mathbb{K}^{0}$ аннулируют полиномы $\eta \mapsto p^{(j)}(\eta, z)$, свойства оператора продолжения $\mathfrak{C}$ обеспечивают непрерывность отображения

$$
\mathbf{A}_{\beta}^{l}=\{\mathscr{L}, \mathscr{B}\}: \mathbf{D}_{\beta}^{l} V(\Omega) \rightarrow \mathscr{R}_{\beta}^{l} V(\Omega)
$$

где $\mathscr{R}_{\beta}^{l} V(\Omega)$ - то же пространство (2.6), что и в $(2.7)$.

ТЕОРема 2.17. Пусть полиномиальное свойство $1^{0}$ у пары $\{\mathscr{L}$, a $\}$ сохраняется при замораживании коэффициентов в $\bar{\Omega}$, и при $x^{0} \in \Upsilon$ форма $a^{0}$, обслуживающая модельную задачу в $\mathbb{K}\left(x^{0}\right)$, удовлетворяет $3^{0}$. При указанных ограничениях на подпространства $P\left(x^{0}\right)$ существует такое $\delta>0$, что в случае $l \geqslant \max \mathbf{t} u$ $\beta \in(l-\delta, l)$ оператор (2.47) задачи (2.1), (2.2) фредгольмов, а его ядро и коядро находятся по формулам (2.20) и (2.21), в которых вместо $\mathscr{P}+(\mathscr{B})$ фигурирует линеал (2.36).

Так как коэффишиенты $k_{1}, \ldots, k_{J}$ не имеют даже первых производных, простое продолжение $\mathscr{V} \ni(y, z) \mapsto \chi\left(h^{-1}|y|\right) k_{j}(z)$ множителей $k_{j}$ с ребра в $\Omega$ лишает получаемьй остаток $\tilde{u}$ требуемой гладкости в $\Omega$, т.е. без участия оператора $\mathfrak{C}$ ввести норму $(2.46)$ не удается. Разумеется, при $d=0$ оператор продолжения не нужен и после естественных упрошений теоремы 2.17 и 2.13 доставляют фредгольмов оператор с нулевьм индексом для задачи (2.1), (2.2) в области с коническими точками.

Техника отделения асимптотики, описанная здесь в простейшем варианте, оказывается полезной во многих вопросах. В [64], [65], [68]-[70] с ее помощью осуществлялись постановки (асимптотических) условий на подмногообразиях границы, описьвались самосопряженные расширения дифференциальных операторов и строились псевдодифференциальные операторы на ребрах, порожденные эллиптическими задачами 
и обобщающие такие классические характеристики задач, как матрищы рассеяния и поляризации. В рамках такого подхода ясную интерпретацию приобретают метод срашиваемых асимптотических разложений (см. [71]) и критерии разрушения, учитываюшие эффекты, локализованные в устьях трешин (см. [72]). Наконец, введение неизвестных угловых частей степенных решений (в (2.16) они вполне определены модельной задачей $\left\{\mathscr{L}^{0}, \mathscr{B}^{0}\right\}$ в $\mathbb{K}^{0}$ ) позволяет исследовать асимптотику решений даже тех краевых задач для нелинейных уравнений, которые не допускают линеаризацию из-за совпадения асимптотических порядков у линейных и нелинейных членов (см. [73] и др.).

5. Область с пересекающимися ребрами (многогранными углами).

Пусть множество $\Upsilon$ нерегулярностей границы $\partial \Omega$ является $d$-мерным подмногообразием, гладким всюду, кроме начала координат $O$, а в окрестности $\mathscr{V} \ni O$ область $\Omega$ совпадает с конусом $\mathbb{K}$, вырезаюшим на сфере $\mathbb{S}^{n-1}=\{x: r \equiv|x|=1\}$ область $\omega$. У $\omega$ имеется свое нерегулярное множество $v=\Upsilon \cap \mathbb{S}^{n-1}$, оказываюшееся гладким $(d-1)$-мерным подмногообразием гранищы $\partial \omega$. Если $n=3$ и $d=1$, то точка 0 может быть вершиной многогранного угла, а $\omega$ - сферическим многоугольником с вершинами $\varphi^{1}, \ldots, \varphi^{M} \subset v$.

В области $\Omega$ рассмотрим задачу $(2.1),(2.2)$ с краевьми условиями Неймана, т.е. возьмем оператор $\mathscr{B}=\mathscr{N}$ из формулы Грина (1.2). В согласии с (2.8) и (2.9) главные (в точке $O$ ) части $\mathscr{L}^{0}$ и $\mathscr{N}^{0}$ операторов $\mathscr{L}$ и $\mathscr{N}$ запишем при помоши сферических координат $(r, \varphi)$ и введем пучок

$$
\mathfrak{A}(\lambda)=\left\{L^{0}\left(\varphi, \partial_{\varphi}, \lambda\right), N^{0}\left(\varphi, \partial_{\varphi}, \lambda\right)\right\}
$$

которому предназначена та же роль, что и пучку (2.10) в случае вершин конусов с гладкой направляющей. В обсуждаемой задаче особенности границы $\partial \omega$ осложняют поиск подходящей области определения пучка (2.48). Теперь становятся понятньми наши старания обеспечить нулевой индекс у операторов краевых задач в областях с гладкими ребрами - пучок (2.48) должен осушествлять изоморфизм при всех $\lambda \in \mathbb{C}$, за исключением счетного набора изолированных точек из множества (2.11). В [27] показано, что в некритическом случае полиномиальное свойство $3^{0}$ гарантирует справедливость постулатов общей теории [23], а в критическом случае $(n-d-$ четное число, $n-d \leqslant 2 \max \mathbf{t})$, когда теория [23] не работает, позволяет подобрать нужную область определения. Здесь мы ограничимся констатацией фактов, относящихся к критическому случаю (остальное можно найти в [27] и [23]).

Пространства $V_{\beta}^{l}(\omega, v)$ и $V_{\beta}^{l, s}(\omega, v)$ функций на $\omega$ определяются в соответствии с формулами (2.3) и (2.38), в которых градиент $\nabla_{x}$ заменен его сферической частью $\nabla_{\varphi}$, а расстояние $\rho$ до ребра $v$ измеряется на сфере $\mathbb{S}^{n-1}$. Векторные пространства $\mathscr{D}_{\beta}^{l,(d-n) / 2} V(\omega, v)$ и $\mathscr{R}_{\beta}^{l} V(\omega, v)$ составляются по прежним правилам.

ПРЕДЛОЖЕНИЕ 2.18 ([27]). Пусть $n-d$ - четное число, не превосходящее $2 \max \mathbf{t}$, а форма $a^{0}$, получающаяся из (1.3) замораживанием коэффичиентов в точке $O$, обладает полиномиальным свойством $3^{0}$. Тогда при $l \geqslant \max \mathbf{t} u$ $\beta \in(l-\delta, l)$ с некоторым $\delta>0$ отображение

$$
\mathfrak{A}(\lambda): \mathscr{D}_{\beta}^{l,(d-n) / 2} V(\omega, v) \rightarrow \mathscr{R}_{\beta}^{l} V(\omega, v)
$$


оказивается изоморфизмом при всех $\lambda \in \mathbb{C}$, лежсащих вне замкнутого двойного угла

$$
\{\mu \in \mathbb{C}:|2 \operatorname{Re} \mu+n| \leqslant \gamma|\operatorname{Im} \mu|\}, \quad \gamma>0 .
$$

В таком угле расположсено счетное множсество нормальных собственных чисел, сгущаюшееся лишь на бесконечности, а вершина $\lambda_{0}=-n / 2$ попадает в это мно сество лишь в том случае, если существует нетривиальный однородный полином $p=\left(p_{1}, \ldots, p_{T}\right)$ обобщенной степени $-n / 2$, удовлетворяющий условиям

$$
\left.\nabla_{x}^{s} p_{j}\right|_{v}=0, \quad s<t_{j}-n / 2, \quad j=1, \ldots, T .
$$

Полная алгебраическая кратность собственного числа $\lambda_{0}$ совпадает с удвоенной размерностью линеала $P_{0}^{0}$ названньх полиномов, $\operatorname{ker} \mathfrak{A}\left(\lambda_{0}\right)=\left.P_{0}^{0}\right|_{\omega} u$ каждый собственный вектор имеет присоединенный.

Как обычно, отсутствие на прямой $i \mathbb{R}-\left(\beta^{0}-l+n / 2\right)$ собственных чисел пучка $(2.49)$ позволяет применить преобразование Меллина (см. замечание $2.2(1))$ и при помощи равномерных относительно параметра $\lambda \in i \mathbb{R}-\left(\beta^{0}-l+n / 2\right)$ оценок резольвенты $\mathfrak{A}(\lambda)^{-1}$ (подробности см. в $[27 ; \S 3]$ ) установить однозначную разрешимость модельной задачи $(2.12)$ в пространстве $\mathscr{D}_{\beta, \beta^{0}}^{l,(d-n) / 2} V(\mathbb{K}, v, O)$ с нормой

$$
\begin{aligned}
& \left(\sum_{k=1}^{T} \sum_{q=0}^{l+t_{k}} \int_{0}^{\infty} r^{2\left(\beta^{0}-l-t_{k}\right)+n-1}\right. \\
& \left.\quad \times\left\|\left(r \frac{\partial}{\partial r}\right)^{q} u_{k}(r, \cdot) ; V_{\beta}^{l+t_{k}-q, t_{k}-q-(n-d) / 2}(\omega, v)\right\|^{2} d r\right)^{1 / 2},
\end{aligned}
$$

порожденной весовой нормой в пространстве $\mathscr{D}_{\beta}^{l,(d-n) / 2} V(\omega, v)$ на сечении $\omega$ конуса $\mathbb{K}$ с негладкой направляющей. В области $\Omega$ также вводятся два весовых множителя расстояние $\rho$ до множества $\Upsilon \subset \partial \Omega$ и расстояние $r$ до точки $O$. Именно, пространство $V_{\beta, \beta^{0}}^{l, s, s^{0}}(\Omega, \Upsilon, O)$ является пополнением $C_{0}^{\infty}(\bar{\Omega} \backslash \Upsilon)$ по весовой норме со ступеньками на уровнях $s$ и $s^{0}$

$$
\left(\sum_{q=0}^{l}\left\|r^{\beta^{0}-\beta-\Theta(s-q)+\Theta\left(s^{0}-q\right)} \rho^{\beta-l+q+\Theta(s-q)} \nabla_{x}^{q} w ; L_{2}(\Omega)\right\|^{2}\right)^{1 / 2}
$$

Уничтожая ступеньки, т.е. вычеркивая функции Хевисайда $\Theta(s-q)$ и $\Theta(s-q), \Theta\left(s^{0}-\right.$ $q)$, приходим к классам $V_{\beta, \beta^{0}}^{l, s}(\Omega, \Upsilon, O)$ и $V_{\beta, \beta^{0}}^{l}(\Omega, \Upsilon, O)$ соответственно. Как и ранее, определяются следовые и векторные пространства (ср. с (2.4)-(2.6)). Отметим, что норму

$$
\left\|\cdot ; \mathscr{D}_{\beta, \beta^{0}}^{l,(d-n) / 2} V(\Omega, \Upsilon, O)\right\|
$$

можно получить “склеиванием” норм

$$
\left\|\cdot ; \mathscr{D}_{\beta}^{l,(d-n) / 2} V(\Omega, \Upsilon)\right\| \text { и }\left\|\cdot ; \mathscr{D}_{\beta, \beta^{0}}^{l,(d-n) / 2} V(\mathbb{K}, v, O)\right\|,
$$

фигурировавших в $(2.38)$ и (2.50). 
TeOpema 2.19 ([27]). Пусть $n-d$ есть четное число, $n-d \leqslant 2 \max \mathbf{t}$, а форма (1.3), отвечающая задаче Неймана (2.1), (2.2), обладает полиномиальным свойством $3^{0}$, выдерживающим заморажсивние во всякой точке замыкания $\bar{\Omega}$. Тогда найдутся такие положительнье числа $\delta$ и $\delta^{0}$, что при $l \geqslant \max \mathbf{t} u$ $\beta \in(l-\delta, l)$ выполняются следующие утверждения.

1) Отображсение

$$
\{\mathscr{L}, \mathcal{N}\}: \mathscr{D}_{\beta, \beta^{0}}^{l,(d-n) / 2} V(\Omega, \Upsilon, O) \rightarrow \mathscr{R}_{\beta, \beta^{0}}^{l} V(\Omega, \Upsilon, O)
$$

является фредгольмовым в том и только том случае, если прямая $i \mathbb{R}-\left(\beta^{0}-\right.$ $l+n / 2)$ свободна от спектра пучка (2.49).

2) Если линеал $P_{0}^{0}$, указанный в предложсении 2.18, тривиален, то при $\mid \beta^{0}-$ $l<\delta^{0}$ оператор (2.51) задачи Неймана (2.1), (2.2) имеет нулевой индекс и его ядро совпадает с подпространством $\mathscr{P}_{*}^{+}$полиномов из $\mathscr{P}_{*}(\mathscr{N})($ см. $(2.36))$, подчиненных условиям (2.25) с $x^{0}=O$ и $M=1$. Условиями разрешимости задачи служат равенства

$$
(f, p)_{\Omega}+(g, \mathscr{D} p)_{\partial \Omega}=0 \quad \forall p \in \mathscr{P}_{*}^{+}
$$

3) Ecли $\operatorname{dim} P_{0}^{0}=0$, то при $\beta^{0} \in\left(l-\delta^{0}, l\right)$ оператор задачи (2.1), (2.2),

$$
\{\mathscr{L}, \mathscr{N}\}: \mathscr{D}_{\beta, \beta^{0}}^{l,(d-n) / 2,-n / 2} V(\Omega, \Upsilon, O) \rightarrow \mathscr{R}_{\beta, \beta^{0}}^{l} V(\Omega, \Upsilon, O)
$$

фредгольмов, причем его индекс равен нулю, ядро совпадает с линеалом полиномов $\mathscr{P}_{*}^{+}$, а коядро принимает вид $\left\{(p, \mathscr{D} p): p \in \mathscr{P}_{*}^{+}\right\}$(cм. (2.52)).

ЗАмЕчАниЕ 2.20. В условиях теорем 2.13 и $2.19(2,3)$ для операторов простой структуры $\mathscr{P}(\mathscr{N})=\mathscr{P}$ в силу предложения 1.5. Поэтому ядра указанных в теоремах операторов задачи Неймана состоят из тех полиномов $p \in \mathscr{P}$, которые принадлежат областям определения операторов. Переформулировка последнего включения как раз и приводит к условиям Соболева (2.36) на $\Upsilon$ или (2.25) в точке $O$. Для систем уравнений второго порядка (примеры 1.12 и 1.13) $t_{j}=1$ и $t_{j}-(n-d) / 2 \leqslant 0$, т.е. условия Соболева исчезают и ядра совпадают с полньм линеалом $\mathscr{P}$.

Общая схема [23] исследования геометрических особенностей более сложной структуры порождает иерархию модельных и эталонных задач (ср. с (2.12), (2.28) и (2.27) соответственно). Приемы, описанные в данном параграфе, позволяют последовательно рассмотреть эти задачи и реализовать схему в случае краевых условий Дирихле или Неймана при наличии полиномиального свойства у системы дифференциальных уравнений в области (подробности см. в [27]). Если подобные $P_{0}^{0}$ линеалы полиномов оказываются тривиальньми (см. (2.33) и предложение 2.18), то и смешанные краевые задачи обрабатываются по схеме из [23] (ср. с замечанием 2.15). При нетривиальных линеалах типа $P_{0}^{0}$ требуются пространства с отделенной асимптотикой (п. $\left.4 \S 2\right)$ или комбинации ступенчатых и обычных весовых норм (пример 2.14), однако смешанные краевые задачи изучались в [64], [65] только для областей с гладкими ребрами.

6. О собственных числах пучков. В большинстве утверждений данного параграффа устанавливались границы изменения весовых индексов, которые согласно [13], [14], [22], [23], [27] определяются спектром пучков вида (2.10) и (2.49), (2.48). Для 
конкретных задач математической физики проделано множество расчетов таких собственных чисел (см. [74], [62], [41], [75], [63], а также упомянутые там ссылки); в некоторых случаях для них найдены асимптотические формулы (см. [76]-[79], [43; гл. 10] и др.). Кроме того, в [80]-[82] установлены тонкие оценки размеров полос, заведомо свободных от спектра пучков, порожденных операторами Ламе и Стокса (т.е. для величин $\delta$ и $\delta_{0}$, возникавших в теоремах этого параграфа).

Полное описание спектра пучков для достаточно общих систем уравнений возможно лишш при простейших формах конусов - проколотого пространства $\mathbb{R}^{n} \backslash O$ и полупространства $\mathbb{R}_{+}^{n}$ (см. [83]), а также угла с раствором $2 \pi$ (см. [84]). Именно такие формы возникают в асимптотических методах при сингулярном возмушении гладких границ ([42], [43], [71], [85] и др.), в теории задач Соболева ([61], [31; § 12.7] и др.) и в механике разрушения ([72], [31; гл. 7] и др.). Сформулируем несколько утверждений относительно оператор-матрищ (1.10) простой структуры с краевыми условиями, соответствующими (1.6); при этом особое внимание уделяем задачам Дирихле и Неймана.

ПРЕДЛОЖЕНИЕ 2.21 ([83], [71]). 1) Если $\mathbb{K}=\mathbb{R}^{n} \backslash O$, то спектр пучка $(2.10)$,

$$
\mathbb{C} \ni \lambda \mapsto \mathfrak{A}(\lambda)=L\left(\varphi, \partial_{\varphi}, \lambda\right): H^{l+\mathbf{t}}\left(\mathbb{S}^{n-1}\right) \rightarrow H^{l-\mathbf{t}}\left(\mathbb{S}^{n-1}\right),
$$

совпадает с обвединением множеств $\mathbb{Z}_{+}(-\max \mathbf{t}) u \mathbb{Z}_{-}(n-\max \mathbf{t})$, əде

$$
\mathbb{Z}_{ \pm}(k)=\{q \in \mathbb{Z}: \pm q \geqslant k\}, \quad \mathbb{Z}=\{0, \pm 1, \pm 2, \ldots\}
$$

Степенные решения (2.16) являются либо полиномами, либо линейными комбинациями производных столбцов фундаментальной матрицы для оператора $\mathscr{L}^{0}\left(\nabla_{x}\right)$ в $\mathbb{R}^{n}$.

2) всли $\mathbb{K}=\mathbb{R}_{+}^{n}$, то спектр пучка $(2.10)$ содерэсится в обвединении $\mathbb{Z}_{+}(-\max \mathbf{t})$ $\cup \mathbb{Z}_{-}(n-\max \mathbf{t})$. В случае условий Неймана он не отличается от этого обтединения, а в случае условий Дирихле равен $\mathbb{Z}_{+}(0) \cup \mathbb{Z}_{-}(n)$. Степенные решения (2.16) являются либо полиномами, либо производными в касательных направлениях ядер Пуассона для задачи $\left\{\mathscr{L}^{0}\left(\nabla_{x}\right), \mathscr{B}^{0}\left(\nabla_{x}\right)\right\}$ в $\mathbb{R}_{+}^{n}$.

В очередном утверждении $n=2$ и $\mathbb{K}$ - плоскость с полубесконечным разрезом,

$$
\mathbb{K}=\mathbb{R}^{2} \backslash\left\{x: x_{1} \leqslant 0, x_{2}=0\right\}=\{x=(\rho \cos \varphi, \rho \sin \varphi): \rho>0,|\varphi|<\pi\} .
$$

ПРЕДЛОЖЕНИЕ 2.22 ([84], [86]). 1) Спектр пучка (2.10), отвечающего задаче Дирихле для оператора $\mathscr{L}^{0}\left(\nabla_{x}\right)$ в $\mathbb{K}$, совпадает с множеством

$$
\mathscr{Z}_{\mathscr{D}} \cup\left\{\lambda: \lambda=i \mu_{q}+k+1 / 2, k \in \mathbb{Z}, q=1, \ldots, Q\right\}
$$

где $\mathscr{Z}_{\mathscr{D}}=\mathbb{Z} \backslash\{-1\}$, а $Q$ и $\mu_{1}, \ldots, \mu_{q}$ - натуральное и вещественные числа (не зависящие от $k$ ). Все собственные числа просты алгебраически, т.е. присоединенных векторов нет. Полная кратность собственных чисел на прямой $i \mathbb{R}+k+1 / 2$ равна $m=t_{1}+\cdots+t_{T} ;$ размерности собственньх подпространств 
$\operatorname{ker} \mathfrak{A}\left(i \mu_{q}+k+1 / 2\right)$ одинаковы при всех $k \in \mathbb{Z}$. Кратность собственньх чисел $p \in\{0,1,2, \ldots\}$ равна

$$
\sum_{j=1}^{T} \min \left\{p+1, t_{j}\right\}
$$

а отвечающие им степенные решения оказываются полиномами. Ту же кратность (2.56) имеют собственнье числа -2-p.

2) Спектр пучка (2.10), отвечающего задаче Неймана для оператора $\mathscr{L}^{0}\left(\nabla_{x}\right)$ в $\mathbb{K}$, совпадает с множсеством

$$
\mathscr{Z}_{\mathcal{N}} \cup\left\{\lambda: \lambda=i \mu_{q}+k+1 / 2, k \in \mathbb{Z}, q=1, \ldots, Q\right\},
$$

где $\mathscr{Z}_{\mathcal{N}}=\mathbb{Z}$, а остальные обозначения - те жсе, что и в (2.55). Простыми являются все собственные числа, кроме чельх точек отрезка $I=[-\max \mathbf{t}, \max \mathbf{t}-2]$, частные кратности которых не превосходят двух. Полная кратность собственных чисел на прямой $i \mathbb{R}+k+1 / 2$ равна $m=t_{1}+\cdots+t_{T} ;$ размерности подпространств $\operatorname{ker} \mathfrak{A}\left(i \mu_{q}+k+1 / 2\right)$ одинаковь при всех $k \in \mathbb{Z}$. Если $p \in \mathbb{Z}$, то сумма

$$
s(p)=\sum_{j=1}^{T} \min \left\{\left(p+1+t_{j}\right)_{+}, t_{j}\right\}
$$

указывает размерность подпространства в $\operatorname{ker} \mathfrak{A}(p)$, состоящего из следов на окружности $\mathbb{S}^{1}$ полиномов переменных $x=\left(x_{1}, x_{2}\right)$. Полные алгебраические кратности собственных чисел $p u-2-p$ равны $s(p)+s(-2-p)$. Наконеи, $\operatorname{dim} \operatorname{ker} \mathfrak{A}(p)=s(p)$ при $p \geqslant-1$, т.е. для таких $р$ все собственные векторы следы полиномов и существует $s(-2-p)$ присоединенных векторов первого порядка.

ЗАмЕчАниЕ 2.23. 1) Согласно [84] для справедливости предложения 2.22 необходима монотонная зависимость энергии от длины трешины. В задачах Дирихле и Неймана энергетический функционал, разумеется, возрастает и убывает соответственно, однако для смешанных краевых задач монотонности нет. Например, степенные решения уравнения Лапласа $-\Delta_{x} u=0$ в $\mathbb{K}$ с условиями Дирихле при $\varphi=-\pi$ и Неймана при $\varphi=\pi$ имеют вид $r^{\lambda} \sin \lambda(\varphi+\pi)$, где $\lambda=k \pm 1 / 4$ и $k \in \mathbb{Z}$, т.е. спектр пучка не обладает провозглашенными свойствами. Из-за "неправильного" знака при блоке $Q_{22}$ матрищы $Q$ из (1.40) задачи Дирихле и Неймана для системы уравнений теории пезоэлектрических тел не подчиняются предложению 2.22 , однако основными в теории являются смешанные условия

$$
\begin{gathered}
\Lambda^{\prime}(\nu(x))^{t} Q \Lambda\left(\nabla_{x}\right) u(x)=g(x), \quad x \in \partial \Omega, \\
u_{4}(x)=0, \quad x \in \partial \Omega,
\end{gathered}
$$

означаюшие, что механические усилия $g$ приложены к поверхности $\partial \Omega$, электропроводящей и в силу $(2.59)$ эквипотенциальной $\left(\right.$ в $(2.58)(9 \times 3)$-матрица $\Lambda^{\prime}(\xi)$ составлена 
из трех первых столбцов матрицы $\Lambda(\xi)$; см. (1.40)). Соответствующий энергетический функционал убывает при росте трещины, на берегах которой назначены условия (2.58), (2.59), а значит, предложение 2.22 сохраняет силу.

2) В [84] спектры были погружены в множество (2.55) или (2.57); кроме того, была проверена алгебраическая простота собственных чисел с полуцелыми вешественными частями. Остальные заключения об их количестве и кратностях делаются на основе предложения 2.3 с привлечением трюка [86]: поскольку собственные числа не могут покидать прямые $i \mathbb{R}+k+1 / 2$ или множество $\mathbb{Z}$, в силу [87] их кратности такие же, как и у пучка, порожденного диагональной матрицей $\operatorname{diag}\left\{\Delta_{x}^{t_{1}}, \ldots, \Delta_{x}^{t_{T}}\right\}$, к которой можно придти непрерывным искажением оператора $\mathscr{L}^{0}\left(\nabla_{x}\right)$. В $[84]$ показано также, что предложение 2.22 приспосабливается к задачам с кусочно постоянньми коэффициентами - на луче $\left\{x: x_{1}>0, x_{2}=0\right\}$ допускается постановка условий сопряжения (трансмиссии). Подсчет кратностей собственных чисел из предложения 2.21 нетрудно произвести посредством приема из [88; § 13.1] (см. [83], [85]).

В задачах механики разрушения и других приложениях асимптотических методов важную роль играет нормировка степенных решений (см. [15] и [20; 5.2$],[31 ; \S 6.1]$ ). Для полного конуса $\mathbb{K}=\mathbb{R}^{n} \backslash O$ все условия нормировки и биортогональности достигаются при помоши алгебраических операций и дифференцирования (см. [31; §6.4] и [71], [85]); та же процедура пригодна в случае $\mathbb{K}=\mathbb{R}_{+}^{n}$. Для трешины к упрошениям приводят следуюшие два наблюдения [84], [72]. Во-первых, производная $\partial \mathscr{U} / \partial x_{1}$ решения (2.17) задачи в угле (2.54) оказывается степенным решением с показателем $\lambda-1$, которое аннулируется лишь при $\mathscr{U}=\mathscr{U}\left(x_{2}\right)$ (поэтому степенное решение, отвечаюшее собственному числу $\lambda=0$ из $(2.55),-$ полином переменной $\left.x_{2}\right)$. Во-вторых, в антисимметричной форме

$$
q(\mathscr{U}, \mathscr{V})=(\mathscr{N} \mathscr{U}, \mathscr{D} \mathscr{V})_{\Gamma}-(\mathscr{D} \mathscr{U}, \mathscr{N} \mathscr{V})_{\Gamma},
$$

проистекающей из (1.2) и отвечаюшей за обсуждаемую ортогонализацию, возможна "переброска" производных

$$
q\left(\partial \mathscr{U} / \partial x_{1}, \mathscr{V}\right)=-q\left(\mathscr{U}, \partial \mathscr{V} / \partial x_{1}\right)
$$

(см. [84], [85]); здесь $Г$ - произвольная дуга, соединяющая берега разреза внутри угла (2.54), а сама формула (2.60) верна лишь для степенных решений $\mathscr{U}$ и $\mathscr{V}$. Таким образом, достаточно соблюсти условия биортогональности для решений, отвечаюших собственным числам на прямых $i \mathbb{R}-1 \pm 1 / 2,-$ формула (2.60) распространит их на все элементы базиса, достроенные при помощи интегрирования и дифференцирования вдоль трещины (по переменной $x_{1}$ ).

7. Задачи Соболева. В этом разделе показывается, как предшествуюшие результаты приспосабливаются к задачам с данньми на внутренних подмногообразиях. Поскольку формулировки теорем практически не изменяются, ограничимся рассмотрением примеров, связанных с уравнениями четвертого порядка.

ПримеР 2.24. Рассмотрим задачу Неймана для системы теории пластин, описанной в примере 1.14. Точки $x^{1}, \ldots, x^{M}$ могут быть как угловыми, так и внутренними 
(если все они лежат в $\Omega$, то граница $\partial \Omega$ гладкая). Согласно (1.42) линеалы полиномов (2.20) и (2.15), (2.24) вьглядят так:

$$
\begin{aligned}
& \mathscr{P}_{+}(\mathscr{N})=\left\{\left(-c_{0} x_{2}, c_{0} x_{1}, 0\right): c_{0} \in \mathbb{R}\right\} \\
& \mathscr{P}_{0}^{\mu}(\mathscr{N})=\left\{\left(c_{1}, c_{2}, C_{1}\left(x_{1}-x_{1}^{\mu}\right)+C_{2}\left(x_{2}-x_{2}^{\mu}\right)\right): c_{i}, C_{i} \in \mathbb{R}, i=1,2\right\}, \\
& \mathscr{P}_{-}(\mathscr{N})=\left\{\left(0,0, C_{0}\right): C_{0} \in \mathbb{R}\right\} .
\end{aligned}
$$

По теореме 2.13 оператор задачи $\mathscr{A}_{\beta}^{l,-1}$ с областью определения

$$
\mathscr{D}_{\beta}^{l,-1} V(\Omega)=V_{\beta}^{l+1,0}(\Omega)^{2} \times V_{\beta}^{l+2,1}(\Omega), \quad l \in\{2,3, \ldots\}, \quad \beta \in(l-\delta, l),
$$

фредгольмов с нулевым индексом, а его ядро и коядро описываются при помощи линеала $\mathscr{P}_{*}(\mathscr{N})$ из $(2.36)$. В случае $M=1$ (множество иррегулярностей $\Upsilon$ состоит из одной точки $x^{1}$ ) получаем

$$
\mathscr{P}_{*}(\mathscr{N})=\left\{\left(c_{1}-c_{0} x_{2}, c_{2}+c_{0} x_{1}, C_{1}\left(x_{1}-x_{1}^{1}\right)+C_{2}\left(x_{2}-x_{2}^{1}\right)\right): c_{q}, C_{i} \in \mathbb{R}\right\}
$$

Если точки $x^{1}, \ldots, x^{M}$ лежат на прямой $\mathbb{L}=\left\{x: a_{1} x_{1}+a_{2} x_{2}=a_{0}\right\}$, то в $(2.62)$ коэффициенты $C_{1}$ и $C_{2}$ связьваются соотношением $a_{2} C_{1}=a_{1} C_{2}$, т.е. размерность линеала уменьшается до четырех. Наконец, при наличии невырожденного треугольника с вершинами из $\Upsilon$ в $(2.62) C_{1}=C_{2}=0$ и $\operatorname{dim} \mathscr{P}_{*}(\mathscr{N})=3$. Влияние геометрии на подпространства ker $\mathscr{A}_{\beta}^{l,-1}$ и coker $\mathscr{A}_{\beta}^{l,-1}$ обусловлено постановкой условий Соболева $u_{3}(x)=0, x \in \Upsilon$, означающих жесткое защемление пластины в точках $x^{1}, \ldots, x^{M}$ и оказывающихся корректными в силу вложений $V_{\beta}^{l+2,1}(\Omega) \subset H^{2}(\Omega)($ см. $(2.38)$ и $(2.62))$ и $H^{2}(\Omega) \subset C(\bar{\Omega})$.

Рассмотрим задачу Дирихле для бигармонического уравнения

$$
\Delta^{2} u(x)=f(x), x \in \Omega ; u(x)=\partial_{\nu} u(x)=0, x \in \partial \Omega
$$

и соответствуюшую задачу Соболева

$(2.64) \Delta^{2} w(x)=f(x), x \in \Omega \backslash \Upsilon ; \quad w(x)=\partial_{\nu} w(x)=0, x \in \partial \Omega ; \quad w(x)=0, x \in \Upsilon$,

где $\Omega$ - область с гладкой границей и $\Upsilon \subset \Omega$. Пусть сначала $\Omega \subset \mathbb{R}^{2}$ и для простоты $\Upsilon=\{O\}$, т.е. обе задачи связываются с теорией пластин Кирхгофа (см. далее пример $4.6(2))$. Если $f \in L_{2}(\Omega)$, то обобшенные постановки задач (2.63) и (2.64) приводят к их однозначной разрешимости соответственно на подпространствах

$$
\begin{gathered}
\stackrel{\circ}{H}^{2}(\Omega)=\left\{u \in H^{2}(\Omega): u=\partial_{\nu} u=0 \text { на } \partial \Omega\right\}, \\
\stackrel{\circ}{H}(\Omega, O)=\left\{w \in \stackrel{\circ}{H}^{2}(\Omega): w(O)=0\right\} .
\end{gathered}
$$

Связь решений $u$ и $w$ поясняется в следующих утверждениях. 
Лемма 2.25. 1) Функиия Грина $G(\cdot, y)$ задачи (2.63) с особенностью в точке $y \in \Omega$ попадает в пространство $\stackrel{\circ}{H}^{2}(\Omega)$ и допускает представление

$$
G(x, y)=G^{0}(x, y)-(8 \pi)^{-1}|x-y|^{2} \ln |x-y|,
$$

причем регулярная часть $G^{0}$ гладко зависит от $x \in \bar{\Omega} u$

$$
G^{0}(y, y)=G(y, y)=\int_{\Omega}\left|\Delta_{x} G(x, y)\right|^{2} d x>0 .
$$

2) Решения $u \in \stackrel{\circ}{H}^{2}(\Omega)$ и $w \in \stackrel{\circ}{H}^{2}(\Omega, O)$ задач (2.63) и (2.64) связаны равенством

$$
w(x)=u(x)-G(O, O)^{-1} u(O) G(x, O) .
$$

ПРЕДЛОЖЕНИЕ 2.26 ([89]). 1) Неограниченный оператор $\mathbf{A}_{0}$ в $L_{2}(\Omega)$, заданный дифференииальным выражсением $\Delta^{2}$ и имеющий область определения

$$
\mathscr{D}\left(\mathbf{A}_{0}\right)=\left\{u \in H^{4}(\Omega) \cap \stackrel{\circ}{H^{2}}(\Omega): \nabla_{x}^{s} u(0)=0, s=0,1,2\right\},
$$

является замкнутым и симметрическим, а область определения сопряжсенного оператора $\mathbf{A}_{0}^{*}=\Delta^{2}$ такова:

$$
\begin{aligned}
\mathscr{D}\left(\mathbf{A}_{0}^{*}\right)=\left\{u: u=u^{0}+\sum_{k=0}^{5}\left(a_{k} \chi V_{k}+b_{k} G_{k}\right),\right. \\
\left.u^{0} \in \mathscr{D}\left(\mathbf{A}_{0}\right), a=\left(a_{0}, \ldots, a_{5}\right)^{t}, b=\left(b_{0}, \ldots, b_{5}\right)^{t} \in \mathbb{R}^{6}\right\} ;
\end{aligned}
$$

здесь $\chi \in C_{0}^{\infty}(\Omega), \chi=1$ в окрестности точки $O$,

$$
\begin{gathered}
V_{0}=1, \quad V_{i}(x)=x_{i}, \quad V_{2+i}(x)=2^{-1 / 2} x_{i}^{2} \quad(i=1,2), \quad V_{5}(x)=x_{1} x_{2}, \\
G_{k}(x)=\left.V_{k}\left(\nabla_{y}\right) G(x, y)\right|_{y=0} .
\end{gathered}
$$

2) Пусть $\mathbb{R}^{6}=\mathbf{N}^{+} \oplus \mathbf{N}^{0} \oplus \mathbf{N}^{-}$- ортогональное разложсение и $\mathbf{S}$ - симметрический обратимый оператор в $\mathbf{A}^{0}$. Сужение $\mathbf{A}$ оператора $\mathbf{A}_{0}^{*}$ на линеал

$$
\mathscr{D}(\mathbf{A})=\left\{u \in \mathscr{D}\left(\mathbf{A}_{0}^{*}\right): a=\mathbf{n}^{+}+\mathbf{n}^{0}, b=\mathbf{n}^{-}+\mathbf{S n}^{0}, \mathbf{n}^{\theta} \in \mathbf{N}^{\theta}, \theta=0, \pm\right\}
$$

оказывается самосопряженным расширением оператора $\mathbf{A}_{0}$. Всякое самосопрязсенное расширение описывается указанным способом.

3) Расширение по Фридрихсу (m.е. $\left.\mathbf{N}^{+}=\mathbb{R}^{6}, \mathbf{N}^{-}=\mathbf{N}^{0}=\{0\}^{6}\right)$ отвечает задаче (2.63), а расширение, в котором $\mathbf{N}^{+}=\{0\} \times \mathbb{R}^{5}, \mathbf{N}^{-}=\mathbb{R} \times\{0\}^{5} u \mathbf{N}^{0}=\{0\}^{6}$, - задаче Соболева (2.64).

По поводу техники самосопряженных расширений сошлемся на основополагающую работу [90], обзор [91] и статьи [68], [71], [85]. В последних рассматриваются расширения симметрических операторов в весовых классах и с помощью методов, разработанных в [40], [43], дается асимптотическое истолкование моделям сред с точечньми дефектами (см. [91]-[93] и др.) и вьводятся оценки их погрешностей. 
Обсудим многомерную задачу Соболева (2.64). Если $\Upsilon$ - гладкий замкнутьй контур внутри $\Omega \subset \mathbb{R}^{3}$, то задача обслуживается теоремой 2.13 : оператор с областью определения $V_{\beta}^{l+2,1}(\Omega)$ является изоморфизмом при $l \geqslant 2$ и $\beta \in(l-1, l)$. Если же $\Upsilon$ - гладкая дуга с конщами $x^{ \pm}$, то нужный результат доставляет теорема 2.19. В качестве области определения берется пространство $V_{\beta, \beta^{0}}^{l+2,1}\left(\Omega, \Upsilon, x^{ \pm}\right)$, а оператор задачи (2.64) оказывается изоморфизмом в случае $l \geqslant 2, \beta \in(l-1, l)$ и $\left|\beta^{0}-l\right|<1 / 2$. Конкретные значения числам $\delta$ и $\delta^{0}$, фигурируюшим в использованных теоремах и задающим границы изменения весовых индексов, придают предложение 2.21 (1) и

ПРЕДЛОЖЕНИЕ 2.27. Пусть $\mathscr{L}$ - оператор-матрица (1.10). Собственные числа $\lambda$ пучка (2.49), порожсенного модельной задачей

$$
\begin{gathered}
\mathscr{L}^{0}\left(\nabla_{x}\right) u(x)=0, \quad x \in \mathbb{K}=\mathbb{R}^{n} \backslash \overline{\mathbb{L}_{+}}, \\
\nabla_{x}^{s} u_{j}(x)=0, \quad x \in \mathbb{L}_{+}=\left\{x: x_{1}=\cdots=x_{n-1}=0, x_{n}>0\right\}, \\
s<t_{j}-n / 2, \quad j=1, \ldots, T,
\end{gathered}
$$

удовлетворяют включениям $\operatorname{Re} \lambda \in \mathbb{Z}$ при четном $n$ u $2 \operatorname{Re} \lambda \in \mathbb{Z}$ при нечетном $n$.

Разумеется, интересным является лишш случай $n<2$ max $\mathbf{t}$. Доказательство достигается при помощи того же приема [84], который привел к предложению 2.22 (1). Возможны обобшения на задачи с условиями сопряжения (трансмиссии) на гиперплоскостях, содержащих луч $\mathbb{L}_{+}$(ср. с [86]). Доступна и некоторая информация о собственных и присоединенных векторах, а для скалярных операторов в [79] найдены явные формулы.

Отметим, наконец, что разрешимость задач Соболева для обших систем и асимптотические свойства их решений изучались в [61], [83], [31; $§ 12.7]$.

\section{§. Краевые задачи в областях \\ с цилиндрическими выходами на бесконечность}

1. Краевые задачи. Рассмотрим задачу

$$
\begin{aligned}
& \mathscr{L}\left(x, \nabla_{x}\right) u(x)=f(x), \quad x \in \Omega, \\
& \mathscr{B}\left(x, \nabla_{x}\right) u(x)=g(x), \quad x \in \partial \Omega,
\end{aligned}
$$

в области $\Omega$, имеющей несколько цилиндрических выходов на бесконечность. Как обычно, для $u, v \in C_{0}^{\infty}(\bar{\Omega})^{T}$ справедлива формула Грина $(1.2)$ или (1.20) и пара $\{\mathscr{L}, a\}$ обладает полиномиальным свойством $1^{0}$, выдерживающим замораживание всюду в $\bar{\Omega}$. Коэффициенты дифференциальных операторов $\mathscr{L}\left(x, \nabla_{x}\right)$ и $\mathscr{B}\left(x, \nabla_{x}\right)$ гладкие. Уточним геометрические формы выходов. Введем цилиндры $\mathbb{Q}^{\mu}=$ $\left\{\left(y^{\mu}, z^{\mu}\right): y^{\mu} \in \omega^{\mu}, z^{\mu} \in \mathbb{R}\right\}$, где $\omega^{\mu} \subset \mathbb{R}^{n-1}$ - области с гладкими границами $\partial \omega^{\mu}$ и компактными замыканиями $\overline{\omega^{\mu}}$, а $x^{\mu}=\left(y^{\mu}, z^{\mu}\right)-$ системы декартовых координат (свои для каждого $\mu=1, \ldots, M)$. Предположим, что полуцилиндры $\mathbb{Q}_{+}^{\mu}=\left\{x^{\mu} \in \mathbb{Q}^{\mu}: z^{\mu}>0\right\}$ дизъюнктны, а область $\Omega$, имея гладкую $(n-1)$-мерную границу $\partial \Omega$, совпадает вне шара $\mathbb{B}_{R}^{n}=\{x:|x|<R\}$ с объединением $\mathbb{Q}_{+}^{1} \cup \cdots \cup \mathbb{Q}_{+}^{M}$, причем торцы $\omega^{\mu}(0)=\left\{x^{\mu} \in \mathbb{Q}^{\mu}: z^{\mu}=0\right\}$ погружены в $\Omega \cap \mathbb{B}_{R}^{n}$. 
Пусть $\mathscr{L}^{\mu}\left(x^{\mu}, \nabla_{x^{\mu}}\right)$ и $\mathscr{B}^{\mu}\left(x^{\mu}, \nabla_{x^{\mu}}\right)$ - дифференциальные операторы из (3.1) и (3.2), суженные на $\mathbb{Q}_{+}^{\mu} \backslash \mathbb{B}_{R}^{n}$ и $\partial \mathbb{Q}_{+}^{\mu} \backslash \mathbb{B}_{R}^{n}$ (система $x$ привязана к $\Omega$, и поэтому дополнительно требуется перезапись в координатах $\left.x^{\mu}\right)$. Будем считать, что при $z^{\mu} \rightarrow+\infty$ эти операторы с экспоненшиальной скоростью стабилизируются к операторам $L^{\mu}\left(y^{\mu}, \nabla_{y^{\mu}}, \partial_{z^{\mu}}\right)$ и $B^{\mu}\left(y^{\mu}, \nabla_{y^{\mu}}, \partial_{z^{\mu}}\right)$, т.е. для каждого из коэффишиентов $b\left(y^{\mu}, z^{\mu}\right)$ вьполняются соотношения

$$
\left|\nabla_{x^{\mu}}^{k}\left[b\left(y^{\mu}, z^{\mu}\right)-b^{\infty}\left(y^{\mu}\right)\right]\right| \leqslant c_{k} \exp \left(-\gamma_{\mu} z^{\mu}\right),
$$

где $\gamma_{\mu}>0$ и $k=0,1, \ldots$. Заменим $\partial_{z} \mu$ на $\lambda$ и получим аналогичный (2.10) полиномиальный пучок

$$
\mathbb{C} \ni \lambda \mapsto \mathfrak{A}^{\mu}(\lambda)=\left\{L^{\mu}\left(y^{\mu}, \nabla_{y^{\mu}}, \lambda\right), B^{\mu}\left(y^{\mu}, \nabla_{y^{\mu}}, \lambda\right)\right\}: \mathscr{D}^{l} H\left(\omega^{\mu}\right) \rightarrow \mathscr{R}^{l} H\left(\omega^{\mu}, \partial \omega^{\mu}\right),
$$

в котором векторные пространства $\mathscr{D}^{l} H(\cdot)$ и $\mathscr{R}^{l} H(\cdot)$ определены согласно $(1.26)$ и (1.27).

ЗАмечАниЕ 3.1. В согласии с [94], [95], [20], [31; § 3.4] все излагаемые результаты приспосабливаются к той ситуации, когда коэффициенты $b\left(y^{\mu}, z^{\mu}\right)$ стабилизируются к периодическим функциям $z^{\mu} \mapsto b^{\infty}\left(y^{\mu}, z^{\mu}\right)$. Разумеется, при этом множества $\mathbb{Q}^{\mu}$ могут иметь периодически изменяюшиеся сечения $\omega^{\mu}\left(z^{\mu}\right)$. Здесь мы пренебрегаем этим обобщением для упрошения формул, но еше вернемся к нему в п. $2 \S 5$.

2. Экспоненциальные решения. Пусть $\lambda$ - собственное число пучка $\mathfrak{A}^{\mu}$, а $\Phi^{0}, \ldots, \Phi^{s-1}$ - соответствуюшая жорданова цепочка длиной $s$, т.е. собственньй $\Phi^{0}$ и присоединенные $\Phi^{1}, \ldots, \Phi^{s-1}$ векторы удовлетворяют уравнениям

$$
\mathfrak{A}^{\mu}(\lambda) \Phi^{q}=-\sum_{h=1}^{q} \frac{1}{h !} \frac{d^{h} \mathfrak{A}^{\mu}}{d \lambda^{h}}(\lambda) \Phi^{q-h}, \quad q=0, \ldots, s-1,
$$

а уравнение с номером $q=s$ неразрешимо. Согласно [13], [83] указанные соотношения гарантируют, что функции

$$
\mathscr{U}^{\mu q}(y, z)=\exp (\lambda z) \sum_{h=0}^{q} \frac{1}{h !} z^{h} \Phi^{q-h}(y), \quad q=0, \ldots, s-1,
$$

являются решениями однородной модельной задачи $\left\{L^{\mu}, B^{\mu}\right\}$ в цилиндре $\mathbb{Q}^{\mu}$. Такие решения назьваем экспоненииальнылми даже в том случае, если $\lambda=0$ и множитель $\exp (\lambda z)$ в (3.5) исчезает. Пусть $P^{\mu}$ - линеал полиномов, соотнесенный с $\left\{L^{\mu}, a^{\mu}\right\}$ в $1^{0}$. Так как коэффициенты операторов $L^{\mu}, B^{\mu}$ и формы $a^{\mu}$ не зависят от $z^{\mu}$, линеал $P^{\mu}\left(B^{\mu}\right)$ характерен тем, что

$$
p \in P^{\mu}\left(B^{\mu}\right) \Rightarrow \partial p / \partial z^{\mu} \in P^{\mu}\left(B^{\mu}\right) .
$$

Отсюда вытекает, что в $P^{\mu}\left(B^{\mu}\right)$ имеется базис

$$
\left\{p^{\mu i k}: i=1, \ldots, I_{\mu}, k=0, \ldots, \tau_{\mu i}-1\right\},
$$


подчиненньй требованиям

$$
\begin{aligned}
& p^{\mu i 0} \text { не зависит от } z^{\mu}, \\
& \partial p^{\mu i k} / \partial z^{\mu}=p^{\mu i k-1} \text { при } k=1, \ldots, \tau_{\mu i}-1, i=1, \ldots, I_{\mu} .
\end{aligned}
$$

Нетрудно обнаружить, что в соответствии с (3.5) $\partial \mathscr{U}^{\mu q} / \partial z^{\mu}=\mathscr{U}^{\mu q-1}$ при $\lambda=0$, а значит, по базису (3.7) можно построить собственные и присоединенные векторы пучка $\mathfrak{A}^{\mu}$, отвечаюшие $\lambda=0$. Очередное утверждение показывает, что, как и в $\S 2$, такая операция достаточна для определения спектра пучка (3.4) в узкой полосе $\Pi^{\mu}=$ $\left\{\lambda \in \mathbb{C}:|\operatorname{Re} \lambda|<\delta_{\mu}\right\}$.

ПРЕДЛОЖЕНИЕ $3.2([31 ; \S 5.4],[33])$. Существует $\delta_{\mu}>0$, при котором вернъ следуюшие факты.

1) Eсли $\operatorname{dim} P^{\mu}\left(B^{\mu}\right)=0$, то полоса $\Pi^{\mu}$ свободна от спектра пучка $\mathfrak{A}^{\mu}$.

2) Пусть подпространство $P^{\mu}\left(B^{\mu}\right)$ нетривиально и дополнительно форма $a^{\mu}$ подчинена условию $3^{0}$ или $4^{0}$. Тогда в полосе $\Pi^{\mu}$ располохсено единственное собственное число $\lambda=0$, частные алгебраические кратности которого равнь $2 \tau_{\mu 1}, \ldots, 2 \tau_{\mu I_{\mu}}$. Базис (3.7) можсно виббрать так, чтобы жсордановы цепочки, составленные из следов полиномов $p^{\mu i 0}(\cdot), p^{\mu i s}(\cdot, 0), \ldots, p^{\mu i \tau_{\mu i}-1}(\cdot, 0)$, продолжсались до длин $2 \tau_{\mu i}$ и полученная таким образом система чепочек оказалась канонической.

3. Оператор задачи в экспоненциальных весовых классах. Через $W_{\beta}^{l}(\Omega)$ обозначим пополнение $C_{0}^{\infty}(\bar{\Omega})$ по норме

$$
\left\|w ; W_{\beta}^{l}(\Omega)\right\|=\left\|\mathbf{e}_{\beta} w ; H^{l}(\Omega)\right\|,
$$

где $\mathbf{e}_{\beta}$ - гладкая положительная функция в $\bar{\Omega}$, совпадаюшая на $\mathbb{Q}_{+}^{\mu} \backslash \mathbb{B}_{R}^{n}$ с $\exp \left(\beta z^{\mu}\right)$; $\mu=1, \ldots, M$. Как и в (2.4)-(2.6), определяются следовое $W_{\beta}^{l-1 / 2}(\partial \Omega)$ и векторные $\mathscr{D}_{\beta}^{l} W(\Omega), \mathscr{R}_{\beta}^{l} W(\Omega)$ весовые пространства. С задачей $(3.1),(3.2)$ связывается отображение

$$
\mathscr{A}_{\beta}^{l}=\{\mathscr{L}, \mathscr{B}\}: \mathscr{D}_{\beta}^{l} W(\Omega) \rightarrow \mathscr{R}_{\beta}^{l} W(\Omega)
$$

Если на прямой $i \mathbb{R}-\beta$ нет собственных чисел пучков $\mathfrak{A}^{1}, \ldots, \mathfrak{A}^{M}$, то оператор (3.9) фредгольмов (см. [96], [13], [20], [31; §5.1]). Так как только тривиальный полином квадратично суммируем в $\Omega$, благодаря полиномиальному свойству справедлива

Лемма 3.3. При $\beta \geqslant 0$ оператор $\mathscr{A}_{\beta}^{l}$ - мономорфизм, m.e. $\operatorname{ker} \mathscr{A}_{\beta}^{l}=\{0\}$.

Аналоги утверж дений 2.4 и 2.5 представлены, например, в [20], [31; 55.1$]$. Остается сформулировать результат.

ТЕОРема 3.4 ([31; 5.4$]$, [33]). 1) В условиях предложения $3.2(1), \mu=1, \ldots, M$, оператор $\mathscr{A}_{\beta}^{l}$ задачи (3.1), (3.2) является изоморфизмом при $|\beta|<\delta=$ $\min \left\{\delta_{1}, \ldots, \delta_{M}\right\} u l \geqslant \max \mathbf{t}$.

2) Пусть в случае $\operatorname{dim} P^{\mu}\left(B^{\mu}\right)>0$ выполнень условия предложсения 3.2 (2) $u \pm \beta^{ \pm} \in(0, \delta)$. Тогда операторы $\mathscr{A}_{\beta^{+}}^{l} u \mathscr{A}_{\beta^{-}}^{l}$ суть фредгольмовъ мономорфизм 
и эпиморфизм соответственно, т.е. $\operatorname{dim} \operatorname{ker} \mathscr{A}_{\beta^{+}}^{l}=\operatorname{dim}$ coker $\mathscr{A}_{\beta^{-}}^{l}=0$. Подпространства coker $\mathscr{A}_{\beta^{+}}^{l} u \operatorname{ker} \mathscr{A}_{\beta^{-}}^{l}$ имеют одинаковъе размерности $\tau$, где

$$
\tau=\tau_{1}+\cdots+\tau_{M}, \quad \tau_{\mu}=\tau_{\mu 1}+\cdots+\tau_{\mu I_{\mu}}
$$

4. Пространства с отделенной асимптотикой. В условиях теоремы 3.4 (2)

$$
\operatorname{dim} \operatorname{ker} \mathscr{A}_{\beta^{+}}^{l}=0, \quad \operatorname{dim} \text { coker } \mathscr{A}_{\beta^{+}}^{l}=\tau>0,
$$

и поэтому для построения фредгольмова оператора с нулевым индексом достаточно присоединить к $\mathscr{D}_{\beta^{+}}^{l} W(\Omega)$ подходящее подпространство размерностью $\tau$. Согласно предложению $3.2(2)$ эту роль могут взять на себя полиномиальные линеалы $P^{1}\left(B^{1}\right), \ldots, P^{M}\left(B^{M}\right)$, заведомо обладаюшие тем преимуществом, что они приводят к решениям $u$ с конечной "энергией” $a(u, u ; \Omega)$. Реализуем намеченное.

Пусть $\chi_{\mu} \in C^{\infty}(\bar{\Omega})$ - срезаюшая функция, равная единище на $\mathbb{Q}_{+}^{\mu} \backslash \mathbb{B}_{2 R}^{n}$ и имеюшая носитель в $\overline{\mathbb{Q}_{+}^{\mu}} \backslash \mathbb{B}_{R}^{n}$. Умножим каждый полином $p \in P^{\mu}\left(B^{\mu}\right)$ на $\chi_{\mu}$ и при $\beta>0$ образуем пространство

$$
\mathbf{D}_{\beta}^{l} W(\Omega)=\mathscr{D}_{\beta}^{l} W(\Omega) \dot{+} \chi_{1} P^{1}\left(B^{1}\right) \dot{+} \cdots \dot{+} \chi_{M} P^{M}\left(B^{M}\right)
$$

из функций, представимых в виде

$$
u=\tilde{u}+\sum_{*} c_{\mu i q} \chi_{\mu} p^{\mu i q},
$$

где $p^{\mu i q}$ - элементы базисов $(3.7), c_{\mu i q} \in \mathbb{C}$ и $\sum_{*}$ означает суммирование по $\mu=1, \ldots, M, i=1, \ldots, I_{\mu}$ и $q=0, \ldots, \tau_{\mu i}-1$. Кроме того,

$$
\left\|u ; \mathbf{D}_{\beta}^{l} W(\Omega)\right\|=\left(\left\|\tilde{u} ; \mathscr{D}_{\beta}^{l} W(\Omega)\right\|^{2}+\sum_{*}\left|c_{\mu i q}\right|^{2}\right)^{1 / 2},
$$

а изменение базисов или срезающих функций, разумеется, не влияет на $\mathbf{D}_{\beta}^{l} W(\Omega)$ ни алгебраически, ни топологически.

Пусть $0<\beta<\min \left\{\delta_{\mu}, \gamma_{\mu} \mid \mu=1, \ldots, M\right\}$, где $\gamma_{\mu}$ и $\delta_{\mu}$ - положительные числа, взятые из условий стабилизации (3.3) и из предложения 3.2. Так как полиномы из $P^{\mu}\left(B^{\mu}\right)$ аннулируются модельным оператором $\left\{\mathscr{L}^{\mu}, \mathscr{B}^{\mu}\right\}$, нетрудно убедиться в том, что оператор задачи (3.1), (3.2),

$$
\mathbf{A}_{\beta}^{l}: \mathbf{D}_{\beta}^{l} W(\Omega) \rightarrow \mathscr{R}_{\beta}^{l} W(\Omega),
$$

является непрерьвным при $l \leqslant \max \mathbf{t}$. Теперь вычисление Ind $\mathbf{A}_{\beta}^{l}$ и использование полиномиального свойства $1^{0}$ дает нужный результат. 
Tеорема 3.5 ([33]). Пусть выполнены условия теоремы 3.4 (2). Тогда при указанных ограничениях на $l$ и $\beta$ (фредгольмов) оператор (3.11) имеет нулевой индекс, причем ker $\mathbf{A}_{\beta}^{l}=\mathscr{P}(\mathscr{B})$ u coker $\mathbf{A}_{\beta}^{l}=\left\{\left(p, \mathscr{T} p, \mathscr{N}^{\prime} p\right): p \in \mathscr{P}(\mathscr{B})\right\}$, где $\mathscr{P}(\mathscr{B})=\{p \in \mathscr{P}: \mathscr{B} p=0$ на $\partial \Omega\}, \mathscr{P}$ - линеал полиномов из $1^{0}$, а для операторов простой структуры в формуле для коядра фигурирует пара $(p, \mathscr{T} p)$, отвечающая формуле Грина (1.7), а не тройка $\left(p, \mathscr{T} p, \mathscr{N}^{\prime \prime} p\right)$, возникшая согласно (1.19).

Во многих случаях особый интерес приобретает перечисление условий ортогональности, обеспечиваюших сушествование решения задачи (3.1), (3.2), экспоненциально затухающего на бесконечности. Благодаря общим результатам [15] для этого достаточно описать подпространство ker $\mathscr{A}_{\beta^{-}}^{l}$, уже встречавшееся в теореме 3.4 (2). Полиномиальное свойство дает полньй (но тривиальньй!) ответ лишь в ситуации теоремы 3.4 (1). Если же размерность ядра $\operatorname{ker} \mathscr{A}_{\beta^{-}}^{l}$ положительна, то множество $\mathscr{P}(\mathscr{B})$ не обязано исчерпьвать это ядро, т.е. могут появляться решения однородной задачи $(3.1),(3.2)$, растушие на бесконечности не быстрее $|x|^{k}$, но не являющиеся полиномами из $\mathscr{P}(\mathscr{B})$ (отметим, что в силу леммы 5.4 .9 [31] интеграл $a(u, u ; \Omega)$ из $(1.3)$ для таких решений расходится). Процедура их построения проста. Именно, линейная комбинация экспоненциальных решений (3.5) с $\lambda=0$, сформированная при учете предложения $3.2(2)$,

$$
\mathscr{W}(x)=\sum_{*} c_{\mu i q+\tau_{\mu i}} \chi_{\mu}(x) \mathscr{U}^{\mu i q+\tau_{\mu i}}(x)
$$

(cp. с (3.10)), подставляется в однородную задачу (3.1), (3.2) и отыскивается решение $w \in \mathbf{D}_{\beta}^{l} W(\Omega)$, компенсируюшее образовавшуюся невязку $\{\mathscr{F}, \mathscr{G}\} \in \mathscr{R}_{\beta}^{l} W(\Omega)$ (последнее включение верно потому, что экспоненциальные решения (3.5) аннулируются модельным оператором $\left\{\mathscr{L}^{\mu}, \mathscr{B}^{\mu}\right\}$ в $\left.\mathbb{Q}\right)$. Согласно теореме 3.5 количество условий разрешимости задачи для $w$ равно $\operatorname{dim} \mathscr{P}(\mathscr{B})$ и потому в $(3.12)$ остаются $\tau-\operatorname{dim} \mathscr{P}(\mathscr{B})$ свободных констант $c$, , перебор которых и доставляет базис в ортогональном дополнении $\mathscr{P}(\mathscr{B})$ до ядра ker $\mathscr{A}_{\beta^{-}}^{l}$. В несколько иной ситуации мы вернемся к этому вопросу в п. $2 \oint 5$.

В заключение отметим, что предложение 1.5 устанавливает равенство ker $\mathscr{A}_{\beta^{-}}^{l}=$ $\mathscr{P}(\mathscr{B})$ в том случае, когда оператор $\mathscr{L}$ с постоянными коэффициентами имеет простую структуру, на гранище $\partial \Omega$ назначены краевые условия Неймана $\mathscr{B}=\mathscr{N}$, а у области $\Omega$ есть лишь один выход на бесконечность.

5. Конкретные задачи. Рассмотрим ряд задач математической физики из п. $6 \S 1$, иллюстрируюших обшие теоремы этого параграффа.

ПримеР 3.6. Для трехмерной системы теории упругости базис (3.7), (3.8) в линеале жестких смещений (1.37) можно взять таким:

$$
\begin{gathered}
p^{h, 0}=e^{h}, \quad p^{h, 1}(y, z)=z e^{h}-y_{h} e^{3} \quad(h=1,2), \\
p^{3,0}=e^{3}, \quad p^{4,0}(y)=y_{2} e^{1}-y_{1} e^{2} .
\end{gathered}
$$

Следовательно, $I=4$ и $\tau_{1}=\tau_{2}=2, \tau_{3}=\tau_{4}=1$. Экспоненциальные решения (3.5), в которых $\lambda=0$ и $q=\tau_{i}, \ldots, 2 \tau_{i}-1, i=1, \ldots, 4$, имеют смысл полей смешений, порождающих в сечениях цилиндра $\mathbb{Q}=\omega \times \mathbb{R}^{1} \subset \mathbb{R}^{3}$ перерезьвающие и продольную 
силы и изгибаюшие и крутяший моменты (см. [97], [98] для изотропии и [31; $§ 5.7]$ в обшем случае). Условия сушествования решения $u \in W_{\beta^{+}}^{l+1}(\Omega)^{3}, \beta^{+} \in(0, \delta)$, задачи Неймана в полуограниченной цилиндрической области $\Omega$,

$$
(f, p)_{\Omega}+(g, p)_{\partial \Omega}=0 \quad \forall p \in \mathscr{P}=\mathscr{P}(\mathscr{N}),
$$

интерпретируются как самоуравновешенность нагружения $f, g$. В случае условий Дирихле (зажатая поверхность) $\operatorname{dim} \mathscr{P}(\mathscr{D})=0$ и справедлива теорема 3.4 (1). Вместе с тем, существуют и другие краевые условия, приводящие к однозначно разрешимой в $W_{\beta}^{l+1}(\Omega)^{3}$ задаче при $\beta \in(-\delta, \delta)$. Пусть, к примеру, область $\Omega$ с одним или несколькими выходами на бесконечность не является областью вращения, а на поверхности $\partial \Omega$ назначены линеаризованные условия Синьорини (контакт без трения)

$$
\nu \cdot u=0, \quad \nu \times \sigma^{(\nu)}(u)=0 \text { на } \partial \Omega
$$

(см. формулы $(2.42),(1.52)$ и пример 1.12$)$. Ни один из полиномов (3.13) не удовлетворяет условиям $(3.15)$ на $\partial \mathbb{Q}$, т.е. подпространства $P^{\mu}\left(B^{\mu}\right)$ и $\mathscr{P}(\mathscr{B})$ тривиальны, а значит, выполняются условия предложения 3.2 (1) и теоремы 3.4 (1). Если же $\Omega$ область вращения (т.е. $M=1$ или $M=2$, причем $\mathbb{Q}^{\mu}=\left\{x: r_{\mu}<|y|<R_{\mu}\right\}$ ), то упомянутые подпространства одномерные и содержат полином $p^{4,0}(y)=\left(y_{2},-y_{1}, 0\right)^{t}$, поворот вокруг оси цилиндров.

В [33] показано, как результаты, полученные в $\S 2$ и $\S 3$, переносятся на случай несамосопряженных оператор-матриц (1.34). В связи с этим обратимся к примеру 1.13.

ПримеР 3.7. Рассмотрим систему уравнений теории электроупругости, снабженную краевыми условиями $(2.58),(2.59)$. Так как электрический потенциал $\varphi=u_{4}$ удовлетворяет условиям Дирихле (2.59), возникает прежний линеал полиномов с базисом (3.13) (разумеется, $p^{h q}$ дополняются нулевой четвертой компонентой). Следовательно, теоремы 3.4 (2) и 3.5 приводят к тем же заключениям, что и в обычной теории упругости. Если заменить (2.59) условиями Неймана $\partial_{n} \varphi(x)=0, x \in \partial \Omega$, то за счет присоединения к базису (3.13) еше одного элемента $p^{5,0}=(0,0,0,1)^{t}$ размерность каждого из встречающихся линеалов прирастает на единицу.

Как упоминалось в п. $1 \S 2$, утверждения, опираюшиеся на полное полиномиальное свойство $3^{0}$, могут быть приспособлены к некоторым операторам сложной структуры (1.14). Продолжим обсуждение системы Стокса, имея в виду теорему 3.5.

Пример 3.8. 1) Для уравнений Стокса (1.45), (1.46) в цилиндре $\mathbb{Q}=\omega \times \mathbb{R} \subset \mathbb{R}^{3}$, снабженных условиями прилипания $u=0$ на $\partial \mathbb{Q}$, получаем $\mathscr{P}(\mathscr{D})=P(\mathscr{D})=\{0\}^{3} \times \mathbb{R}$, т.е. $\mathscr{U}^{0}(y, z)=(0,0,0,1)^{t}-$ постоянное давление. Предложение $3.2(2)$ при учете сказанного в примере 2.8 устанавливает, что $\varkappa=2 M$. Еще одно экспоненциальное решение (3.5) с $\lambda=0, q=1$ имеет вид

$$
\mathscr{U}^{1}(y, z)=(0,0,-\Psi(y), z)^{t}
$$

и описывает течение Пуазеля. В (3.16) $\Psi$ - решение задачи Дирихле для уравнения Пуассона на сечении $\omega$,

$$
-\Delta_{y} \Psi(y)=1, \quad y \in \omega ; \quad \Psi(y)=0, \quad y \in \partial \omega .
$$


2) Рассмотрим теперь в цилиндре $\mathbb{Q}$ краевую задачу $(1.45),(1.46),(1.52)$, считая, что сечение $\omega$ не является кругом или кольцом. В этом случае условие $\nu \cdot u=0$ на $\partial \mathbb{Q}$ исключает из $\mathscr{P}=\mathbb{R}^{4}$ постоянные поперечные составляюшие, т.е. $\mathscr{P}(\mathscr{B})=$ $P(\mathscr{B})=\left\{\left(0,0, c_{3}, c_{4}\right)^{t}: c_{q} \in \mathbb{R}\right\}$. Прямые вычисления показывают, что такие полиномы, постоянные давление и продольное течение, исчерпывают все экспоненциальные решения (3.5) при $\lambda=0$, т.е. собственное число $\lambda=0$ соответствующего пучка $\mathfrak{A}(\lambda)$ простое алгебраически и $\operatorname{ker} \mathfrak{A}(0)=P(\mathscr{B})$. Этот факт противоречит утверждению $3.2(2)$ и объясняется отсутствием аналога полного полиномиального свойства $4^{0}$ при постановке условий свободной поверхности (1.52). В самом деле, операторы краевых условий $\mathscr{D} v=v$ и $\mathscr{N} \mathbf{u}=\tau^{(\nu)}(\mathbf{u})$, проистекаюшие из формул Грина (1.48)-(1.50), не аннулируют полиномы $\mathbf{v}=(0,0,1,0)^{t}$ и $\mathbf{u}=(0,0,0,1)^{t}$ из $P(\mathscr{B})$, т.е. требование $4^{0}$ нельзя соблюсти даже при замене $\mathscr{N}$ на $\mathscr{D}$.

Если $\omega$ - область врашения, то к названньм экспоненциальным решениям добавляется пара полиномов

$$
\mathscr{U}^{30}(y)=\left(y_{2},-y_{1}, 0,0\right)^{t}, \quad \mathscr{U}^{31}(y, z)=\left(z y_{2},-z y_{1}, 0,0\right)^{t}
$$

порожденных жордановой цепочкой пучка $\mathfrak{A}$, имеющей длину 2 (ср. со сказанным в конце примера 3.6).

\section{§. Краевые задачи в тонких областях}

1. Исходная и предельная задачи. Пусть $\omega \subset \mathbb{R}^{n-d}$ и $S \subset \mathbb{R}^{d}$ - области с компактными замыканиями и гладкими границами. Введем множества, зависящие от малого параметра $\varepsilon>0$,

$$
\omega_{\varepsilon}=\left\{y \in \mathbb{R}^{n-d}: \eta=\varepsilon^{-1} y \in \omega\right\}, \quad \Omega(\varepsilon)=\omega_{\varepsilon} \times S .
$$

Семейство областей $\{\Omega(\varepsilon)\}_{\varepsilon \in(0,1)}$ называем тонкой областью и рассматриваем краевые задачи

$$
\begin{aligned}
& \mathscr{L}\left(\varepsilon, x, \nabla_{x}\right) u(\varepsilon, x)=f(\varepsilon, x), \quad x \in \Omega(\varepsilon), \\
& \mathscr{B}\left(\varepsilon, x, \nabla_{x}\right) u(\varepsilon, x)=g(\varepsilon, x), \quad x \in \Sigma(\varepsilon)=\partial \omega_{\varepsilon} \times S, \\
& \mathscr{D}\left(\varepsilon, x, \nabla_{x}\right) u(\varepsilon, x)=0, \quad x \in \Gamma(\varepsilon)=\omega_{\varepsilon} \times \partial S .
\end{aligned}
$$

Здесь $\mathscr{L}$ - оператор-матрица простой структуры, краевые условия (4.3) построены в соответствии с правилом (1.6), а $\mathscr{D}$ - система Дирихле из формулы Грина (1.2). Для оператора (1.10) краевые условия (4.4) эквивалентны таким:

$$
\nabla_{x}^{s} u_{k}(\varepsilon, x)=0, \quad x \in \Gamma(\varepsilon), \quad s=0, \ldots, t_{k}-1, \quad k=1, \ldots, T
$$

Для упрощения изложения считаем, что операторы $\mathscr{L}_{j k}$, составляющие матрищу $\mathscr{L}$, и форма $a$ имеют постоянные коэффициенты и являются однородными (т.е. $|\alpha|=t_{j}+t_{k}$ и $|\sigma|=t_{j},|\gamma|=t_{k}$ в (1.1) и (1.3)). Форма $а$ обладает полиномиальным свойством в полном объеме $\left(\right.$ см. $3^{0}$ и $\left.4^{0}\right)$. Благодаря сделанным допушениям это свойство конечно же выдерживает замораживание коэффициентов. 
ПримеР 4.1. Наиболее часто встречаюшиеся тонкие тела - стержень $\left(\omega \subset \mathbb{R}^{2}\right.$, $\left.S \subset \mathbb{R}^{1}\right)$ и пластина $\left(\omega \subset \mathbb{R}^{1}, S \subset \mathbb{R}^{2}\right)$; в гидродинамике такие геометрические формы ассоциируются с капиллярными и пленочныцми течениями. Сообразуясь с первой интерпретацией тонкой области $\Omega(\varepsilon)$, называем $\Sigma(\varepsilon)$ и $\Gamma(\varepsilon)$ боковой поверхностью и торцом соответственно. Кроме того, $\omega_{\varepsilon}$ и $S$ - сечения, поперечное и продольное.

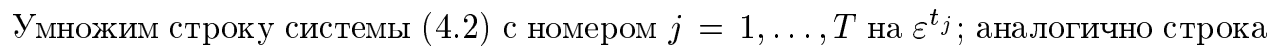
из (4.3) с номером $q=1, \ldots, m$ умножается на $\varepsilon^{r_{q}}$. Перейдем к "быстрым" переменным $\eta=\varepsilon^{-1} y$ (см. (4.1)) и заменим $u=\left(u_{1}, \ldots, u_{T}\right)$ на $U=\left(U_{1}, \ldots, U_{T}\right)$, где

$$
U_{k}(\eta, z)=\varepsilon^{-t_{k}} u_{k}(y, z), \quad k=1, \ldots, T .
$$

В результате таких преобразований операторы $\mathscr{L}$ и $\mathscr{B}$ расшепляются в суммы (конечные!) по степеням малого параметра $\varepsilon$

$$
\begin{aligned}
\varepsilon^{\mathbf{t}} \mathscr{L}\left(\nabla_{x}\right) \varepsilon^{\mathbf{t}} & =\sum_{h=0}^{\infty} \varepsilon^{h} L^{h}\left(\nabla_{\eta}, \nabla_{z}\right), \\
\varepsilon^{\mathbf{r}} \mathscr{B}\left(\varepsilon, x, \nabla_{x}\right) \varepsilon^{\mathbf{t}} & =\sum_{h=0}^{\infty} \varepsilon^{h} B^{h}\left(\eta, \nabla_{\eta}, \nabla_{z}\right) .
\end{aligned}
$$

Порядки операторов $L^{h}$ и $B^{h}$ относительно дифференцирования $\nabla_{z}$ не превосходят $h$. В частности,

$$
L^{0}\left(\nabla_{\eta}\right)=\mathscr{L}\left(\nabla_{\eta}, 0\right)
$$

а операторы $B^{0}\left(\eta, \nabla_{\eta}\right)\left(\right.$ и $\left.T^{0}\left(\eta, \nabla_{\eta}\right)\right)$ находятся согласно прежнему правилу (1.6) по операторам $N^{0}\left(\eta, \nabla_{\eta}\right)$ и $D^{0}\left(\eta, \nabla_{\eta}\right)$, фигурируюшим в формуле Грина вида (1.2), причем соответствуюшая форма $a^{0}$ получается из $a$ при помоши подстановок $\nabla_{y} \mapsto \nabla_{\eta}$ и $\nabla_{z} \mapsto(0, \ldots, 0) \in \mathbb{R}^{d}$.

Сделаем указанные замены в (4.2) и (4.3), а затем перейдем формально к $\varepsilon=0$. В итоге возникнет предельная краевая задача на поперечном сечении $\omega$, а зависимость от переменной $z \in S$ станет параметрической,

$$
L^{0}\left(\nabla_{\eta}\right) U(\eta, z)=F(\eta, z), \eta \in \omega ; \quad B^{0}\left(\eta, \nabla_{\eta}\right) U(\eta, z)=G(\eta, z), \eta \in \partial \omega .
$$

Форма $a^{0}$ наследует полное полиномиальное свойство от $a$, причем аналогично (2.30) соответствуюший линеал полиномов приобретает вид

$$
P^{0}=\{p(\eta): p \in \mathscr{P}, p(y, z) \text { не зависит от } z \in S\} .
$$

Итак, можно воспользоваться теоремой 1.9. 
Лемма 4.2. Задача (4.9) с правой частью $\{F, G\} \in \mathscr{R}^{l} H(\omega)$ имеет решение $U \in \mathscr{D}^{l} H(\omega)$ в том и только том случае, если

$$
(F, p)_{\omega}+\left(G, T^{0} p\right)_{\partial \omega}=0 \quad \forall p \in P^{0}\left(B^{0}\right) .
$$

Решение определено с точностью до слагаемого из линеала полиномов $P^{0}\left(B^{0}\right)=$ $\left\{p \in P^{0}: B^{0} p=0\right.$ на $\left.\partial \omega\right\}$.

В лемме 4.2 речь шла об операторе $\mathfrak{A}_{0}^{l}: \mathscr{D}^{l} H(\omega) \rightarrow \mathscr{R}^{l} H(\omega)$ задачи (4.9). Далее индекс $l$ не нужен и поэтому сократим обозначения:

$$
\begin{aligned}
& \mathfrak{A}_{0}=\left\{L^{0}\left(\nabla_{\eta}\right), B^{0}\left(\eta, \nabla_{\eta}\right)\right\} \\
& \mathfrak{A}_{q}\left(\nabla_{z}\right)=\left\{L^{q}\left(\nabla_{\eta}, \nabla_{z}\right), B^{q}\left(\eta, \nabla_{\eta}, \nabla_{z}\right)\right\}, \quad q=1,2, \ldots
\end{aligned}
$$

2. Квазицепочки. Пусть $\Phi^{1,0}, \ldots, \Phi^{J, 0}$ - ортонормированньй в $L_{2}(\omega)^{T}$ базис в ядре $\operatorname{ker} \mathfrak{A}_{0}$ упомянутого оператора $\mathfrak{A}_{0}$. По лемме 4.2 пары $\left\{\Phi^{1,0}, T^{0} \Phi^{1,0}\right\}, \ldots$, $\left\{\Phi^{J, 0}, T^{0} \Phi^{J, 0}\right\}$ образуют базис в подпространстве coker $\mathfrak{A}_{0}$. Введем проектор $\pi$ на образ $\operatorname{Im} \mathfrak{A}_{0}$,

$$
\begin{aligned}
\mathscr{R}^{l} H(\omega) \ni\{f, g\} & \mapsto \pi\{f, g\} \\
= & \left\{f-\sum_{j=1}^{T} \Phi^{j, 0}\left[\left(f, \Phi^{j, 0}\right)_{\omega}+\left(g, T^{0} \Phi^{j, 0}\right) \partial \omega\right], g\right\} \in \mathscr{R}^{l} H(\omega)
\end{aligned}
$$

Так как $\left(\Phi^{j, 0}, \Phi^{k, 0}\right)_{\omega}=\delta_{j, k}$, имеем $\pi^{2}=\pi$.

Заменим в (4.12) градиент $\nabla_{z}$ точкой $\theta \in \mathbb{S}^{d-1}$. Следуя [30], дополним каждьй элемент базиса до квазицепочки

$$
\left\{\Phi^{j, 0}, \Phi^{j, 1}(\theta), \Phi^{j, 2}(\theta), \ldots\right\}, \quad j=1, \ldots, J
$$

соблюдая при этом следующие правила: во-первых, $\theta \mapsto \Phi^{j, q}(\theta)$ - однородный полином степени $q$, во-вторых, выполнены условия ортогональности

$$
\left(\Phi^{j, q}(\theta), \Phi^{k, 0}\right)_{\omega}=0, \quad j, k=1, \ldots, J, \quad q=1,2, \ldots
$$

и, в-третьих, верны равенства

$$
\mathfrak{A}_{0} \Phi^{j, q}(\theta)=-\pi \sum_{i=1}^{q} \mathfrak{A}_{i}(\theta) \Phi^{j, q-i}(\theta), \quad q=0,1, \ldots
$$

Подчеркнем, что для краткости у $\Phi^{j, q}$ и $\mathfrak{A}_{q}$ опускаются аргументы $\eta$ и $\nabla_{\eta}$. В силу (4.13) правая часть формулы (4.16) удовлетворяет условиям разрешимости (4.11), т.е. лемма 4.2 гарантирует сушествование квазищепочек (4.14), подчиненшых указанным требованиям. Подсчет степеней полиномов $\theta \mapsto \Phi^{j, q}(\theta)$ производится по индукции при учете сказанного о коэффициентах расщеплений (4.7). 
Бесконечные квазицепочки (4.14) построены так, что вьполняется формальное (асимптотическое) равенство

$$
\sum_{h=0}^{\infty} \varepsilon^{h} \mathfrak{A}_{h}(\theta) \sum_{p=0}^{\infty} \varepsilon^{p} \Phi^{j, p}(\theta)=\sum_{k=1}^{T} \sum_{q=1}^{\infty} \varepsilon^{q} \mathfrak{M}_{k j}^{q}(\theta)\left\{\Phi^{k, 0}, 0\right\},
$$

в котором

$$
\begin{gathered}
\mathfrak{M}_{k j}^{q}(\theta)=\left(\mathfrak{F}^{j q}(\theta), \Phi^{k, 0}\right)_{\omega}+\left(\mathfrak{G}^{j q}(\theta), T^{0} \Phi^{k, 0}\right)_{\partial \omega} \\
\left\{\mathfrak{F}^{j q}(\theta), \mathfrak{G}^{j q}(\theta)\right\}=\sum_{i=1}^{q} \mathfrak{A}_{i}(\theta) \Phi^{j, q-i}(\theta) .
\end{gathered}
$$

Члены суммы из правой части (4.17) лежат вне $\operatorname{Im} \mathfrak{A}_{0}$ и поэтому не могут быть компенсированы при помоши решений предельной задачи (4.9) на поперечном сечении $\omega$. Центральная идея построения асимптотики решения задачи (4.2)-(4.4) в тонкой области заключается в формировании из операторов $\mathfrak{M}_{k j}^{q}\left(\nabla_{z}\right)$ результирующей задачи на продольном сечении $S$, за счет которой можно было бы добиться нужных ортогональностей и решить возникаюшие предельные задачи на $\omega$ полностью, без проецирования на $\operatorname{Im} \mathfrak{A}_{0} \ldots$. . В [44], [45], [30] исследование проводилось в значительно более общей ситуации (переменные коэффициенты, быстроосциллирующая граница и пр.). Сужение постановки задачи при планомерном использовании полиномиального свойства позволяет дать исчерпываюшее описание асимптотических структур.

Цепочки (4.14) имеют правильное строение, если можно найти набор $\left\{\tau_{1}, \ldots, \tau_{J}\right\}$ их фиктивных полудлин, вьполнив при этом два условия: во-первых,

$$
\mathfrak{M}_{k j}^{q}(\theta)=0 \quad \text { при } q<\tau_{k}+\tau_{j} ; \quad j, k=1, \ldots, J, \quad \theta \in \mathbb{S}^{d-1}
$$

и, во-вторых, $(J \times J)$-матрица $\mathfrak{M}(\theta)=\left(\mathfrak{M}_{k j}^{\tau_{k}+\tau_{j}}(\theta)\right)$ оказьвается неособенной, т.е.

$$
\operatorname{det} \mathfrak{M}(\theta) \neq 0 \text { при } \quad \theta \in \mathbb{S}^{d-1} \text {. }
$$

Если эти требования соблюдены, основной член асимптотики решения $u(\varepsilon, x)$ задачи (4.2)-(4.4) ищем как сумму

$$
\mathscr{U}^{0}(\varepsilon, x)=\sum_{j=1}^{J} \varepsilon^{-\tau_{j}-\tau_{\max }} \sum_{q=0}^{\tau_{j}+\tau_{\max }} \varepsilon^{q+\mathbf{t}} \Phi^{j, q}\left(\eta, \nabla_{z}\right) v_{j}(z)+\varepsilon^{\mathbf{t}} U^{0}(\eta, z),
$$

где $\tau_{\max }=\max \left\{\tau_{1}, \ldots, \tau_{J}\right\}$, а новая неизвестная функция $v=\left(v_{1}, \ldots, v_{J}\right)^{t}$ предназначена для удовлетворения условий разрешимости (4.11) предельной задачи (4.9) для $U^{0}$. Многие члены невязок, возникающих при подстановке (4.21) в (4.2) и (4.3), исчезают благодаря формулам (4.19). Для устранения первых ненулевых слагаемых

$$
\varepsilon^{-\tau_{\max }+\tau_{k}} \mathfrak{M}_{k j}^{\tau_{k}+\tau_{j}}\left(\nabla_{z}\right) v_{j}(z)\left\{\Phi^{k, 0}(\eta), 0\right\}
$$


образуем результирующую систему

$$
\mathfrak{M}\left(\nabla_{z}\right) v(z)=\mathfrak{F}(z), \quad z \in S,
$$

оказываюшуюся эллиптической ввиду требования (4.20). В согласии с краевыми условиями (4.4) или (4.5) на торце $Г(\varepsilon)$ тонкой области $\Omega(\varepsilon)$ снабдим систему (4.23) условиями Дирихле

$$
\nabla_{z}^{k} v_{j}(z)=0, \quad z \in \partial S, \quad k=0, \ldots, \tau_{j}-1, \quad j=1, \ldots, J .
$$

Осталось выписать выражения для компонент $\mathfrak{F}_{1}, \ldots, \mathfrak{F}_{J}$ функции $\mathfrak{F}$. Предположим, что функции $f$ и $g$ в (4.2) и (4.3) допускают представления

$$
f(\varepsilon, x)=\varepsilon^{-\mathbf{t}} f^{0}(\eta, z)+\tilde{f}(\varepsilon, x), \quad g(\varepsilon, x)=\varepsilon^{-\mathbf{r}} g^{0}(\eta, z)+\tilde{g}(\varepsilon, x)
$$

с малыми остатками $\tilde{f}$ и $\tilde{g}$. В правую часть задачи $(4.9)$ для $U^{0}$ поместим проекцию $\left\{F^{0}, G^{0}\right\}=\pi\left\{f^{0}, g^{0}\right\}$ и положим

$$
\begin{gathered}
\mathfrak{F}_{j}(z)=0 \text { при } \tau_{j}<\tau_{\max }, \\
\mathfrak{F}_{j}(z)=\left(f^{0}(\cdot, z), \Phi^{j, 0}\right)_{\omega}+\left(g^{0}(\cdot, z), T^{0} \Phi^{j, 0}\right)_{\partial \omega} \text { при } \tau_{j}=\tau_{\max } .
\end{gathered}
$$

Фрагменты проекции $(\mathbb{I}-\pi)\left\{f^{0}, g^{0}\right\}$, не упомянутые в (4.26), и члены (4.18) рядов (4.17), следуюшие за (4.22), учитываются при формировании младших асимптотических слагаемых. Обоснование главного члена (4.21) асимптотики и сопутствуюшие вопросы (разрешимость задачи $(4.23),(4.24)$, оценки остатков в (4.25)) обсуждаются далее в п. 4.

3. Полиномиальное свойство и квазицепочки. Основные атрибуты результирующей системы (4.23) можно описать в терминах линеала полиномов

$$
\mathscr{P}(\mathscr{B})=\left\{p(\eta, z): p \in \mathscr{P}, \mathscr{B}\left(\varepsilon, x, \nabla_{x}\right) p(y, z)=0,(y, z) \in \Sigma(\varepsilon)\right\} .
$$

Зафиксируем точку $\theta \in \mathbb{S}^{d-1}$ и положим

$$
\begin{aligned}
& P^{\theta}(\mathscr{B})=\{p(\eta, z): \text { полином } p \in \mathscr{P}(\mathscr{B}) \text { зависит лишь } \\
& \text { от переменных } \left.\eta \in \omega \text { и } t=\theta_{1} z_{1}+\cdots+\theta_{d} z_{d} \in \mathbb{R}\right\} .
\end{aligned}
$$

Иными словами, “слой” $\omega \times \mathbb{R}^{d}$ представлен как объединение цилиндров $\mathbb{Q}^{\theta}=\omega \times \mathbb{R}$ с осями, проходящими через точки единичной сферы $\mathbb{S}^{d-1}$, и аналогично п. $2 \S 3$ сооружено подпространство полиномов (4.28), обслуживаюшее модельную задачу $\left\{\mathscr{L}\left(\nabla_{\eta}, \theta \partial_{t}\right), \mathscr{B}\left(\eta, \nabla_{\eta}, \theta \partial_{t}\right)\right\}$ в $\mathbb{Q}^{\theta}$. Ясно, что $P^{0}\left(B^{0}\right) \subset P^{\theta}(\mathscr{B})$. Кроме того, подобно формуле (3.6) дифференцирование $\partial_{t}=\partial / \partial t$ не выводит полином из подпространства $P^{\theta}(\mathscr{B})$ и поэтому в нем можно найти базис

$$
\left\{p_{\theta}^{i, k}: i=1, \ldots, J, k=0, \ldots, \tau_{i}(\theta)-1\right\}
$$

(cр. с (3.7) и (3.8)), характеризуюшийся тем, что

$$
p_{\theta}^{i, 0} \in P^{0}\left(B^{0}\right), \quad \partial_{t} p_{\theta}^{i, k}=p_{\theta}^{i, k}, \quad k=1, \ldots, \tau_{i}(\theta)-1 .
$$

Ключевьм является следуюшее предположение.

$6^{0}$. Базисы (4.29), (4.30) можсно выбрать так, чтобы числа $\tau_{i}=\tau_{i}(\theta)$ и полиномы $p^{i, 0}=p_{\theta}^{i, 0}$ не зависели от точки $\theta \in \mathbb{S}^{d-1}$. 
ПримеР 4.3. Рассмотрим систему уравнений теории упругости с краевыми условиями Неймана $\mathscr{B}=\mathscr{N}$ (см. пример 1.12), для которой $\mathscr{P}(\mathscr{N})$ - шестимерньй линеал жестких смещений (1.37).

1) В случае тонкого стержня $\Omega(\varepsilon)=\omega_{\varepsilon} \times(-1,1) \subset \mathbb{R}^{2} \times \mathbb{R}^{1}$ нужньй базис в $P^{\theta}(\mathscr{N})=\mathscr{P}(\mathscr{N})$ вьглядит так:

$$
\begin{aligned}
& p^{i, 0}=e^{i}, \quad p_{\theta}^{i, 1}(y, \theta z)=\theta\left(z e^{i}-y_{i} e^{3}\right), \quad i=1,2, \\
& p^{3,0}=e^{3}, \quad p^{4,0}(y)=y_{2} e^{1}-y_{1} e^{2} .
\end{aligned}
$$

Здесь $\theta= \pm 1-$ точка 0 -мерной сферы $\mathbb{S}^{0}$. Таким образом, $J=4$ и $\tau_{1}=\tau_{2}=2$, $\tau_{3}=\tau_{4}=1$.

2) Тонкую пластину обычно располагают горизонтально, что приводит к изменению ориентации осей по сравнению с использованной в (4.1). Именно, считается, что срединное сечение $\omega \subset \mathbb{R}^{2}$ пластины $\Omega(\varepsilon)=\omega \times S_{\varepsilon}$ лежит в плоскости с координатами $y=\left(y_{1}, y_{2}\right)$, а поперечное направление с ортом $e^{3}$ обозначается $z$ (нужно в (4.1) переставить пары $y, \omega$ и $z, S$, а быструю переменную ввести так: $\zeta=\varepsilon^{-1} z \in S=$ $(-1 / 2,1 / 2))$. Подпространство $P^{\theta}(\mathscr{N})$ является четырехмерным и в новых обозначениях искомый базис при $\theta=\left(\theta_{1}, \theta_{2}\right)=|y|^{-1} y \in \mathbb{S}^{1}$ приобретает вид

$$
\begin{array}{ll}
p^{1,0}=e^{1}, & p^{2,0}=e^{2}, \\
p^{3,0}=e^{3}, & p^{3,1}(y, z)=e^{3}\left(\theta_{1} y_{1}+\theta_{2} y_{2}\right)-z\left(e^{1} \theta_{1}+e^{2} \theta_{2}\right) .
\end{array}
$$

В итоге $J=3$ и $\tau_{1}=\tau_{2}=1, \tau_{3}=2$.

Обсудим требование $6^{0}$. Оно не является ограничительным при $d=1$, поскольку $\mathbb{S}^{d-1}=\{ \pm 1\}$ и два возникающих случая $t= \pm z$ по сушеству не различаются. Более того, $\Omega(\varepsilon)$ - тонкий цилиндр и легко обнаружить как полную тождественность формул $(4.29),(4.30)$ и $(3.7),(3.8)$, так и аналогии в конструкциях систем жордановых цепочек (п. $2 \S 3)$ и квазицепочек (4.17). Именно, если взять базис $\left\{p^{j, k}: j=1, \ldots, J, k=\right.$ $\left.0, \ldots, \tau_{j}-1\right\}$, назначенный предложением $3.2(2)$ для линеала $P^{(+1)}(\mathscr{B})$, то соответствуюшая каноническая система жордановых цепочек окажется ничем иным, как системой квазицепочек $(\theta=+1)$ с блочно диагональной матрицей $\mathfrak{M}(+1)$.

При $d>1$ ситуация осложняется тем, что элементы квазицепочек обязаны быть полиномами переменных $\theta_{1}, \ldots, \theta_{d}-$ в противном случае не удается образовать $\partial u \phi$ ференииальный оператор $\mathfrak{M}\left(\nabla_{z}\right)$. Предложение $3.2(2)$ доставляет систему жордановых цепочек в каждой из точек $\theta \in \mathbb{S}^{d-1}$, но, разумеется, с неконтролируемой зависимостью от $\theta$. Требование $5^{0}$ устраняет $\theta$ из основных характеристик системы (собственные векторы и частные нулевые кратности) и, тем самым, позволяет построить по индукции квазищепочки (4.14) с нужными свойствами.

Teорема 4.4 ([30]). Пусть в дополнение $\kappa 3^{0}$ и $4^{0}$ выполнено требование $6^{0}$. Тогда существует система квазищепочек $\left\{\Phi^{j, q}(\eta, \theta): j=1, \ldots, J, q=0,1 \ldots\right\}$ с правильной структурой, порожденной фиктивными полудлинами $\tau_{1}, \ldots, \tau_{J}$. Если $z \mapsto v_{j}(z)$ - полином степени не выше $\tau_{j}-1$, то сумма

$$
\Phi^{j, 0}(\eta) v_{j}(z)+\Phi^{j, 1}\left(\eta, \nabla_{z}\right) v_{j}(z)+\cdots+\Phi^{j, \tau_{j}-1}\left(\eta, \nabla_{z}\right) v_{j}(z)
$$




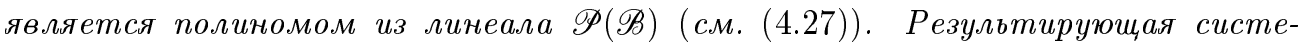
ма (4.23) оказывается эллиптической и формально самосопряэсенной, а ее размеры $J \times J$ и порядки элементов оrd $\mathfrak{M}_{k j}\left(\nabla_{z}\right)=\tau_{k}+\tau_{j}$ находятся по базису (4.29), (4.30) в линеале полиномов $P^{\theta}(\mathscr{B})$ из (4.28).

Все известные автору стационарные задачи математической физики в тонких областях характерны тем, что результирующая задача наследует полиномиальное свойство от исходной. Вопрос о справедливости такого утверждения для общих краевых задач (4.2)-(4.4) остается открытым.

ПримеР 4.5. В условиях, описанных перед примером 2.8, утверждения этого параграфа сохраняют силу и для оператор-матриц с усложненной структурой (1.14). Поэтому правомочно применить теорему 4.4 к задачам Стокса о тонких течениях, пленочных и капиллярных. Так как линеалы $\mathscr{P}(\mathscr{D})$ и $P^{\theta}(\mathscr{D})$ во всех случаях совпадают с $\{0\}^{3} \times \mathbb{R}$ (постоянное давление), требование $5^{0}$ очевидно и $J=1, \tau_{1}=1$. Результируюшее скалярное уравнение второго порядка назьвается уравнением Рейнольдса (см. [99], [100] для $n=3, d=2$ и [101] для $n=3, d=1)$.

В теории тонких пластин, упругих или электропроводящих, предельные задачи (4.9) упрощаются до обыкновенных дифференциальных уравнений с постоянными коэффициентами и потому допускают явные решения. Это обстоятельство позволило создать в [45] компьютерную программу, производящую редукиию размерности и вычисляющую результирующие задачи для произвольных систем уравнений второго порядка. Благодаря полиномиальному свойству и матричной записи (1.10) систем в настоящее время упомянутая программа существенно усовершенствована (совместно с О.В. Мотыгиным) и вся процедура сведена к простым алгебраическим операциям над матрицами. Приведем некоторые результаты вычислений.

ПримеР 4.6. 1) Рассмотрим систему уравнений пњезоупругости (пример 1.13) с краевыми условиями Неймана на основаниях $\Sigma^{ \pm}(\varepsilon)=\omega \times\{ \pm \varepsilon / 2\}$ пластины $\Omega(\varepsilon)=$ $\omega \times(-\varepsilon / 2, \varepsilon / 2) \subset \mathbb{R}^{3}$. Для того чтобы записать результирующую систему в компактной форме, переставим столбцы в матрице $\Lambda(\xi)$ из $(1.40)$ и положим

$$
\Lambda(\xi)^{t}=\left(\begin{array}{ccccccccc}
\xi_{1} & 0 & \alpha \xi_{2} & 0 & 0 & \alpha \xi_{3} & 0 & 0 & 0 \\
0 & \xi_{2} & \alpha \xi_{1} & 0 & 0 & 0 & \alpha \xi_{3} & 0 & 0 \\
0 & 0 & 0 & 0 & 0 & \alpha \xi_{1} & \alpha \xi_{2} & \xi_{3} & 0 \\
0 & 0 & 0 & \xi_{1} & \xi_{2} & 0 & 0 & 0 & \xi_{3}
\end{array}\right), \quad \alpha=\frac{1}{\sqrt{2}}
$$

Соответствуюшим образом видоизменяется и (числовая) матрица $Q$, которую теперь удобно разбить на блоки $Q_{11}, Q_{22}$ и $Q_{12}=Q_{21}^{t}$ размерами $5 \times 5,4 \times 4$ и $5 \times 4$. Введем еще матрищы

$$
\begin{aligned}
\mathfrak{L}(\xi) & =\left(\begin{array}{cccccccc}
\xi_{1} & 0 & \alpha \xi_{2} & 0 & 0 & 0 & 0 & 0 \\
0 & \xi_{2} & \alpha \xi_{1} & 0 & 0 & 0 & 0 & 0 \\
0 & 0 & 0 & 0 & 0 & \alpha \xi_{1}^{2} & \alpha \xi_{2}^{2} & \xi_{1} \xi_{2} \\
0 & 0 & 0 & \xi_{1} & \xi_{2} & 0 & 0 & 0
\end{array}\right) \\
\mathfrak{Y}(\zeta) & =\left(\begin{array}{cccccccc}
1 & 0 & 0 & 0 & 0 & -\alpha^{-1} \zeta & 0 & 0 \\
0 & 1 & 0 & 0 & 0 & 0 & -\alpha^{-1} \zeta & 0 \\
0 & 0 & 1 & 0 & 0 & 0 & 0 & -\alpha^{-1} \zeta \\
0 & 0 & 0 & 1 & 0 & 0 & 0 & 0 \\
0 & 0 & 0 & 0 & 1 & 0 & 0 & 0
\end{array}\right)
\end{aligned}
$$


Оператор $\mathfrak{M}\left(\nabla_{y}\right)$ результируюшей системы принимает вид $\mathfrak{L}\left(-\nabla_{y}\right)^{t} \mathfrak{Q} \mathfrak{L}\left(\nabla_{y}\right)$, a $(8 \times 8)$ матрища $\mathfrak{Q}$ находится по формуле

$$
\mathfrak{Q}=\int_{-1 / 2}^{1 / 2} \mathfrak{Y}(\zeta)^{t}\left(Q_{11}-Q_{12} Q_{22}^{-1} Q_{21}\right) \mathfrak{Y}(\zeta)^{t} d \zeta
$$

(разумеется, интегрирование по $\zeta$ вьполнить нетрудно). Матришы (4.34) и (4.35) алгебраически комплектны. По-прежнему выгоден переход к комплексной форме записи (1.34) - после введения мнимого электрического потенциала $i \varphi$ результируюшая задача наследует полиномиальное свойство от исходного оператора $\mathscr{Q}$, и заключения о ее разрешимости делаются на основе теоремы 1.9 и предложения 1.11 .

$2)$ Если на основаниях пластины $\Sigma^{ \pm}(\varepsilon)$ назначить краевые условия $(2.58),(2.59)$, то результируюшая система уменшшается до размеров $6 \times 6$. При этом у матрищ $(4.35)$ и (4.36) сохраняются лишь три верхних строки и исчезают четвертый и пятый столбцы (иными словами, $\mathfrak{L}(\xi)$ преврашается в матришу $\mathfrak{L}^{\prime}(\xi)$, определенную согласно (1.41)). Кроме того, блоки $Q_{11}, Q_{22}$ и $Q_{12}=Q_{21}^{t}$ урезаются до размеров $3 \times 3$ путем вычеркивания "лишних" нижних строк и правых столбцов. Наконец, после вычисления интеграла $(4.37)$ находим $(6 \times 6)$-матрицу $\mathfrak{Q}^{\prime}$ (усеченную матрицу $\left.\mathfrak{Q}\right)$, которая оказьвается блочно-диагональной

$$
\mathbf{Q}^{\prime}=\operatorname{diag}\left\{\mathbf{Q}, \frac{1}{6} \mathbf{Q}\right\}, \quad \mathbf{Q}=Q_{11}^{\prime}-Q_{12}^{\prime}\left(Q_{22}^{\prime}\right)^{-1} Q_{21}^{\prime} .
$$

Итак, условия Дирихле (2.59) устраняют электрический потенциал $\varphi$ из асимптотических конструкций и возникаюшая результируюшая система не отличается от упоминавшейся в примере 1.14. Матрицы $\mathfrak{L}^{\prime}(\xi)$ и $\mathfrak{Q}^{\prime}$ из оператора $\mathfrak{M}^{\prime}\left(\nabla_{y}\right)=\mathfrak{L}^{\prime}\left(-\nabla_{y}\right)^{t} \mathfrak{Q}^{\prime} \mathfrak{L}^{\prime}\left(\nabla_{y}\right)$, описывающего деф̆ормацию тонкой упругой пластины, определяются по формулам (1.41) и (4.38), где $(3 \times 3)$-матрицы $Q_{11}^{\prime}, Q_{22}^{\prime}$, $Q_{12}^{\prime}$ составляются из упругих модулей $A_{j k}$ (элементов $(6 \times 6)$-матрищы Гука, фигурировавшей в примере 1.12) следуюшим образом:

$$
\left(\begin{array}{lll}
A_{11} & A_{12} & A_{16} \\
A_{21} & A_{22} & A_{26} \\
A_{61} & A_{62} & A_{66}
\end{array}\right), \quad\left(\begin{array}{lll}
A_{55} & A_{54} & A_{53} \\
A_{45} & A_{44} & A_{43} \\
A_{35} & A_{34} & A_{33}
\end{array}\right), \quad\left(\begin{array}{lll}
A_{15} & A_{14} & A_{13} \\
A_{25} & A_{24} & A_{23} \\
A_{65} & A_{64} & A_{63}
\end{array}\right) .
$$

При различных ограничениях на симметрию упругих свойств подобные (4.38) формулы вьводились в рамках технической теории пластин, основанной на классических гипотезах Кирхгофа (см. [102], [103] и др.) или путем формального асимптотического анализа (см. [104], [105] и др.). Результаты, приведенные в следуюшем разделе, доставляют обоснование асимптотических представлений и тем самым подтверждают справедливость гипотез Кирхгофа. Неожиданньм является то, что ввиду блочной структуры матрипы $\mathfrak{Q}^{\prime}$ из (4.38) без каких-либо предположений о симметрии результируюшая задача распадается на две независимых, описывающих плоское напряженное состояние (вектор $\left.\left(w_{1}, w_{2}\right)\right)$ и изгиб пластины (скаляр $\left.w_{3}\right)$. В изотропном случае (см. (1.39)) первая задача содержит двумерную систему Ламе с постоянными $\mu$ и $\lambda^{\prime}=2 \lambda \mu(\lambda+2 \mu)^{-1}$, а вторая - бигармонический оператор $D \Delta^{2}$, где $D=\mu(\lambda+\mu)[6(\lambda+2 \mu)]^{-1}$ - приведенная цилиндрическая жесткость пластины. 
4. Весовое неравенство Корна и обоснование асимптотики. Пусть $\mathscr{L}$ оператор (1.10). Благодаря условиям Дирихле (4.4), соотношениям (1.12) и предложению 1.11 выполняется оценка

$$
a(u, u ; \Omega(\varepsilon)) \geqslant c(\varepsilon)\left\|u ; \mathscr{D}^{0} H(\Omega(\varepsilon))\right\|^{2} .
$$

Величина $c(\varepsilon)>0$ зависит от малого параметра $\varepsilon>0$, и для оправдания асимптотических конструкций в общей ситуации требуется уточнить и саму оценку (4.39), и структуру квазищепочек $\left\{\Phi^{j, q}\right\}$. Пусть сушествуют такие целые положительные числа $h_{1}, \ldots, h_{T}$, что для любой функции $u \in \mathscr{D}^{0} H(\Omega(\varepsilon))$, подчиненной условиям Дирихле (4.5), справедливо весовое неравенство Корна

$$
a(u, u ; \Omega(\varepsilon)) \geqslant c|u|^{2},
$$

в котором постоянная $c>0$ не зависит от $u$ и $\varepsilon \in(0,1)$,

$$
|u|=\left(\sum_{k=1}^{T} \sum_{|\sigma|+|\gamma| \leqslant t_{k}} \varepsilon^{2\left(h_{k}-t_{k}+|\sigma|\right)}\left\|d_{\varepsilon}^{|\gamma|-h_{k}} \nabla_{y}^{\sigma} \nabla_{z}^{\gamma} u_{k} ; L_{2}(\Omega(\varepsilon))\right\|^{2}\right)^{1 / 2}
$$

a $d_{\varepsilon}(z)=\varepsilon+\operatorname{dist}\{z, \partial S\}$-весовой множитель. Предположим еще, что после перенумерации компонент и цепочек (упорядочения чисел в наборах $\left\{h_{1}, \ldots, h_{T}\right\}$ и $\left\{\tau_{1}, \ldots, \tau_{J}\right\}$ ) оказалось, что

$$
\begin{aligned}
s_{\mu}=\tau_{1+J^{\mu-1}}=\cdots= & \tau_{J^{\mu}}=h_{1+T^{\mu-1}}=\cdots=h_{T^{\mu}}, \quad \mu=1, \ldots, m, \\
& s_{1}<s_{2}<\cdots<s_{m},
\end{aligned}
$$

(где $0=J^{0}<J^{1}<\cdots<J^{m}=J$ и $0=T^{0}<T^{1}<\cdots<T^{m}=T$ ) и, кроме того, справедливы равенства

$$
\Phi_{k}^{j, q}(\eta, \theta)=0 \text { при } h_{k}<\tau_{j}, q<\tau_{j}-h_{k} .
$$

Подчеркнем, что согласно (4.42) наборы $\left\{\tau_{1}, \ldots, \tau_{J}\right\},\left\{h_{1}, \ldots, h_{T}\right\}$ и $\left\{s_{1}, \ldots, s_{m}\right\}$ совпадают как множества.

Лемма 4.7 ([30]). При сделанных предположсениях (4.42), (4.41) результирующая задача (4.23), (4.24) имеет единственное решение $v \in H^{l+\tau_{1}}(S) \times \cdots \times$ $H^{l+\tau_{J}}(S)$ при любой правой части $\mathfrak{F} \in H^{l-\tau_{1}}(S) \times \cdots \times H^{l-\tau_{J}}(S), l \geqslant \tau_{\max }, u$ справедлива соответствующая оценка $\|v ; \cdot\| \leqslant c\|\mathfrak{F} ; \cdot\|$.

Теорема 4.8 ([30]). Пусть в дополнение $к$ сформулированным требованиям краевые условия (4.3) однородные, функиия $f^{0}$ из представления (4.25) гладкая на $\bar{\omega} \times \bar{S}$, а для остатка $\tilde{f}$ верно неравенство

$$
\sum_{j=1}^{T} \varepsilon^{t_{j}-h_{j}}\left\|d_{\varepsilon}^{h_{j}} \tilde{f}_{j} ; L_{2}(\Omega(\varepsilon))\right\| \leqslant c_{0} \varepsilon^{\delta_{0}+(n-d) / 2}
$$


где постоянная $c_{0}$ не зависит от $\varepsilon$, а $\delta_{0}$ - некоторое положстельное число. Тогда решение и исходной задачи (4.23), (4.24) в тонкой области $\Omega(\varepsilon)$ и асимптотическое приближение (4.21), построенное по решению $v$ результирующей задачи (4.23), (4.24), связань соотношением

$$
\|u-\mathscr{U}\| \leqslant c_{\delta} \varepsilon^{\delta+(n-d) / 2}
$$

где $\|\cdot\|$ - норма (4.41), постоянная $c_{\delta}$ не зависит от $\varepsilon \in(0,1)$ и $\delta>0-$ малое число.

Условие $g=0$ введено для упрошения формулы (4.44), но его нетрудно соблюсти за счет подходящего продолжения правой части краевого условия (4.3) на $\Omega(\varepsilon)$. Сформулированные здесь результаты получены в [30] при менее ограничительных предположениях, в частности, для задач с быстроосциллируюшими коэффициентами. Кроме того, в [30], [45], [106] рассматривались задачи, для которых результируюшая система содержит мальй параметр $\varepsilon^{1 / q}(q>1)$ при старших производных (типичная ситуация в теории оболочек); в [106] найдена весовая норма | · в неравенстве Корна (4.40), позволяющая вывести аналогичную (4.45) оценку.

ПримеР 4.9. 1) В задаче о деформации упругой пластины $s_{1}=1, s_{2}=1, m=1$ и $T^{1}=J^{1}=2$, причем равенства (4.43) верны в силу (4.32). Формула (4.33) восстанавливает все жесткие смешения (1.37) по полиномам $v=\left(v_{1}, v_{2}, v_{3}\right)$ из линеала (1.42), отвечающего результируюшей системе. Неравенство Корна (4.40) с $t_{1}=t_{2}=t_{3}=1$ и $h_{1}=h_{2}=1, h_{3}=2$ доказано в [37] (см. также [107], [45]). Прием из примера 1.13 позволяет приспособить это неравенство и к задачам электроупругости.

2) Сформулированные требования не выполняются для упругого стержня, так как в (4.31) начальные элементы $p^{1,0}=\Phi^{1,0}$ и $p^{2,0}=\Phi^{2,0}$ квазищепочек с длинами 2 имеют ненулевыми те же компоненты, что и полином $p^{4,0}(\eta)=\Phi^{4,0}(\eta)$, возглавляющий цепочку с полудлиной 1. Неравенство Корна (4.40) с весовой нормой (4.41), содержашей показатели $t_{1}=t_{2}=t_{3}$ и $h_{1}=h_{2}=2, h_{3}=1$, проверено в [37], однако обоснование теории тонких стержней удалось провести в [55] лишь при "улучшении" нормы (4.41), полученном при учете результатов [34], [108]. Помимо интегральных в [55] вьведены поточечные (в классах Гёльдера) оценки разности истинного решения и его асимптотического приближения, найденного в рамках одномерной модели, но дополненного пограничными слоями.

\section{§. Пограничные слои для тонких областей и их сочленений}

1. Обсуждение. Процедура построения асимптотики, описанная в предыдушем параграфе, позволяет в принципе построить асимптотический ряд для решения $u(\varepsilon, y, z)$ задачи $(4.2)-(4.4)$

$$
\varepsilon^{0} \mathscr{U}^{0}\left(\varepsilon^{-1} y, z\right)+\varepsilon^{1} \mathscr{U}^{1}\left(\varepsilon^{-1} y, z\right)+\cdots+\varepsilon^{k} \mathscr{U}^{k}\left(\varepsilon^{-1} y, z\right)+\cdots
$$

где $\mathscr{U}^{k}(\eta, z)$ - конгломерат $(4.21)$, содержаший решения $v^{k}$ и $U^{k}$ результируюшей (4.23) , (4.24) и предельной (4.9) задач. Ряд (5.1) формально удовлетворяет соотношениям (4.2) и (4.3), однако его нельзя рассматривать как приближенное решение задачи в $\Omega(\varepsilon)$. Дело в том, что условие Дирихле (4.4) на торце $\Gamma(\varepsilon)$ может быть выполнено 
разве лишь случайно: постановка неоднородных краевых условий (4.24) для результируюшей системы $\mathfrak{M}\left(\nabla_{z}\right) v^{k}=0$ в $S$ компенсирует в (4.4) невязки специального вида, но слагаемое $U^{k}$ в $\mathscr{U}^{k}$ может быть произвольно. Как известно (см. [109]-[114], [44], $[43 ;$ гл. 15,16$]$ и др.), полное устранение невязок на торцевых поверхностях осушествляется посредством построения пограничного слоя. Соответствующая предельная краевая задача в полуцилиндре $\mathbb{Q}_{+}=\omega \times \mathbb{R}_{+}$возникает после перехода к $\varepsilon=0$ в задаче (4.2)-(4.4), записанной при помощи быстрых переменных

$$
\eta=\varepsilon^{-1} y, \quad \zeta=-\varepsilon^{-1} \nu
$$

где $|\nu|=\operatorname{dist}\{z, \partial S\}$ и $\nu>0$ вне $S$. Имея в виду запросы теории осреднения краевых задач с быстроосциллирующими коэффициентами (см. [47]-[51] и др.), в следующем разделе мы включаем в рассмотрение дифференциальные операторы с периодическими коэффициентами и квазицилиндры, имеющие переменное (также периодическое) сечение.

Другие геометрические формы, связанные с явлением пограничного слоя, возникают при изучении сочленений сингулярно вырождающихся областей с различными предельными размерностями (см. [115]-[125] и др.). В п. 3 рассматриваются краевые задачи на объединении полупространства $\mathbb{R}_{+}^{n}$ и полуцилиндра $\mathbb{Q}_{-}=\omega \times(-\infty, 0]$. При этом допускаются дифференциальные операторы с кусочно постоянными коэффициентами, требующие постановки естественных условий сопряжения (трансмиссии) на поверхности $\omega(0)=\partial \mathbb{R}_{+}^{n} \cap \partial \mathbb{Q}_{-}$.

В этом параграфе рассматриваются системы уравнений второго порядка. Такое ограничение позволяет максимально упростить формулировки всех утверждений. Разумеется, оно не является принципиальным и при помощи тех же соображений, что и в $\S 2$ и $\S 3$, теоремы без особого труда приспосабливаются к произвольным операторам-матрицам $\mathscr{L}\left(x, \nabla_{x}\right)$ с простой или (при обычных дополнительных предположениях) усложненной структурой. Последнее демонстрируется на примере системы Стокса.

2. Квазицилиндрические области. Пусть $S$ - ячейка периодичности, порождаюшая квазищилиндр $\mathbb{Q}$ с периодически изменяюшимся сечением $\omega(t)=\{x \in \mathbb{Q}$ : $z=t\}$. Иными словами, ограниченная область $S$ расположена в слое $\{x=(y, z)$ : $\left.y \in \mathbb{R}^{n-1}, 0<z<1\right\}$ и замыкание $\overline{\mathbb{Q}}$ совпадает с объединением сдвигов ячейки $\bar{S}_{m}=\{x:(y, z-m) \in \bar{S}\}, m \in\{0, \pm 1, \ldots\}$. Предполагаем, что $\mathbb{Q}$ - область с гладкой $(n-1)$-мерной границей $\partial \mathbb{Q}$. Матрицу-функцию $A$ из формулы $(1.10)$ для $\mathscr{L}\left(x, \nabla_{x}\right)$ считаем гладкой и дополнительно 1-периодической по переменной $z$. Элементы $\Lambda_{h k}\left(\nabla_{x}\right)$ алгебраически комплектной матрицы $\Lambda\left(\nabla_{x}\right)$ из той же формулы суть дифференциальные операторы первого порядка с постоянными коэффициентами, т.е. $\operatorname{ord} \mathscr{L}_{j k}=2$ и $t_{1}=\cdots=t_{T}=1$. В силу предложений 1.5 и 1.6 соответствующая квадратичная форма (1.11) обладает полиномиальными свойствами $1^{0}-4^{0}$ с линеалом $\mathscr{P}$.

На множестве $\mathbb{Q}_{+}=\{x \in \mathbb{Q}: z>0\}$ с боковой поверхностью $\Sigma=\partial \mathbb{Q} \backslash \overline{\omega(0)}$ рассмотрим краевую задачу

$$
\begin{aligned}
\mathscr{L}\left(x, \nabla_{x}\right) u(x) & =f(x), \quad x \in \Omega \equiv \mathbb{Q}_{+}, \\
\mathscr{B}\left(x, \nabla_{x}\right) u(x) & =g(x), \quad x \in \Sigma, \\
\mathscr{B}^{\#}\left(x, \nabla_{x}\right) u(x) & =g^{\#}(x), \quad x \in \Sigma^{\#} \equiv \omega(0) .
\end{aligned}
$$


Граничные операторы $\mathscr{B}$ и $\mathscr{B} \#$ составлены по правилу (1.6), но способы отбора строк матрищ $\mathscr{D}=\mathbb{I}_{T}$ и $\mathscr{N}($ см. (1.13)) могут различаться для боковой поверхности $\Sigma$ и торца $\Sigma^{\#}$. Порядки элементов $\mathscr{B}_{j k}$ и $\mathscr{B}_{j k}^{\#}$ обозначаем $r_{j}+t_{k}$ и $r_{j}^{\#}+t_{k}$ соответственно; так как $t_{k}=1$, имеем $r_{j} \leqslant 0$ и $r_{j}^{\#} \leqslant 0$. Под $\mathscr{T}$ и $\mathscr{T}_{\#}^{\#}$ подразумеваем парные с $\mathscr{B}$ и $\mathscr{B} \#$ операторы в формуле Грина вида (1.7).

Граница $\partial \mathbb{Q}_{+}$имеет две особенности - бесконечно удаленную точку и ребро $\Upsilon=\partial \omega(0)$ размерностью $d=n-2$ (требования из п. $2 \S 2$ обеспечиваются следующим условием: для любой точки $x^{0} \in \Upsilon$ раствор двугранного угла, образованного $\Pi^{\#}=\{x: z=0\}$ и гиперплоскостью $\Pi\left(x^{0}\right)$, касательной к $\partial \mathbb{Q}$ в $x^{0}$, принадлежит интервалу $(0, \pi))$. Каждая из названных особенностей привносит в норму свой весовой множитель, экспоненциальный и степенной соответственно. Положим $\rho_{0}(x)=\min \{1, \operatorname{dist}(x, \Upsilon)\}$ и при $l \geqslant 0, \beta, \gamma \in \mathbb{R}$ введем пространство $W_{\beta, \gamma}^{l}(\Omega, \Upsilon)$ как пополнение $C_{0}^{\infty}(\bar{\Omega} \backslash \Upsilon)$ по норме

$$
\left\|w ; \mathscr{W}_{\beta, \gamma}^{l}(\Omega, \Upsilon)\right\|=\left(\sum_{k=0}^{l}\left\|\rho_{0}^{\beta-l+k} \exp (\gamma|z|) \nabla_{x}^{k} w ; L_{2}(\Omega)\right\|^{2}\right)^{1 / 2} .
$$

Рассмотрим оператор $\mathscr{A}_{\beta, \gamma}^{l}$ задачи (5.2)-(5.4),

$$
\begin{aligned}
& \mathscr{W}_{\beta, \gamma}^{l+1}(\Omega)^{T}=\mathscr{D}_{\beta, \gamma}^{l} \mathscr{W}(\Omega) \ni u \mapsto \mathscr{A}_{\beta, \gamma}^{l} u=\left(\mathscr{L} u, \mathscr{B} u, \mathscr{B}^{\#} u\right) \\
& \quad=\left(f, g, g^{\#}\right) \in \mathscr{R}_{\beta, \gamma}^{l} \mathscr{W}(\Omega)=\mathscr{W}_{\beta, \gamma}^{l-1}(\Omega)^{T} \times \mathscr{W}_{\beta, \gamma}^{l-\mathbf{r}-1 / 2}(\Sigma) \times \mathscr{W}_{\beta, \gamma}^{l-\mathbf{r}^{\#}-1 / 2}\left(\Sigma^{\prime}\right)
\end{aligned}
$$

Нормы в следовых пространствах $\mathscr{W}_{\beta, \gamma}^{l-1 / 2}(\Sigma)$ и т.п. вводятся подобно (2.4), а под $\mathscr{W}_{\beta, \gamma}^{l-\mathbf{r}-1 / 2}(\Sigma)$ и т. п. подразумеваются аналоги последних произведений в (2.6), причем $\mathbf{r}=\left(r_{1}, \ldots, r_{T}\right)$ и $\mathbf{r}^{\#}=\left(r_{1}^{\#}, \ldots, r_{T}^{\#}\right)$. Ясно, что отображение (5.6) непрерьвно при $l \in\{1,2, \ldots\}$ и всех $\beta, \gamma \in \mathbb{R}$. Ограничения на весовые показатели $\beta$ и $\gamma$, обеспечивающие фредгольмовость оператора $\mathscr{A}_{\beta, \gamma}^{l}$, находятся посредством локальных рассмотрений. Поэтому границы для показателя $\beta$ устанавливаются на основе материала п. $2-4 \S 2$. Кроме того, теория эллиптических задач с периодическими коэффицциентами (см. [94], [95], [126], [127], [31; §3.4, §5.1]) приспосабливает результаты из $\S 3$ к рассматриваемой задаче и доставляет допустимые значения индекса $\gamma$. Наконец, полиномиальное свойство используется обычным способом. Для окончательных формулировок понадобится ряд обозначений.

Введем подпространства полиномов

$$
\begin{aligned}
P(\mathscr{B}) & =\{p \in \mathscr{P}: \mathscr{B} p=0 \text { на } \Sigma\}, \\
\mathscr{P}\left(\mathscr{B}, \mathscr{B}^{\#}\right) & =\left\{p \in P(\mathscr{B}): \mathscr{B}_{p}^{\#}=0 \text { на } \Sigma^{\#}\right\}, \\
P_{0}\left(x^{0}\right) & =\left\{p \in \mathscr{P}\left(\mathscr{B}, \mathscr{B}^{\prime}\right): p=\mathrm{const}\right\}, \quad x^{0} \in \Upsilon .
\end{aligned}
$$

Линеал $P(\mathscr{B})$ играет роль линеала $P^{\mu}\left(B^{\mu}\right)$ из предложения 3.2 - он будет присоединен к пространству $\mathscr{D}_{\beta, \gamma}^{l} \mathscr{W}(\Omega)$ при обработке асимптотики на бесконечности. Подпространство $\mathscr{P}\left(\mathscr{B}, \mathscr{B}^{\prime}\right)$ содержит все решения однородной задачи $(5.2)-(5.4)$ с конечной 
энергией $a(u, u ; \Omega)$. Наконец, $P_{0}\left(x^{0}\right)$ - линеал (2.33), обслуживаюший модельную задачу в угле $\mathbb{K}^{0}$ (двумерном конусе; см. п. $2 \S 2$ ) и отделяемьй в асимптотике $(2.45)$ решения вблизи ребра. Ясно, что $P_{0}=P_{0}\left(x^{0}\right)$ не зависит от точки $x^{0}$, т.е. заведомо обладает свойствами, постулированными в п. $4 \S 2$. Видимые упрощения в определениях (5.7) стали возможньми благодаря следующим фактам: во-первых, согласно предложению 1.6 линеал $\mathscr{P}$ сохраняется при замораживании коэффициентов, во-вторых, в (2.33) полиномы обобщенной степени $\operatorname{deg}\{p ; \mathbf{t}\}=-(n-d) / 2$ оказьваются постоянными (так как $t_{j}=1$ и $d=n-2$ ) и, в-третьих, в качестве граничного оператора Дирихле взята единичная матрища $\mathbb{I}_{T}$. Если воспользоваться приемом (1.38) и заменить $\mathbb{I}_{T}$ произвольной унитарной матрищей-функцией $\mathscr{U}$, то линеалы $P\left(x^{0}\right)$, вообще говоря, могут приобрести зависимость от $x^{0}$.

Далее считаем, что

$$
l \geqslant 1, \quad \gamma \in(0, \delta), \quad \beta \in\left(l-\delta^{0}, l\right) .
$$

Аналогично (2.45) и (3.10) составим пространство $\mathbf{D}_{\beta, \gamma}^{l} \mathscr{W}(\Omega)$ из функций, допускающих представление

$$
u=\mathfrak{C} k+\chi_{\infty} p+\tilde{u},
$$

где $\tilde{u} \in \mathscr{W}_{\beta, \gamma}^{l+1}(\Omega)^{T}, p \in P(\mathscr{B})$ и $k$ - (векторная) функция из класса $H^{l-\beta}(\Upsilon)^{T}$ со значениями в $P_{0}$. Кроме того, в (5.9) $\mathfrak{C}$ - оператор продолжения с ребра $\Upsilon$ на область $\Omega=\mathbb{Q}_{+}$, введенньй в п. $4 \S 2$, а $\chi_{\infty} \in C^{\infty}(\mathbb{R})-$ срезающая функция, $\chi_{\infty}(z)=1$ при $z>2$ и $\chi_{\infty}(z)=0$ при $z<1$. По аналогии с $\S 2$ и $\S 3$ норма в $\mathbf{D}_{\beta, \gamma}^{l} \mathscr{W}(\Omega)$ определяется естественным образом при отождествлении этого пространства с прямым произведением

$$
H^{l-\beta}(\Upsilon)^{\operatorname{dim} P_{0}} \times \mathbb{C}^{\operatorname{dim} P(\mathscr{B})} \times \mathscr{W}_{\beta, \gamma}^{l+1}(\Omega)^{T} \ni(k, p, \tilde{u}) .
$$

Решения $u \in \mathbf{D}_{\beta, \gamma}^{l} \mathscr{W}(\Omega)$ характерны тем, то для них конечен интеграл энергии $a\left(u, u ; \mathbb{Q}_{+}\right)$. Разумеется, сложное описание $(5.9),(5.10)$ пространства упрощается в конкретных ситуациях, когда первые сомножители из (5.10) большей частью исчезают.

ТЕОрема 5.1. Положительные $\delta$ и $\delta^{0}$ можно выбрать так, чтоби при ограничениях (5.8) оператор задачи (5.2)-(5.4),

$$
\mathbf{A}_{\beta, \gamma}^{l}=\left\{\mathscr{L}, \mathscr{B}, \mathscr{B}^{\#}\right\}: \mathbf{D}_{\beta, \gamma}^{l} \mathscr{W}(\Omega) \rightarrow \mathscr{R}_{\beta, \gamma}^{l} \mathscr{W}(\Omega),
$$

стал фредгольмовым с нулевым индексом. При этом $\operatorname{ker} \mathbf{A}_{\beta, \gamma}^{l}=\mathscr{P}\left(\mathscr{B}, \mathscr{B}^{\#}\right)$, а условиями существования решения $u \in \mathbf{D}_{\beta, \gamma}^{l} \mathscr{W}(\Omega)$ задачи с правой частью $\left(f, g, g^{\#}\right) \in \mathscr{R}_{\beta, \gamma}^{l} \mathscr{W}(\Omega)$ служст равенства

$$
(f, p)_{\Omega}+(g, \mathscr{T} p)_{\Sigma}+\left(g^{\#}, \mathscr{T}^{\#} p\right)_{\Sigma^{\prime}}=0 \quad \forall p \in \mathscr{P}\left(\mathscr{B}, \mathscr{B}^{\#}\right) .
$$

В случае краевых условий Неймана $\mathscr{B}=\mathscr{N}, \mathscr{B} \#=\mathscr{N}$ можно использовать пространство $\mathscr{W}_{\beta, \gamma}^{l+1,0}(\Omega)$ со ступенчатой весовой нормой

$$
\left\|w ; \mathscr{W}_{\beta, \gamma}^{l+1,0}(\Omega, \Upsilon)\right\|=\left(\sum_{k=0}^{l+1}\left\|\rho_{0}^{\beta-l-1+k+\delta_{k, 0}} \exp (\gamma|z|) \nabla_{x}^{k} w ; L_{2}(\Omega)\right\|^{2}\right)^{1 / 2}
$$

(поскольку $s=0$, в (2.38) $\Theta(s-k)$ заменяется на $\left.\delta_{k, 0}\right)$. Связь между пространствами $\mathscr{W}_{\beta, \gamma}^{l+1,0}(\Omega)^{T}$ и $\mathbf{D}_{\beta, \gamma}^{l} \mathscr{W}(\Omega)$ обнаруживается при помощи леммы 2.16 . 
СлеДСТвИЕ 5.2. При ограничениях (5.8) с малыми положительными $\delta$ и $\delta^{0}$ оператор задачи Неймана

$$
\{\mathscr{L}, \mathscr{N}\}: \mathscr{W}_{\beta, \gamma}^{l+1,0}(\Omega, \Upsilon)^{T} \rightarrow \mathscr{W}_{\beta, \gamma}^{l-1}(\Omega, \Upsilon)^{T} \times \mathscr{W}_{\omega, \gamma}^{l-1 / 2}(\partial \Omega, \Upsilon)^{T}
$$

оказывается фредгольмовым мономорфизмом, причем условия разрешимости задачи принимают вид (3.14).

При постановке условий Дирихле хотя бы на части границы $\partial \Omega$ многие из подпространств полиномов становятся тривиальными (ср. с замечанием 2.15).

СлЕДСТвИЕ 5.3. Пусть $\mathscr{B}=\mathscr{D}$ на боковой поверхности $\Sigma$ и, значит, $\mathbf{r}=0$. Тогда оператор (5.6) задачи (5.2)-(5.4) оказывается изоморфизмом при

$$
l \geqslant 1, \quad|\gamma|<\delta, \quad|\beta-l|<\delta^{0} .
$$

СлеДСтвИе 5.4. Если $\mathscr{B} \#=\mathscr{D}^{\#}$ на $\Sigma^{\#} u \mathbf{r}^{\#}=0, m o \operatorname{dim} P\left(x^{0}\right)=0, k=0$ в (5.9), а задача (5.2)-(5.4) с правой частью $\left(f, g, g^{\#}\right) \in \mathscr{R}_{\beta, \gamma}^{l} \mathscr{W}(\Omega)$, əде

$$
l \geqslant 1, \quad \gamma \in(0, \delta), \quad|\beta-l|<\delta^{0},
$$

имеет единственное решение, представимое в виде $u=\chi_{\infty} p+\tilde{u}$, причем $p \in$ $P(\mathscr{B})$ и и $\in \mathscr{W}_{\beta, \gamma}^{l+1}(\Omega)^{T}$. Иными словами, задаче с условиями Дирихле на торие $\Sigma^{\#}$ сопоставлены операторы $\mathbf{A}_{\beta, \gamma}^{l}$ из (5.11) и $\mathscr{A}_{\beta, \gamma}^{l}$ из (5.6), которые являются соответственно изоморфизмом при условии (5.8) и фредгольмовым мономорфизмом при условии (5.15); наконеи, $\operatorname{dim} \operatorname{coker} \mathscr{A}_{\beta, \gamma}^{l}=\operatorname{dim} P(\mathscr{B})$.

ЗАМЕчАнИЕ 5.5. В теории осреднения (см. [47]-[51] и др.) пограничный слой зачастую описывается решениями задач в призме $\Omega=\left\{x=\left(x^{\prime}, x_{n}\right) \in \omega \times \mathbb{R}: x_{n}>\right.$ $\left.F\left(x^{\prime}\right)\right\}$ с криволинейным основанием $\Sigma^{\prime}$; здесь $\omega=\left\{x^{\prime}:\left|x_{j}\right|<h_{j}\right\}$ и $F$ - гладкая функция, $2 h_{j}$-периодическая относительно $x_{j}, j=1, \ldots, n-1$. На противоположных гранях выставляются условия периодичности, делаюшие все ребра на $\partial \Omega$ фиктивными: задачу можно решать в классе $\mathfrak{W}_{\gamma}^{l}(\Omega)$, полученном пополнением по норме $\left\|\exp \left(\gamma x_{n}\right) w ; H^{l}(\Omega)\right\|$ подпространства $2 h_{j}$-периодических функций из $C_{0}^{\infty}(\bar{\Omega})$. В линеале $P^{\omega}$, играюшем роль $\mathscr{P}(\mathscr{B})$, сохраняются те полиномы из $\mathscr{P}$, которые зависят только от переменной $x_{n}$ (в задачах теории упругости $P^{\omega}$ состоит из постоянных векторов). Предыдущие рассуждения применимы и к "периодической" задаче (5.2), (5.4) с правой частью

$$
\left(f, g^{\#}\right) \in \mathfrak{W}_{\gamma}^{l-1}(\Omega)^{T} \times \prod_{q=1}^{T} \mathfrak{H}^{l-r_{q}+1 / 2}\left(\Sigma^{\#}\right),
$$

где $l \geqslant 1, \gamma \in(0, \delta)$, а $\mathfrak{H}^{s}\left(\Sigma^{\#}\right)$ - подпространство $2 h_{j}$-периодических функций из $H^{s}\left(\Sigma^{\#}\right)$. Названная задача имеет решение $u$, представимое в виде суммы $\tilde{u}+\tilde{p} \in$ $\mathfrak{W}_{\gamma}^{l+1}(\Omega)^{T}+P^{\omega}$, тогда и только тогда, когда

$$
(f, p)_{\Omega}+\left(g^{\#}, \mathscr{T}^{\#} p\right)_{\Sigma^{\prime}}=0 \quad \forall p \in P^{\omega}\left(\mathscr{B}^{\#}\right)=\left\{p \in P^{\omega}: \mathscr{B}^{\#} p=0 \text { на } \Sigma^{\#}\right\} .
$$

Само решение определено с точностью до слагаемого из $P^{\omega}(\mathscr{B} \#)$. 
ПримеР 5.6. Приведем результаты для трехмерной системы Стокса, не обслуживаемой теоремой 5.1 по формальным причинам.

1) При постановке условий прилипания

$$
u(x)=0, \quad x \in \partial \Omega,
$$

единственным полиномиальньм решением однородных уравнений Стокса остается $(0,0,0, c)$ (постоянное давление). Поэтому условия существования решения $\mathbf{u}=$ $\left(u, u^{\prime}\right) \in \mathscr{W}_{\beta, \gamma}^{l+1}(\Omega, \Upsilon)^{3} \times \mathscr{W}_{\beta, \gamma}^{l}(\Omega, \Upsilon)$ задачи $(1.45),(1.46)$, (5.16) с правой частью $\left(f, f^{\prime}\right) \in \mathscr{W}_{\beta, \gamma}^{l-1}(\Omega, \Upsilon)^{3} \times \mathscr{W}_{\beta, \gamma}^{l}(\Omega, \Upsilon)$ (индексы подчинены (5.15)) приобретают вид $\left(f^{\prime}, 1\right)_{\Omega}=0$. В указанном классе экспоненциально затухающих вектор-функций решение $\mathbf{u}$ единственно.

2) Рассмотрим систему $(1.45),(1.46)$ с условиями периодичности в призме $\Omega$, описанной в замечании 5.5, и поставим на основании краевое условие

$$
u(x)=g(x), \quad x \in \Sigma^{\prime} .
$$

Как и в примере 3.8 (2), сформулированная задача не обладает полиномиальным свойством в полном объеме, т.е. жордановы цепочки соответствующего пучка не описьваются предложением 3.2 (2). Впрочем, базис в линеале экспоненциальных решений (3.5), отвечаюших $\lambda=0$, находится прямыми вычислениями,

$$
\mathscr{U}^{i, 0}=\mathbf{e}^{i}, \quad \mathscr{U}^{i, 1}(x)=x_{3} \mathbf{e}^{i} \quad(i=1,2), \quad \mathscr{U}^{3,0}=\mathbf{e}^{3}, \quad \mathscr{U}^{4,0}=\mathbf{e}^{4} ;
$$

здесь $\mathbf{e}^{j}=\left(\delta_{j, 1}, \ldots, \delta_{j, 4}\right)$. Повторяя обычные рассуждения, заключаем, что задача (1.45), (1.46), (5.17) с правой частью

$$
\left(f, f^{\prime}, g\right) \in \mathfrak{W}_{\beta, \gamma}^{l-1}(\Omega)^{3} \times \mathfrak{W}_{\beta, \gamma}^{l}(\Omega) \times \mathfrak{H}^{l+3 / 2}\left(\Sigma^{\#}\right)^{3}
$$

(индексы подчинены $(5.15))$ имеет единственное решение в пространстве $\left(\mathfrak{W}_{\beta, \gamma}^{l+1}(\Omega) \dot{+} \mathbb{R}\right)^{3}$ $\times \mathbf{W}_{\beta, \gamma}^{l}(\Omega)$. Это решение убывает при $x_{s} \rightarrow+\infty$ с экспоненщиальной скоростью при вьполнении трех условий ортогональности, устраняющих постоянное слагаемое из вектора скоростей,

$$
\begin{gathered}
\left(f, Z^{i}\right)_{\Omega}+\left(f^{\prime}, Z^{i \prime}\right)_{\Omega}-\left(g, \nu Z^{i \prime}+\partial_{\nu} Z^{i}\right)_{\Sigma^{\prime}}=0, \quad i=1,2 \\
\left(f^{\prime}, 1\right)_{\Omega}-(g, \nu)=0
\end{gathered}
$$

Вектор $\mathbf{Z}^{i}=\left(Z^{i}, Z^{i \prime}\right)$ из (5.18) удовлетворяет однородной задаче $(1.45),(1.46),(5.17)$ и может быть представлен в виде $\mathscr{U}^{i, 1}+\mathfrak{Z}^{i}$, где $\mathfrak{Z}^{i}$ - решение указанного вьше типа при $f=0, f^{\prime}=0$ и $g(x)=-\mathbf{e}^{i} x_{3}$. 
ЗАМЕЧАНИЕ 5.7. В примере 5.6 (2) продемонстрирован обший способ вывода условий экспоненциального затухания решения задачи (5.2)-(5.4). Именно, функции $p$ из левой части (5.12) следует разрешить быть не только полиномом, но и любым решением однородной задачи (5.2)-(5.4), растушим при $x_{n} \rightarrow+\infty$ не быстрее степени $x_{n}$. Упомянутые решения $Z$ ищутся как суммы $\mathscr{U}+\mathfrak{Z}$, где $\mathscr{U}-$ экспоненциальное решение (3.5) при $\lambda=0$ (или аналогичное решение Флоке в случае квазицилиндра) и $\mathfrak{Z} \in \mathbf{D}_{\beta, \gamma}^{l} \mathscr{W}(\Omega)$ - решение задачи (5.2)-(5.4) с $f=0, g=0$ и $g^{\prime}=-\mathscr{U}$. Для сушествования и нетривиальности $Z$ нужно дополнительно потребовать, чтобы $\mathscr{U} \notin \mathscr{P}(\mathscr{B})$ И

$$
0=q(\mathscr{U}, p) \equiv(\mathscr{N} \mathscr{U}, \mathscr{D} p)_{\omega(t)}-(\mathscr{D} \mathscr{U}, \mathscr{N} p)_{\omega(t)} \quad \forall p \in \mathscr{P}(\mathscr{B})
$$

(сечение $\omega(t)$ произвольно). Возникшая антилинейная форма $q$ подобна той, которая фигурировала в (2.60). В силу предложения 1.5 вычитаемое в (5.19) равно нулю.

3. Сочленение полупространства и усеченного цилиндра. Пусть $\Omega=$ $\mathbb{R}_{+}^{n} \cup \mathbb{Q}$, где $\mathbb{Q}$ - цилиндр $\left\{\left(y^{-}, z^{-}\right): y^{-} \in \omega\right\}$ с сечением $\omega \subset \mathbb{R}^{n-1}$, имеюшим компактное замыкание и гладкую границу. Будем считать, что ось $z^{-}$цилиндра не параллельна границе полупространства и положим

$$
\begin{gathered}
\Omega_{+}=\mathbb{R}_{+}^{n}, \quad \Sigma_{+}=\partial \mathbb{R}_{+}^{n} \backslash \overline{\mathbb{Q}} ; \quad \Gamma=\mathbb{Q} \cap \partial \mathbb{R}_{+}^{n}=\partial \mathbb{R}_{+}^{n} \backslash \bar{\Sigma}_{+} \\
\Omega_{-}=\mathbb{Q} \backslash \overline{\mathbb{R}_{+}^{n}}, \quad \Sigma_{-}=\partial \mathbb{Q} \backslash \overline{\mathbb{R}_{+}^{n}} ; \quad \Upsilon=\partial \mathbb{Q} \cap \partial \mathbb{R}_{+}^{n}
\end{gathered}
$$

Дифференциальные уравнения

$$
\mathscr{L}^{ \pm}\left(\nabla_{x}\right) u^{ \pm}(x)=f^{ \pm}(x), \quad x \in \Omega_{ \pm},
$$

и краевые условия

$$
\mathscr{B}^{ \pm}\left(x, \nabla_{x}\right) u^{ \pm}(x)=g^{ \pm}(x), \quad x \in \Sigma_{ \pm},
$$

дополним естественными условиями сопряжения (трансмиссии)

$$
u^{+}(x)=x^{-}(x), \mathscr{N}^{+}\left(x, \nabla_{x}\right) u^{+}(x)-\mathscr{N}^{-}\left(x, \nabla_{x}\right) u^{-}(x)=g^{0}(x), \quad x \in \Gamma .
$$

Под $u^{ \pm}$и т.п. подразумеваем сужение функции $u$ и т.п. на $\Omega_{ \pm}$. Точно так же $\mathscr{L}-$ оператор (1.10) с кусочно постоянной матрицей $A$, совпадаюшей с $A^{ \pm}$на $\Omega_{ \pm}$; порядки $\operatorname{ord} \mathscr{L}_{j k}$ элементов равны двум. Граничные операторы $\mathscr{B}^{ \pm}$построены по правилу (1.6) из операторов $\mathscr{N}^{ \pm}$и $\mathscr{D}^{ \pm}=\mathbb{I}_{T}$, но, вообще говоря, разными способами на $\Sigma_{ \pm} ; \mathscr{N}^{ \pm}$ заданы формулой (1.13) с $A=A^{ \pm}$.

На границе $\partial \Omega$ расположены особенности трех типов: ребро $\Upsilon$ и два выхода на бесконечность, конический $\Omega_{+}$и цилиндрический $\Omega_{-}$. Соответственно в нормы функциональных пространств будут помешены три весовых множителя $\rho_{0}(x)=$ $\min \{1, \operatorname{dist}(x, \Upsilon)\}$ и $\rho_{+}(x)=\max \{1,|x|\}, \rho_{-}(x)=\exp \left(z^{-}\right)$. Норма в пространстве $\mathscr{W}_{\beta, \gamma}^{l}\left(\Omega_{-}, \Upsilon\right)$ уже была введена в (5.5) (обрашаем внимание на показатель $\gamma|z|=$ $-\gamma z^{-} \cos \left(z, z^{-}\right)$в экспоненте). Определим пространство $\mathscr{V}_{\beta, \sigma}^{l}\left(\Omega_{+}, \Upsilon\right)$ как пополнение $C_{0}^{\infty}\left(\bar{\Omega}_{+} \backslash \Upsilon\right)$ по норме

$$
\left\|v ; \mathscr{V}_{\beta, \sigma}^{l}\left(\Omega_{+}, \Upsilon\right)\right\|=\left(\sum_{k=0}^{l}\left\|\rho_{0}^{\beta-l+k} \rho_{+}^{\sigma-l+k} \nabla_{x} v ; L_{2}\left(\Omega_{+}\right)\right\|^{2}\right)^{1 / 2} .
$$


Подчеркнем, что индексы $\beta$ в (5.5) и (5.23) одинаковы.

Составим пространство $\mathscr{D}_{\beta, \gamma, \sigma}^{l}\left(\Omega_{ \pm}, \Upsilon\right)$ из таких функций $u$, что их сужения $u^{+} \in \mathscr{V}_{\beta, \sigma}^{l+1}\left(\Omega_{+}\right)^{T}$ и $u^{-} \in \mathscr{W}_{\beta, \gamma}^{l+1}\left(\Omega_{-}\right)^{T}$ совпадают на $\Gamma$. Кроме того, $\left(f, g, g^{0}\right) \in$ $\mathscr{R}_{\beta, \gamma, \sigma}^{l}\left(\Omega_{ \pm}, \Upsilon, \Gamma\right)$, если $g^{0} \in V_{\beta}^{l+1 / 2}(\Gamma)^{T}$, а сужения $\left(f^{+}, g^{+}\right)$и $\left(f^{-}, g^{-}\right)$принадлежат соответственно произведениям

$$
\mathscr{V}_{\beta, \sigma}^{l-1}\left(\Omega_{+}\right)^{T} \times \prod_{q=1}^{T} \mathscr{V}_{\beta, \sigma}^{l-r_{q}^{+}-1 / 2}\left(\Sigma_{+}\right), \quad \mathscr{W}_{\beta, \gamma}^{l-1}\left(\Omega_{-}\right)^{T} \times \prod_{q=1}^{T} \mathscr{W}_{\beta, \gamma}^{l-r_{q}^{-}-1 / 2}\left(\Sigma_{-}\right)
$$

С задачей (5.20)-(5.22) свяжем отображение

$$
\mathscr{A}_{\beta, \gamma, \sigma}^{l}: \mathscr{D}_{\beta, \gamma, \sigma}^{l}\left(\Omega_{ \pm}, \Upsilon\right) \rightarrow \mathscr{R}_{\beta, \gamma, \sigma}^{l}\left(\Omega_{ \pm}, \Upsilon, \Gamma\right),
$$

непрерывное при любых $l \in\{1,2, \ldots\}$ и $\beta, \gamma, \sigma \in \mathbb{R}$.

Введем используемые далее подпространства полиномов

$$
\begin{aligned}
P\left(\mathscr{B}^{ \pm}\right) & =\left\{p \in \mathscr{P}: \mathscr{B}^{ \pm} p=0 \text { на } \Sigma_{ \pm}\right\}, \\
P^{0}\left(\mathscr{B}^{+}\right) & =\left\{p \in P\left(\mathscr{B}^{+}\right): p=\mathrm{const}\right\} \\
P_{0}\left(x^{0}\right) & =P^{0}\left(\mathscr{B}^{+}\right) \cap P\left(\mathscr{B}^{-}\right), \quad x^{0} \in \Upsilon .
\end{aligned}
$$

Отметим, что в силу предложения 1.6 линеал $\mathscr{P}$ одинаков для $\mathscr{L}^{+}$и $\mathscr{L}^{-}$. Остается верньм сказанное в п. 2 о линеале $P_{0} \equiv P_{0}\left(x^{0}\right)$ (см. комментарий к (5.7)). Подпространство $P^{0}\left(\mathscr{B}^{+}\right)$будет востребовано лишш в случае $n=2$.

Пусть индексы $l$ и $\beta, \gamma$ подчинены (5.8). Расширим область определения оператора задачи $(5.20)-(5.22)$ и включим в $\mathbf{D}_{\beta, \gamma, \sigma}^{l}(\Omega)$ функции $u$, допускаюшие представление (5.9), в котором $\tilde{u} \in \mathscr{D}_{\beta, \gamma, \sigma}^{l}(\Omega), p \in P\left(\mathscr{B}_{-}\right), k$ - вектор-функция из класса $H^{l-\beta}(\Upsilon)^{T}$ со значениями в $P_{0}$, а $\chi_{\infty} \in C^{\infty}\left(\mathbb{R}^{n}\right)$ - срезка, $\chi_{\infty}(x)=1$ при $z<-2$ и $\chi_{\infty}(x)=0$ при $z>-1$. Подчеркнем, что элементы пространства $\mathbf{D}_{\beta, \gamma, \sigma}^{l}(\Omega)$ по-прежнему удовлетворяют первому из условий сопряжения (5.22), а значит, оператор задачи (5.20)-(5.22), реализуемый как отображение

$$
\mathbf{A}_{\beta, \gamma, \sigma}^{l}: \mathbf{D}_{\beta, \gamma, \sigma}^{l}\left(\Omega_{ \pm}, \Upsilon\right) \rightarrow \mathscr{R}_{\beta, \gamma, \sigma}^{l}\left(\Omega_{ \pm}, \Upsilon, \Gamma\right),
$$

сохраняет непрерывность при ограничениях (5.8). Более того, появление условий (5.22) никак не сказьвается на применяемой схеме исследования операторов задач, поскольку, как проверено в [128], результаты теории краевых задач в областях с кусочно гладкой гранищей без каких-либо изменений переносятся на задачи с условиями сопряжения (трансмиссии).

Аналогично (5.10) норма в $\mathbf{D}_{\beta, \gamma, \sigma}^{l}\left(\Omega_{ \pm}, \Upsilon\right)$ определяется при отождествлении этого пространства с прямым произведением

$$
H^{l-\beta}(\Upsilon)^{\operatorname{dim} P_{0}} \times \mathbb{C}^{\operatorname{dim} P\left(\mathscr{B}^{-}\right)} \times \mathscr{D}_{\beta, \gamma, \sigma}^{l}\left(\Omega_{ \pm}, \Upsilon\right) \ni(k, p, \tilde{u})
$$


ТЕОрема 5.8. 1) Пусть $n \geqslant 3$, причем выполнень требования (5.8) и

$$
|\sigma-l|<\delta^{+}
$$

где $\delta^{+}=-1+n / 2$. Тогда оператор (5.25) задачи (5.20)-(5.22) есть изоморфизм.

2) Пусть $n=2$, причем выполнены требования (5.8) и

$$
\sigma \in\left(l, l+\delta^{+}\right)
$$

где $\delta^{+}=1$. Задача (5.20)-(5.22) с правой частью $\left(f, g, g^{0}\right) \in \mathscr{R}_{\beta, \gamma, \sigma}^{l}\left(\Omega_{ \pm}, \Upsilon, \Gamma\right)$ имеет единственное решение $u$, представимое в виде $u=\chi_{+} p+\hat{u}$, где $p-$ столбеи из $P^{0}\left(\mathscr{B}^{+}\right) \subset \mathbb{C}^{T}, \hat{u} \in \mathbf{D}_{\beta, \gamma, \sigma}^{l}\left(\Omega_{ \pm}, \Upsilon\right)$ u $\chi_{+} \in C^{\infty}\left(\mathbb{R}^{n}\right)-$ срезка, равная единице в $\mathbb{R}_{+}^{n}$ при $|x|>2 R$ и нулю при $z<-1$ или $|x|<R(R-$ радиус шара в $\mathbb{R}^{n-1}$, содержащего Г). Справедлива оценка

$$
\left\|p ; \mathbb{C}^{T}\right\|+\left\|\hat{u} ; \mathbf{D}_{\beta, \gamma, \sigma}^{l}\left(\Omega_{ \pm}, \Upsilon\right)\right\| \leqslant c\left\|\left(f, g, g^{0}\right) ; \mathscr{R}_{\beta, \gamma, \sigma}^{l}\left(\Omega_{ \pm}, \Upsilon, \Gamma\right)\right\| .
$$

ЗАмЕчАНИЕ 5.9. В (5.26) и (5.27) гранищы изменения весового индекса $\sigma$ установлены в соответствии с предложением 2.21 (2) (в случае $\mathscr{B}^{+}=\mathscr{D}$ они могут быть расширены; см. далее следствия 5.10 и 5.11). Согласно следствию 2.4 для любого конуса $\mathbb{K}^{+} \subset \mathbb{R}^{n}, n \geqslant 3$, сушествует такое $\delta>0$, что полоса $(2.13), \mu=+$, свободна от спектра пучка $\mathfrak{A}^{+}$, порожденного задачей $\left\{\mathscr{L}^{+}, \mathscr{B}^{+}\right\}$в $\mathbb{K}^{+}$. Поэтому теорема 5.8 (1) остается в силе при замене $\mathbb{R}_{+}^{n}$ на $\mathbb{K}^{+}$. Благодаря предложению 2.3 теорема 5.8 (2) сохраняется для сочленения полуполосы $\mathbb{Q}_{-}$с произвольным углом $\mathbb{K}^{+} \subset \mathbb{R}^{2}$, но число $\delta^{+}$в (5.27) может уменьшиться (ср. предложение 2.22).

Уточним теорему 5.8 при постановке на $\Sigma_{+}$или $\Sigma_{-}$условий Дирихле, которые делают тривиальным подпространство $P_{0}$ и тем самым устраняют из (5.9) асимптотический член $\mathfrak{C} k$, отделенный около ребра $\Upsilon$.

СЛЕДСТВИЕ 5.10. Пусть $\mathscr{B}^{-}=\mathscr{D} u \delta^{+}=-1+n / 2$ в случае $\mathscr{B}^{+} \neq \mathscr{D}, n \geqslant 3$ uлu $\delta^{+}=n / 2$ в случае $\mathscr{B}^{+}=\mathscr{D}, n \geqslant 2$. Тогда найдутся такие $\delta^{0}>0$ и $\delta>0$, что при ограничениях (5.14) и (5.26) оператор (5.24) задачи (5.20)-(5.22) - изоморфизм.

СлЕДСТВИЕ 5.11. Пусть $\mathscr{B}^{+}=\mathscr{D} u \delta^{+}=n / 2, n \geqslant 2$. Тогда при условиях (5.26) $u(5.15)$ с некоторыми $\delta, \delta^{0}>0$ задача (5.20)-(5.22) с правой частью $\left(f, g, g^{0}\right) \in$ $\mathscr{R}_{\beta, \gamma, \sigma}^{l}\left(\Omega_{ \pm}, \Upsilon, \Gamma\right)$ имеет единственное решение $и$, представимое в виде

$$
u=\chi_{\infty} p+\tilde{u},
$$

где $\tilde{u} \in \mathscr{D}_{\beta, \gamma, \sigma}^{l}\left(\Omega_{ \pm}, \Upsilon\right), p \in P\left(\mathscr{B}^{-}\right)$, а $\chi_{\infty}-$ срезка, введенная перед (5.25). Справедливо неравенство

$$
\left\|p ; P\left(\mathscr{B}^{-}\right)\right\|+\left\|\tilde{u} ; \mathscr{D}_{\beta, \gamma, \sigma}^{l}\left(\Omega_{ \pm}, \Upsilon\right)\right\| \leqslant c\left\|\left(f, g, g^{0}\right) ; \mathscr{R}_{\beta, \gamma, \sigma}^{l}\left(\Omega_{ \pm}, \Upsilon, \Gamma\right)\right\|,
$$

причем линеал $P\left(\mathscr{B}^{-}\right)$отождествляется $c \mathbb{C}^{\tau_{-}}$, әде $\tau_{-}=\operatorname{dim} P\left(\mathscr{B}^{-}\right)$. Отображение (5.24) - фредгольмов мономорфизм с коядром размерности $\tau_{-}$.

Если $\mathscr{L}$ - оператор с постоянными коэффициентами $\left(A^{+}=A^{-}\right)$, то условия (5.22) не нужны и составные нормы $(5.5),(5.23)$ в $\Omega_{ \pm}$заменяются единой нормой в $\Omega$ с тремя весовыми множителями $\rho_{0}$ и $\rho_{ \pm} ;$соответствуюшее пространство обозначаем $\mathscr{W}_{\beta, \gamma, \sigma}^{l}(\Omega, \Upsilon)$, и это - единственное изменение, требующееся в приведенных утверждениях. Упомянем задачу Стокса, у которой такие же свойства, как и отмеченные в следствии 5.11. 
ПРимеР 5.12. При $n=2,3$ оператор задачи (1.45), (1.46), (5.16) порождает изоморфизм

$$
\mathscr{W}_{\beta, \gamma, \sigma}^{l+1}(\Omega)^{n} \times\left(\mathscr{W}_{\beta, \gamma, \sigma}^{l}(\Omega) \dot{+} \chi_{\infty} \mathbb{R}\right) \rightarrow \mathscr{W}_{\beta, \gamma, \sigma}^{l-1}(\Omega)^{n} \times \mathscr{W}_{\beta, \gamma, \sigma}^{l}(\Omega) \times \mathscr{W}_{\beta, \gamma, \sigma}^{l+3 / 2}(\partial \Omega)
$$

при условиях (5.15) и $|\sigma-l|<n / 2$. Подчеркнем, что постоянное давление отделяется в асимптотику только на цилиндрическом выходе и в отличие от примера 5.6 (1) оно не принадлежит ядру оператора, так как $1 \notin \mathcal{V}_{\beta, \sigma}^{l}\left(\Omega_{+}\right)$при $\sigma>l-n / 2$.

Обратимся к случаю $\mathscr{B}^{-}=\mathscr{D}, \mathscr{B}^{+} \neq \mathscr{D}_{\text {и }} n=2$, не охваченному следствием 5.10 . Пусть $p^{1}, \ldots, p^{\tau_{+}}-$базис в линеале $P^{0}\left(\mathscr{B}^{+}\right) \subset \mathbb{C}^{T}$. Предложение 2.3 указьвает такие степенные решения (2.16) модельной задачи на полуплоскости $\mathbb{R}_{+}^{2}$ :

$$
\mathscr{U}^{j, 0}=p^{j}, \quad \mathscr{U}^{j, 1}(x)=p^{j} \ln |x|+\Phi^{j, 1}(\varphi), \quad j=1, \ldots, \tau_{+} .
$$

Постоянные векторы из (5.30) формируют асимптотику решения $u$, а при помощи логарифмических строятся решения $Z^{j}$ однородной задачи, которые фигурируют в условиях ортогональности, обеспечиваюших затухание решения $u$ в коническом выходе $\Omega_{+}$. Как будет продемонстрировано далее, в плоской задаче Неймана названные группы решений $\mathscr{U}^{j, 0}$ и $\mathscr{U}^{j, 1}$ обмениваются ролями; в частности, $Z^{j}=\mathscr{U}^{j, 0} \equiv \mathbf{e}^{j}$ в $\Omega$. В очередном утверждении для простоты ограничимся рассмотрением условий Неймана на $\Sigma_{+}$, но переход к смешанньм краевьм условиям несложен.

ПРЕДЛОЖЕНИЕ 5.13. Пусть $n=2 u \mathscr{B}^{-}=\mathscr{D}, \mathscr{B}^{+}=\mathscr{N}^{+}$. Существуют такие $\delta^{0}, \delta>0$, что при выполнении (5.14) и (5.27) задача (5.20)-(5.22) имеет единственное решение

$$
u=\chi+p+\tilde{u}
$$

где $р \in \mathbb{C}^{T}$ и $\tilde{u} \in \mathscr{D}_{\beta, \gamma, \sigma}^{l}\left(\Omega_{ \pm}, \Upsilon\right)$, причем верна подобная (5.29) оченка. Решение и принадлежит $\mathscr{D}_{\beta, \gamma, \sigma}^{l}\left(\Omega_{ \pm}, \Upsilon\right)$ (затухает при $\left.\gamma \geqslant 0\right)$ в том и только том случае, ecлu

$$
\left(f, Z^{j}\right)_{\Omega}+\left(g^{+}, Z^{j}\right)_{\Sigma^{+}}-\left(g^{-}, \mathcal{N}^{-} Z^{j}\right)_{\Sigma^{-}}+\left(g^{0}, Z^{j}\right)_{\Gamma}=0, \quad j=1, \ldots, T .
$$

Функиии $Z^{j}=\chi_{+} \mathbf{e}^{j}+\mathscr{Z}^{j}$ удовлетворяют однороднылм соотношениям (5.20)(5.22), т.е. $\mathscr{Z}^{j}$ - решение вида (5.31), компенсирующее невязки слагаемого $\chi_{+} \mathbf{e}^{j}=$ $\chi_{+}\left(\delta_{1, j}, \ldots, \delta_{T, j}\right)$ в уравнении (5.2) на $\Omega_{+}$и в краевом условии (5.21) на $\Sigma_{+}$.

Для задачи Неймана возможно использование ступенчатых весовых норм, обсуждавшихся в п. $3 \S 2$. В (5.13) уже было введено соответствуюшее пространство $\mathscr{W}_{\beta, \gamma}^{l+1,0}\left(\Omega_{-}, \Upsilon\right)$, а норма в $\mathcal{V}_{\beta, \sigma}^{l+1,0}\left(\Omega_{+}, \Upsilon\right)$ получается из (5.23) заменой показателей $\beta-l+k$ и $\sigma-l+k$ на $\beta-l-1+k+\delta_{k, 0}$ и $\sigma-l-1+k(k=0, \ldots, l+1)$. Как и ранее, из этих пространств сооружается область определения $\mathscr{D}_{\beta, \gamma, \sigma}^{l,-1}\left(\Sigma_{ \pm}, \Upsilon\right)$ оператора задачи. 
ПрЕДЛОЖЕНИЕ 5.14. Пусть $\mathscr{B}^{ \pm}=\mathscr{N}^{ \pm} u\left(f, g, g^{0}\right) \in \mathscr{R}_{\beta, \gamma, \sigma}^{l}\left(\Omega_{ \pm}, \Upsilon, \Gamma\right)$. В ограничениях (5.8) на индексы $l$ и $\beta, \gamma$ найдутся такие $\delta^{0}>0$ и $\delta>0$, что справедливы следующие утверждения.

1) При $n \geqslant 3 u|\sigma-l|<-1+n / 2$ задача Неймана (5.20)-(5.22) имеет единственное решение $и$, представимое в виде (5.28), где $p \in P\left(\mathscr{B}^{-}\right), u \tilde{u} \in \mathscr{D}_{\beta, \gamma, \sigma}^{l,-1}\left(\Omega_{ \pm}, \Upsilon\right)$, и удовлетворяющее аналогичному (5.29) неравенству.

2) Eсли $n=2$ и $\sigma \in(l, l+1)$, то условиями существования решения и задачи Неймана (5.20)-(5.22), допускающего представление (5.28) с $p \in P\left(\mathscr{B}^{-}\right)$u $\tilde{u} \in$ $\mathscr{D}_{\beta, \gamma, \sigma}^{l,-1}\left(\Omega_{ \pm}, \Upsilon\right)$, служат равенства

$$
\left(f_{j}, 1\right)_{\Omega}+\left(g_{j}, 1\right)_{\partial \Omega}+\left(g_{j}^{0}, 1\right)_{\Gamma}=0, \quad j=1, \ldots, T .
$$

Такое решение единственно и для него выполняется подобная (5.29) оценка.

В отличие от задачи Стокса (пример 5.12) свойства задач теории упругости, обеспечиваемые предложениями 5.13 и 5.14 , зависят от размерности $n=2,3$. Именно, решения плоских задач затухают в угловом выходе на бесконечность лишь при дополнительных требованиях (5.32) или (5.33). Это обстоятельство находит свое отражение в алгорифме [120] построения асимптотики решений задач на соединениях областей различных предельных размерностей.

\section{СПИСОК ЛИТЕРАТУРЫ}

[1] Agmon S., Douglis A., Nirenberg L. Estimates near the boundary for solutions of elliptic partial differential equations satisfying general boundary conditions. 1, 2 // Comm. Pure Appl. Math. 1959. V. 12. № 4. P. 623-723; 1964. V. 17. №1. P. 35-92.

[2] Солонников В.А. Об общих краевых задачах для систем, эллиптических в смысле А. Дуглиса-Л. Ниренберга. 1, 2 // Изв. АН СССР. Сер. матем. 1964. Т. 28. №3. С. 665-706; // Труды МИАН. 1966. V. 92. Р. 233-297.

[3] Петровский И. Г. Лекции об уравнениях с частными производными. М.: Наука, 1964.

[4] Агранович М. С., Вишик М.И. Эллиптические задачи с параметром и параболические задачи общего вида // УМН. 1964. Т. 19. №3. С. 53-160.

[5] Лионс ЖК.-Л., Мадженес Э. Неоднородные граничные задачи и их приложения. М.: Мир, 1971.

[6] Ройтберг Я.А., Шефтель З. Г. Теорема о гомеоморфизмах для эллиптических систем и ее приложения // Матем. сб. 1969. Т. 78. №3. С. 446-472.

[7] Хермандер Л. Линейные дифференциальные операторы с частными производными. М.: Мир, 1965.

[8] Roitberg Ya. A. Elliptic Boundary Value Problems in the Spaces of Distributions. Dordrecht: Kluwer, 1996.

[9] Соболев С. Л. Некоторые применения функционального анализа в математической физике. М.: Наука, 1988.

[10] Михлин С. Г. Вариационные методы в математической физике. М.: Наука, 1970.

[11] Ладыженская О.А. Краевые задачи математической физики. М.: Наука, 1973.

[12] Nečas J. Les méthodes directes en théorie des équations elliptiques. Paris: Masson, 1967.

[13] Кондратьев В. А. Краевые задачи для эллиптических уравнений в областях с коническими или угловыми точками // Труды ММО. 1967. Т. 16. С. 209-292.

[14] Кондратьев В. А. О гладкости решения задачи Дирихле для эллиптических уравнений второго порядка в кусочно-гладкой области // Дифференц. уравнения. 1970. Т. 6. № 10. C. $1831-1843$.

[15] Мазья В. Г., Пламеневский Б. А. О коэффициентах в асимптотике решений эллиптических краевых задач в области с коническими точками // Math. Nachr. 1977. V. 76. P. 29-60. 
[16] Мазья В. Г., Пламеневский Б. А. Оценки в $L_{p}$ и в классах Гёльдера и принцип Миранда-Агмона для решений эллиптических краевых задач в областях с особыми точками на границе // Math. Nachr. 1978. T. 81. C. 25-82.

[17] Кондратьев В.А., Олейник О.А. Краевые задачи для уравнений с частными производными в негладких областях // УМН. 1983. Т. 38. № 2. С. 3-76.

[18] Grisvard P. Elliptic Problems is Non-Smooth Domains. London: Pitman, 1985.

[19] Dauge M. Elliptic Boundary Value Problems on Corner Domains. Berlin: Springer-Verlag, 1988. (Lecture Notes in Math. V. 1341.)

[20] Назаров С. А., Пламеневский Б. А. Эллиптические задачи в областях с кусочно гладкой границей. М.: Наука, 1991.

[21] Kozlov V.A., Maz'ya V.G., Rossmann J. Elliptic Boundary Value Problems in Domains with Point Singularities. Providence: Amer. Math. Soc., 1997.

[22] Мазья В.Г., Пламеневский Б. А. Об эллиптических краевых задачах в областях с кусочно гладкой границей // Труды симп. по мех. сплошн. сред и родственным пробл. анализа. Т. 1. Тбилиси: Мецниереба, 1973. С. 171-181.

[23] Мазья В. Г., Пламеневский Б. А. Эллиптические краевые задачи на многообразиях с особенностями // Проблемы матем. анализа. Вып. 6. Л.: Изд-во ЛГУ, 1977. С. 85-142.

[24] Мазья В. Г., Пламеневский Б. А. $L_{p}$-оценки решений эллиптических краевых задач в областях с ребрами // Труды ММО. 1978. Т. 37. С. 49-93.

[25] Назаров С. А., Шойхет Б. А. Коэрцитивные оценки в весовых пространствах решений задач теории упругости и теории ползучести в области с двумерной трещиной // Изв. АН АрмССР. Мех. 1983. Т. 36. № 4. С. 12-25.

[26] Назаров С. А. Оценки вблизи ребра решения задачи Неймана для эллиптической системы // Вестник ЛГУ. 1988. №1. С. 37-42.

[27] Назаров С. А., Пламеневский Б. А. Задача Неймана для самосопряженных эллиптических систем в областях с кусочно гладкой границей // Труды ЛМО. 1990. Т. 1. C. $174-211$.

[28] Мазья В. Г., Пламеневский Б. А. Весовые пространства с неоднородными нормами и краевые задачи в областях с коническими точками // Elliptishe Differentialgleichung (Meeting, Rostock, 1977). Rostock: Wilhelm-Pieck-Univ., 1978. P. 161-189.

[29] Назаров С. А. Метод Вишшка-Люстерника для эллиптических краевых задач в областях с коническими точками. 1. Задача в конусе; 3 . Задача с вырождением в конической точке // Сиб. матем. журн. 1981. Т. 22. № 4. С. 142-163; 1984. Т. 25. №6. С. 106-115.

[30] Назаров С. А. Общая схема осреднения самосопряженных эллиптических систем в многомерных областях, в том числе тонких // Алгебра и анализ. 1995. Т. 7. № 5. С. 1-92.

[31] Nazarov S. A., Plamenevsky B.A. Elliptic Problems in Domains with Piecewise Smooth Boundaries. Berlin: Walter de Gruyter, 1994.

[32] Назаров С.А. Самосопряженные эллиптические краевые задачи. Полиномиальное свойство и формально положительные операторы // Проблемы матем. анализа. Вып. 16. СПб: Изд-во СПбГУ, 1997. С. 167-192.

[33] Назаров С. А. Несамосопряженные эллиптические задачи с полиномиальным свойством в областях, имеющих цилиндрические выходы на бесконечность // Зап. науч. семин. ПОМИ. 1997. Т. 249. С. 212-231.

[34] Кондратьев В.А., Олейник О.А. Краевые задачи для системы теории упругости в неограниченных областях. Неравенство Корна // УМН. 1988. Т. 43. № 5. С. 55-98.

[35] Kondratiev V.A., Oleinik O. A. Hardy's and Korn's type inequalities and their applications // Rend. Mat. Appl. (7). 1990. V. 10. P. 641-666.

[36] Solonnikov V. A. Stokes and Navier-Stokes equations in domains with noncompact boundaries // Nonlinear PDE and Their Appl. Collège de France Seminar. 1989. V. 4. P. 240-349.

[37] Назаров С. А. Неравенства Корна, асимптотически точные для тонких областей // Вестник СПбГУ. 1992. № 8. С. 19-24.

[38] Назаров С. А. Весовые неравенства Корна на параболоидальных областях // Матем. заметки. 1997. Т. 6. № 5. С. 751-765.

[39] Ван Дайк М. Методы возмушений в механике жидкости. М.: Мир, 1967. 
[40] Мазья В. Г., Назаров С. А., Пламеневский Б. А. Асимптотика решений эллиптических краевых задач при сингулярных возмущениях области. Тбилиси: Изд-во ТГУ, 1981.

[41] Leguillon D., Sanches-Palencia E. Computation of Singular Solutions in Elliptic Problems and Elasticity. Paris: Masson, 1987.

[42] Ильин А. М. Согласование асимптотических разложений решений краевых задач. М.: Наука, 1989.

[43] Mazja W. G., Nasarow S. A., Plamenewski B. A. Asymptotische Theorie elliptischer Randwertaufgaben in singulär gestörten Gebieten. V. 1, 2. Berlin: Akademie-Verlag, 1990, 1991.

[44] Назаров С. А. Структура решений эллиптических краевых задач в тонких областях // Вестник ЛГУ. 1982. №7. С. 65-68.

[45] Леора С.Н., Назаров С. А., Проскура А. В. Вывод предельных уравнений для эллиптических задач в тонких областях при помощи ЭВМ // Журн. вычисл. матем. и матем. физики. 1986. Т. 26. №7. С. 1032-1048.

[46] Вайнберг М. М., Треногин В. А. Теория ветвления решений нелинейных уравнений. M.: Наука, 1969.

[47] Bensoussan A., Lions J. L., Papanicolaou G. Asymptotic Analysis for Periodic Structures. Amsterdam: North Holland, 1978.

[48] Бахвалов Н. С., Панасенко Г. П. Осреднение процессов в периодических средах. М.: Наука, 1984.

[49] Санчес-Паленсия Э. Неоднородные среды и теория колебаний. М.: Мир, 1984.

[50] Олейник О.А., Иосифьян Г. А., Шамаев А.С. Математические задачи теории сильно неоднородных упругих сред. М.: Изд-во МГУ, 1991.

[51] ЖКиков В. В., Козлов С. М., Олейник О. А. Усреднение диффференциальных операторов. М.: Наука, 1993.

[52] Ройтберг И. Я., Ройтберг Я. А. Формула Грина для общих эллиптических граничных задач для систем структуры Дуглиса-Ниренберга // Докл. РАН. 1998. Т. 359. №6. C. $739-743$.

[53] Лехницкий С. Г. Теория упругости анизотропного тела. М.: Наука, 1977.

[54] Фикера Г. Теоремы существования в теории упругости. М.: Мир, 1972.

[55] Назаров С. А. Обоснование асимптотической теории тонких стержней. Интегральные и поточечные оценки // Проблемы матем. анализа. Вып. 17. СПб: Изд-во СПбГУ, 1997. C. $101-152$.

[56] Партон В.З., Кудрявцев Б.А. Электромагнитоупругость пьезоэлектрических и электропроводных тел. М.: Наука, 1988.

[57] Новацкий В. Теория упругости. М.: Мир, 1975.

[58] Ладыженская О. А. Математические вопросы динамики вязкой несжимаемой жидкости. М.: Наука, 1970.

[59] Novotny A. Viscous multipolar Fluids. Physical background and mathematical theory // Fortschr. Phys. 1992. V. 40. № 5. P. 445-517.

[60] Мазья В. Г., Назаров С. А., Пламеневский Б. А. Эллиптические краевые задачи в областях типа внешности пика // Проблемы матем. анализа. Вып. 9. Л.: Изд-во ЛГУ, 1984. C. $105-148$.

[61] Стернин Б. Ю. Эллиптические и параболические задачи на многообразиях с границей, состоящей из компонент различной размерности // Труды ММО. 1966. Т. 15. С. 346-382.

[62] Партон В.З., Перлин П. И. Методы математической теории упругости. М.: Наука, 1981.

[63] Costabel M., Dauge M. Computation of corner singularities in linear elasticity // Lecture Notes in Pure Appl. Math. 1994. V. 167. P. 59-68.

[64] Назаров С. А., Пламеневский Б. А. Эллиптические задачи с условиями излучения на ребрах границы // Матем. сб. 1992. Т. 183. № 10. С. 13-44.

[65] Назаров С. А., Пламеневский Б. А. Самосопряженные задачи с условиями излучения на ребрах границы // Алгебра и анализ. 1992. Т. 4. № 3. С. 196-225. 
[66] Никишкин В.А. Особенности решений задачи Дирихле для уравнения второго порядка в окрестности ребра // Вестник МГУ. Сер. матем., мех. 1979. № 2. С. 51-62.

[67] Maz'ja V.G., Rosmann J. Über die Asymptotik der Lösungen elliptisher Randwertaufgaben in der Umgebung von Kanten // Math. Nachr. 1988. V. 138. P. 27-53.

[68] Назаров С. А. Самосопряженные расширения оператора задачи Дирихле в весовых функциональных пространствах // Матем. сб. 1988. Т. 137. №2. С. 224-241.

[69] Назаров С. А., Пламеневский Б. А. Самосопряженные эллиптические задачи: операторы рассеяния и поляризации на ребрах границы // Алгебра и анализ. 1994. Т. 6. № 4. C. $157-186$.

[70] Назаров С. А. Фредгольмовость оператора краевой задачи с условиями на ребре границы типа условий Чаплыгина-Жуковского-Кутта // Функц. анализ и его прил. 1997. T. 31. № 3. C. 44-56.

[71] Назаров С. А. Асимптотические условия в точках, самосопряженные расширения операторов и метод сращиваемых разложений // Труды СПбМО. 1996. Т. 5. С. 112-183.

[72] Назаров С. А., Полякова О.Р.К Критерии разрушения, асимптотические условия в вершинах трешин и самосопряженные расширения оператора Ламе // Труды ММО. 1996. T. 57. C. $16-75$.

[73] Nazarov S. A. On the two-dimensional aperture problem for Navier-Stokes equations // C. R. Acad. Sci. Paris. Sér. 1. 1996. V. 323. P. 699-703.

[74] Williams M. L. Stress singularities resulting from various boundary conditions in angular corners of plates in extensions // J. Appl. Mech. 1952. V. 19. № 4. P. 526, 528.

[75] Solonnikov V.A. On the Stokes equations in domains with nonsmooth boundaries and on a viscous incompressible flow with a free surface // Nonlinear PDE and Their Appl. Collège de France Semin. 1980/81. V. 3. P. 340-423.

[76] Мазья В.Г., Назаров С. А., Пламеневский Б. А. Об особенностях решений задачи Дирихле во внешности тонкого конуса // Матем. сб. 1983. Т. 122. № 4. С. 435-456.

[77] Мазья В. Г., Назаров С. А. Об особенностях решений задачи Неймана в конической точке // Сиб. матем. журн. 1989. Т. 30. №3. С. 52-63.

[78] Мовчан Н. В., Назаров С. А. Асимптотика показателей сингулярностей для угловых в плане трешин // Вестник ЛГУ. 1990. № 15. С. 34-38.

[79] Козлов В.А., Мазья В. Г. О спектре операторного пучка, порожденного задачей Дирихле в конусе // Матем. сб. 1991. Т. 182. № 5. С. 638-660.

[80] Козлов В.А., Мазья В. Г. Спектральные свойства операторных пучков, порожденных эллиптическими краевыми задачами в конусе // Функц. анализ и его прил. 1988. Т. 22. № 2. C. 38-46.

[81] Kozlov V.A., Maz'ya V. G., Schwab C. On singularities of solutions to the Dirichlet problem of hydrodynamics near the vertex of a cone // J. Reine Angew. Math. 1994. V. 456. P. 65-97.

[82] Kozlov V.A., Maz'ya V.G. Singularities in solutions to mathematical physics problems in nonsmooth domains // Partial Differential Equations and Functional Analysis. In memory of P. Grisvard. Proceedings of conference, 1994. Basel: Birkhäuser, 1996. P. 174-206.

[83] Pasy A. Asymptotic expansion of solution of ordinary differential equations in Hilbert space // Arch. Rational Mech. Anal. 1967. V. 24. P. 193-218.

[84] Назаров С. А. Весовые функции и инвариантные интегралы // Вычислительная механика деформируемого твердого тела. Т. 1, 1990. С. 17-31.

[85] Камоцкий И. В., Назаров С.А. Спектральные задачи в сингулярно возмущенных областях и самосопряженные расширения дифференциальных операторов // Труды СПбМО. 1998. Т. 6. С. 151-212.

[86] Назаров С. А. Трешина на стыке анизотропных тел. Сингулярности напряжений и инвариантные интегралы // Прикл. матем. мех. 1998. Т. 62. № 3. С. 489-502.

[87] Гохберг И. Ц., Крейн М. Г. Введение в теорию несамосопряженных операторов. М.: Наука, 1965.

[88] Михлин С. Г. Линейные уравнения в частных производных. М.: Высшая школа, 1977.

[89] Карпешина Ю. Е., Павлов Б. С. Взаимодействие нулевого радиуса для бигармонического и полигармонического уравнений // Матем. заметки. 1986. Т. 40. № 1. С. 49-59. 
[90] Березин Ф.А., Фаддеев Л. Д. Замечание об уравнении Шредингера с сингулярным потенциалом // Докл. АН СССР. 1961. Т. 137. № 5. С. 1011-1014.

[91] Павлов Б. С. Теория расширений и явнорешаемые модели // УМН. 1987. Т. 42. №6. C. $99-132$.

[92] Демков Ю.Н., Островский В.Н. Метод потенциалов нулевого радиуса в атомной физике. Л.: Изд-во ЛГУ, 1975.

[93] Канаун С. К., Левин В. М. Метод эффективного поля в механике композиционных материалов. Петрозаводск: Изд-во ПГУ, 1993.

[94] Назаров С. А. Эллиптические краевые задачи с периодическими коэффициентами в цилиндре // Изв. АН СССР. Сер. матем. 1981. Т. 45. №1. С. 101-112.

[95] Назаров С. А. О коэффициентах в асимптотике решений эллиптических краевых задач с периодическими коэффициентами // Вестник ЛГУ. 1985. №15. С. 16-22.

[96] Agmon S., Nirenberg L. Properties of solutions of ordinary differential equations in Banach space // Comm. Pure Appl. Math. 1963. V. 16. P. 121-239.

[97] Работнов Ю. Н. Механика деформируемого твердого тела. М.: Наука, 1979.

[98] Арутюнян Н.Х., Мовчан А.Б., Назаров С. А. Поведение решений задач теории упругости в неограниченных областях с параболоидальными и цилиндрическими включениями или полостями // Успехи механики. 1987. № 4. С. 3-91.

[99] Bayada C., Chambat M. The transition between the Stokes equations and the Reynolds equation: a mathematical proof // Appl. Math. Optim. 1986. V. 14. P. 73-93.

[100] Назаров С. А. Асимптотика решения задачи Навье-Стокса о течении тонкого слоя жидкости // Сиб. матем. журн. 1990. Т. 31. № 2. С. 131-144.

[101] Назаров С. А., Пилецкас К.И. Рейнольдсово течение жидкости в тонком трехмерном канале // Литов. матем. сб. 1990. Т. 30. № 4. С. 772-783.

[102] Тимошенко С. П., Войновский-Кригер С. Пластинки и оболочки. М.: Физматгиз, 1963.

[103] Амбарцумян С. А. Теория анизотропных пластин. М.: Наука, 1987.

[104] Зорин И. С. Операторное представление системы Ламе и предельные краевые задачи теории тонких плит // Вестник ЛГУ. 1987. №22. С. 108.

[105] Sanchez-Palencia E. Passage à la limite de l'élasticité tridimensionelle à la théorie asymptotique des coques minces // C. R. Acad. Sci. Paris. Sér. 2. 1990. V. 311. P. 909-916.

[106] Назаров С. А. Асимптотика решений эллиптической краевой задачи в тонкой области // Проблемы матем. анализа. Вып. 11. Л.: Изд-во ЛГУ, 1990. С. 191-208.

[107] Шойхет Б. А. Об асимптотически точных уравнениях тонких плит сложной структуры // Прикл. матем. мех. 1973. Т. 37. № 5. С. 913-924.

[108] Cioranescu D., Oleinik O. A., Tronel G. Korn's inequalities for frame structures and junctions with sharp estimates for the constants // Asymptotic Anal. 1994. V. 8. P. 1-14.

[109] Friedrichs K. O., Dressler R. F. A boundary-layer theory for elastic plates // Comm. Pure Appl. Math. 1961. V. 14. № 1. P. 1-33.

[110] Гольденвейзер А. Л. Теория упругих оболочек. М.: Наука, 1965.

[111] Gregery R. D., Wan F. V. Decaying states of plane strain in a semi-infinite strip and boundary conditions for plate theory // J. Elasticity. 1984. V. 14. № 1. P. 27-64.

[112] Зорин И. С., Назаров С. А.Краевой эффект при изгибе тонкой трехмерной пластины // Прикл. матем. мех. 1989. Т. 53. № 4. С. 642-650.

[113] Dauge M., Gruais I. Développement asymptotique d'ordre arbitraire pour une plaque élastique mince encatrée // C. R. Acad. Sci. Paris. Sér. 1. 1995. V. 321. P. 375-380.

[114] Dauge M., Djurdjevic I., Rössle A. Full asymptotic expansions for thin elastic free plates // C. R. Acad. Sci. Paris. Sér. 1. 1998. V. 326. P. 1243-1248.

[115] Ciarlet P. G. Plates junctions in elastic microstructures. Paris: Masson, 1990.

[116] Le Dret H. Problèmes variationnels dans les multi-domaines, modelisation des jonctions et applications. Paris: Masson, 1991.

[117] Panassenko G. P. Asymptotic analysis of bar systems. 1, 2 // Russian J. Math. Phys. 1994. V. 2. № 3. P. 325-353; 1996. V. 4. № 1. P. 37-116.

[118] Argatov I. I., Nazarov S. A. Junction problem of shashlik (skewer) type // C. R. Acad. Sci. Paris. Sér. 1. 1993. V. 316. P. 1329-1334. 
[119] Kozlov V.A., Maz'ya V.G., Movchan A.B. Asymptotic analysis of a mixed boundary value problem in a multi-structure // Asymptotic Anal. 1994. V. 8. P. 105-143.

[120] Назаров С. А. Соединения сингулярно вырождающихся областей различных предельных размерностей // Труды семин. им. И.Г. Петровского. Вып. 18. М. Изд-во МГУ, 1995. C. $3-78$.

[121] Nazarov S. A. Junction problems of bee-on-ceiling type in the theory of anisotropic elasticity // C. R. Acad. Sci. Paris. Sér. 1. 1995. V. 320. P. 1419-1424.

[122] Kozlov V.A., Maz'ya V. G., Movchan A. B. Asymptotic representation of elastic fields in a multi-structure // Asymptotic Anal. 1995. V. 11. P. 343-415.

[123] Аргатов И.И., Назаров С. А. Асимптотический анализ на соединениях областей различных предельных размерностей. Тело, пронзенное тонким стержнем // Изв. РАН. Сер. матем. 1996. Т. 60. №1. С. 3-36.

[124] Назаров С. А. Асимптотика решений задачи теории упругости для трехмерного тела с тонкими отростками // Докл. РАН. 1997. Т. 352. № 4. С. 458-461.

[125] Мельник Т. А., Назаров С.А. Асимптотика решения спектральной задачи Неймана в области типа "густого гребешка" // Труды семин. им. И.Г. Петровского. Вып. 19. М.: Изд-во МГУ, 1997. С. 138-173.

[126] Кучмент П.А. О теории Флоке для параболических и эллиптических граничных задач в цилиндре // Докл. АН СССР. 1981. Т. 258. № 2. С. 269-273.

[127] Кучмент П. А. Теория Флоке для дифференциальных уравнений в частных производных // УМH. 1982. T. 37. № 4. C. 3-52.

[128] Nicaise S., Sändig A.-M. General interface problems. 1, 2 // Math. Methods Appl. Sci. 1994. V. 17. P. 395-450.

Санкт-Петербургский государственный университет

Поступила в редакцию

E-mail: serna@snark.ipme.ru

15.04.1999 